March 16, 2018

\title{
Gravitational waves from plunges into Gargantua
}

\section{Geoffrey Compère ${ }^{\star 1}$, Kwinten Fransen*2, Thomas Hertog*3 and Jiang Long $\|^{\natural}$}

$\dagger$ Centre for Gravitational Waves, Université Libre de Bruxelles, International Solvay Institutes, CP 231, B-1050 Brussels, Belgium

* Centre for Gravitational Waves, Institute for Theoretical Physics, KU Leuven, Celestijnenlaan 200D, 3001 Leuven, Belgium

\begin{abstract}
We analytically compute time domain gravitational waveforms produced in the final stages of extreme mass ratio inspirals of non-spinning compact objects into supermassive nearly extremal Kerr black holes. Conformal symmetry relates all corotating equatorial orbits in the geodesic approximation to circular orbits through complex conformal transformations. We use this to obtain the time domain Teukolsky perturbations for generic equatorial corotating plunges in closed form. The resulting gravitational waveforms consist of an intermediate polynomial ringdown phase in which the decay rate depends on the impact parameters, followed by an exponential quasi-normal mode decay. The waveform amplitude exhibits critical behavior when the orbital angular momentum tends to a minimal value determined by the innermost stable circular orbit. We show that either near-critical or large angular momentum leads to a significant extension of the LISA observable volume of gravitational wave sources of this kind.
\end{abstract}

\footnotetext{
${ }^{1}$ email: gcompere@ulb.ac.be

${ }^{2}$ email: kwinten.fransen@kuleuven.be

${ }^{3}$ email: thomas.hertog@kuleuven.be

${ }^{4}$ email: jiang.long@ulb.ac.be
} 


\section{Contents}

\begin{tabular}{lll}
\hline 1 & Introduction & 2
\end{tabular}

2 The three extremal limits of Kerr 4

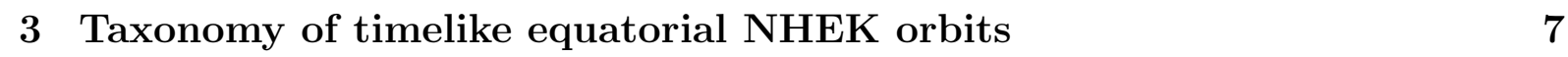

3.1 Timelike equatorial NHEK orbits . . . . . . . . . . . . . . . . . . 8

3.2 Timelike equatorial near-NHEK orbits . . . . . . . . . . . . . . . . . . . 9

3.3 Conformal transformations . . . . . . . . . . . . . . . . . . . . . . . . 9

\begin{tabular}{|lll}
\hline 4 & Extremal perturbation theory & 13
\end{tabular}

4.1 Gravitational perturbations of Kerr . . . . . . . . . . . . . . . . . . . . 13

4.2 Quasi-normal mode approximation . . . . . . . . . . . . . . . . . . 19

$\begin{array}{lll}5 & \text { Emission from circular near-NHEK orbits } & 21\end{array}$

$6 \quad$ Emission from generic orbits from conformal transformations 25

6.1 Circular near-NHEK orbit to NHEK orbits . . . . . . . . . . . . . . 26

6.2 Circular NHEK orbit to NHEK orbits . . . . . . . . . . . . . . . . . . . . . 34

6.3 Circular NHEK orbit to near-NHEK orbits . . . . . . . . . . . . . . . . . 35

6.4 Circular near-NHEK orbit to near-NHEK orbits . . . . . . . . . . . . . . . 39

6.5 Summary and comparison . . . . . . . . . . . . . . . . 40

\begin{tabular}{lll}
\hline 7 & Critical behavior & 42
\end{tabular}

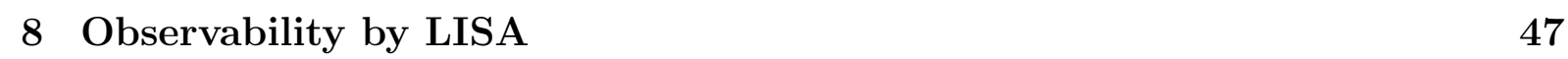

$\begin{array}{lll}9 & \text { Summary } & 50\end{array}$

\begin{tabular}{|l}
\hline A Teukolsky's formalism \\
\hline
\end{tabular}

A.1 Newman-Penrose tetrad . . . . . . . . . . . . . . . . . . . . . 52

A.2 Master equation of Teukolsky . . . . . . . . . . . . . . . . . . . . 55

A.3 Separation of variables . . . . . . . . . . . . . . . . . . . 57

B Detailed taxonomy of (near-)NHEK orbits 60

B.1 Timelike NHEK orbits . . . . . . . . . . . . . . . . . . . . . . . 60

B.2 Timelike near-NHEK orbits . . . . . . . . . . . . . . . . . 61

B.3 Conformal transformations . . . . . . . . . . . . . . . . . . . . 62

\begin{tabular}{lll}
\hline C Emission from the ISCO orbit in NHEK & 67
\end{tabular}

\begin{tabular}{|ll|}
\hline D Relevant integrals and QNM overtone summation & 70
\end{tabular} 


\section{Introduction}

Extreme Mass Ratio Inspirals (EMRIs) describe the long-lasting inspiral - from months to a few years - and final plunge of stellar mass black holes into supermassive black holes in the centre of galaxies. The gravitational wave (GW) pattern emitted by EMRIs encodes detailed and rich information about the strong gravity region in the neighbourhood of the central massive object. The observation of EMRIs is therefore one of the central goals of LISA, the planned space-based gravitational wave observatory [1-3].

To fully exploit the scientific potential of EMRI observations requires an extremely accurate modelling of the relativistic dynamics over a large number of cycles. However the construction of precise gravitational waveform templates needed to interpret LISA observations of EMRIs is a challenge at several levels. First, the orbits are highly relativistic which makes the post-Newtonian methods commonly used in the LIGO/VIRGO data analysis inadequate. Second, the large number of observable cycles and the separation of scales pose severe difficulties for numerical simulations. Finally, self-force effects which are relevant corrections in perturbation methods still require further development [4 -10$]$.

In this paper we identify new corners in the parameter space of EMRIs where semianalytical waveforms can be computed from first principles. Specifically we consider EMRIs involving a nearly maximally spinning supermassive black hole, which we refer to as Gargantua, following the terminology of $\left[\left.11\right|^{5}\right.$ The comparison between the analytic leading order near-horizon results and numerical results for gravitational emission on the ISCO orbit allows to test the precision of the leading order near-horizon methods. For $1-J / M^{2} \sim 10^{-4}$ one obtains $\sim 10 \%$ precision, and $1-J / M^{2} \sim 10^{-6}$ gives $\sim 1 \%$ precision ${ }^{6}$. According to recent X-ray observing campaigns, 7 out of the 22 Active Galactic Nuclei (AGNs) analyzed are candidates for being high spin supermassive black holes 7 . Accretion is the main mechanism to spin up black holes. For geometrically thin disk models this leads to the Thorne bound $J / M^{2}<99.8 \%[18]$. Spins near the upper bound are reached according to the black hole spin evolution model of [19] for supermassive black holes of $10^{9}$ solar masses or higher, if the accretion gas is anisotropic. The Thorne bound can however be exceeded in the presence of magnetic fields, as shown e.g. in the magnetohydrodynamic simulations of [20]. The number of EMRI events expected to be detected by LISA ranges from a few to a few thousands per year due to astrophysical uncertainties [3]. The currently preferred population model for LISA uses a Gaussian distribution of spins centered at $98 \%$ and capped off at the Thorne bound [3]. However in light of [20] EMRIs

\footnotetext{
${ }^{5}$ Gargantua refers to the black hole featured in Nolan's science-fiction movie Interstellar, which according to Thorne $\left[12\right.$ must be very rapidly spinning, with $1-J / M^{2}<10^{-14}$ to allow key pieces of the narrative.

${ }^{6}$ See Table I of [13]. For the precision of near-horizon methods in the context of accretion disks, see 14 .

${ }^{7}$ See Table 1 of 15]. These are MCG-6-30-15 16], 1H 0707-495 [17, NGC 3783, Mrk 110, RBS 1124, IRAS 13224-3809 and NGC 4051.
} 
involving a high spin supermassive black holes with $J / M^{2}=0.9999$ are candidate events for the LISA observatory.

The physics of such black holes in the near-horizon region is controlled by $S L(2, \mathbb{R})$ conformal symmetry 21 24 and, at the quantum level, by infinite-dimensional conformal symmetries 2527$]$. The combination of a small near-extremality parameter and near-horizon $S L(2, \mathbb{R})$ conformal symmetry allows one to use unique analytical methods, including matched asymptotic expansions and conformal representation theory methods, which in turn enables one to obtain gravitational waveforms in closed form. This program was initiated in 13,28 31]. Here we extend this to generic corotating equatorial orbits into supermassive, nearly extremal Kerr black holes. All known previous results for gravitational wave emission [13, 28, 29] are particular instances of our general analysis, which also generalizes to spin 2 the known analogous spin 0 results [30,31. Moreover, the semi-analytic waveforms we obtain contain novel remarkable "smoking gun" signatures associated with near extremality. They should also be useful to calibrate numerical simulations and effective models, and serve as a basis for further analytical endeavor.

The opening up of an extended near-horizon region at high redshift around rapidly spinning black holes gives rise to very specific strong gravity physics. For instance the Aretakis instability occurs in the extremal limit [32] and leads to the amplification of signals emerging from the near-horizon due to high-energy interaction with near-horizon modes 33 35]. In [11] it was found that the EMRI GW signal obtained by adiabatically evolving the ISCO into Gargantua develops a characteristic tail associated with GW emission during the late, near-horizon phase of the inspiral. Here we obtain new gravitational wave signatures specific to plunges of non-spinning compact objects along general corotating geodesic orbits into Gargantua. Importantly, the spectrum of quasi-normal modes splits in the near-extremal limit into damped modes in the asymptotically flat region, and zero-damped modes in the near-horizon region [36]. This leads to polynomial quasinormal mode ringing due to harmonic stacking of overtones [37]. Polynomial ringing was described so far without reference to the source of gravitational waves. Here we show that this effect is a manifest feature or a "smoking gun" signature of the gravitational wave emission from a plunging source into Gargantua. We also discuss the implications and the observability of the resulting signal for LISA.

As mentioned already, a distinctive feature of EMRIs into Gargantua is the presence of spontaneously broken conformal symmetry. The existence of the asymptotic extremal system with exact conformal symmetry leads to critical behavior, as already demonstrated for various physical processes probing nearly maximally spinning black holes [14, 38, 39]. Here we find a new kind of critical behavior, directly associated with the GW emission from EMRIs, which occurs when the orbital angular momentum of the plunging body approaches a minimal angular momentum bound set by the innermost stable circular orbit. We find that in this critical limit, the amplitude of the GW signal in the geodesic approximation diverges. Hence one expects that non-linear backreaction becomes impor- 
tant. On a more technical point we note that the existence of conformal symmetry is the key feature that allows us to explicitly evaluate the Fourier integrals and to resum the zero-damped quasi-normal modes which ultimately leads to the closed form, analytical, late time domain waveform for all equatorial plunging orbits.

The paper is organized as follows. In Section 2, we recall the three distinct spacetime regions that arise from the Kerr metric in the near-extremal limit. In Section 3 we perform a systematic classification of conjugacy classes of equatorial timelike geodesics under complexified conformal symmetry, which consists of $S L(2, \mathbb{C}) \times U(1)$ transformations combined with PT symmetry (flip of time and axial angle). We will derive that there are only two conjugacy classes whose representatives can be chosen to be the circular orbit in either NHEK or near-NHEK. All other trajectories can be obtained from conformal symmetry by employing complex transformations. In Section 4 we obtain the general solution (up to an important coefficient that depends on the source) of the Teukolsky master equation governing gravitational perturbations of nearly-extremal Kerr, using the method of matched asymptotic expansions and with appropriate boundary conditions. Selecting the zero-damped QNM contribution we also convert the frequency-based solution for the perturbation into a waveform in the time domain at late times. In Section 5 we obtain the spectrum of GW emission of a body moving on a circular geodesic in (near)NHEK at first order in the asymptotically matched expansion. We use this in Section 6 to derive the gravitational waveforms for all other equatorial (corotating) orbits in (near-)NHEK by applying the conformal transformations of Section 3 which relate the waveforms associated with generic equatorial plunges in (near-)NHEK to one of the two sets of circular "seed orbits". We obtain the analytical critical behavior of all resulting waveforms in Section 7 in the limit of minimal angular momentum compatible with the existence of (near-)NHEK orbits. We also obtain the analytical large orbital angular momentum behavior. We discuss the observability of such signals by LISA in Section 8 . We conclude in Section 9 ,

\section{The three extremal limits of Kerr}

Given a metric depending on a parameter $\lambda$, the limiting spacetime when $\lambda \rightarrow 0$ might depend on the coordinates in which the limit was taken [40]. For a near-extremal Kerr black hole with

$$
\lambda \equiv \sqrt{1-\frac{J^{2}}{M^{4}}} \ll 1,
$$

it turns out that there exist 3 distinct coordinate systems that admit a distinct spacetime limit when $\lambda \rightarrow 0$, as we will now review. In turn, this implies that a near-extremal Kerr black hole is composed of three patches glued together that each resolve the physics in a 
distinct scaling limit: the extremal Kerr region, the near-horizon extremal Kerr (NHEK) region and the very near-horizon region (near-NHEK).

The extremal Kerr metric is obtained by taking the limit $\lambda \rightarrow 0$ in Boyer-Linquist coordinates $(\hat{t}, \hat{r}, \theta, \hat{\phi})$. We recall that the Kerr metric in Boyer-Linquist coordinates is given by

$$
\begin{aligned}
d s^{2}= & -\left(1-\frac{2 M \hat{r}}{\Sigma}\right) d \hat{t}^{2}+\frac{\Sigma}{\Delta} d \hat{r}^{2}+\Sigma d \theta^{2}+\left(\hat{r}^{2}+a^{2}+\frac{2 M a^{2} \hat{r} \sin ^{2} \theta}{\Sigma}\right) \sin ^{2} \theta d \hat{\phi}^{2} \\
& -\frac{4 M a \hat{r} \sin ^{2} \theta}{\Sigma} d \hat{t} d \hat{\phi}
\end{aligned}
$$

where

$$
\Delta \equiv \hat{r}^{2}-2 M \hat{r}+a^{2}, \quad \Sigma \equiv \hat{r}^{2}+a^{2} \cos ^{2} \theta .
$$

The inner and outer horizons are denoted as $\hat{r}_{ \pm}=M \pm \sqrt{M^{2}-a^{2}}$ or $\hat{r}_{ \pm}=M(1 \pm \lambda)$. The angular velocity and Hawking temperature are denoted as $\Omega_{H}=a /\left(2 M r_{+}\right)$and $T_{H}=\left(r_{+}-M\right) /\left(4 \pi M r_{+}\right)$. The radial tortoise coordinate $\hat{r}^{*}$ of the Kerr metric is defined as

$$
\hat{r}^{*}=\hat{r}+\frac{2 M \hat{r}_{+}}{\hat{r}_{+}-\hat{r}_{-}} \ln \frac{\hat{r}-\hat{r}_{+}}{2 M}-\frac{2 M \hat{r}_{-}}{\hat{r}_{+}-\hat{r}_{-}} \ln \frac{\hat{r}-\hat{r}_{-}}{2 M} .
$$

The limit $\lambda \rightarrow 0$ in these coordinates gives the extremal Kerr metric.

It was first observed by Bardeen, Press and Teukolsky [41] that in the extremal limit the innermost stable circular orbit (ISCO) and the innermost bound circular orbit (IBCO) (sometimes called the marginally bound circular orbit) admit distinct scaling limits: $\hat{r}_{I S C O}=M+2^{1 / 3} \lambda^{2 / 3} M+O(\lambda)$ while $\hat{r}_{I B C O}=M+2^{1 / 2} \lambda M+o(\lambda)$. These timelike orbits degenerate with the null event horizon in Boyer-Linquist coordinates. Therefore, two additional separate coordinate systems are required to resolve the physics around these orbits, which turn out to lead to distinct limiting spacetimes.

The near-horizon extremal Kerr geometry (NHEK) 21]

$$
d s^{2}=2 M^{2} \Gamma(\theta)\left(-R^{2} d T^{2}+\frac{d R^{2}}{R^{2}}+d \theta^{2}+\Lambda^{2}(\theta)(d \Phi+R d T)^{2}\right)
$$

where

$$
\Gamma(\theta)=\frac{1+\cos ^{2} \theta}{2}, \quad \Lambda(\theta)=\frac{2 \sin \theta}{1+\cos ^{2} \theta}
$$

is obtained from the Kerr geometry in the limit $\lambda \rightarrow 0$ after a zoom close to the horizon in corotating frame,

$$
\begin{aligned}
T & =\frac{\hat{t}}{2 M} \lambda^{2 / 3} \\
R & =\frac{\hat{r}-\hat{r}_{+}}{M} \lambda^{-2 / 3}, \\
\Phi & =\hat{\phi}-\Omega_{e x t} \hat{t}, \quad \Omega_{e x t} \equiv \frac{1}{2 M} .
\end{aligned}
$$


We call the coordinates $(T, R, \theta, \Phi)$ the Poincaré coordinates of NHEK. The event horizon is mapped in the limit to the NHEK Poincaré horizon $R=0$. This spacetime admits an enhanced $S L(2, \mathbb{R}) \times U(1)$ Killing symmetry. By construction, the ISCO is mapped into the NHEK orbit $R=2^{1 / 3}$ in the near-extremal limit but because of the emerging scale invariance, the orbit $R=2^{1 / 3}$ takes no specific role within the NHEK spacetime alone. The location $R=2^{1 / 3}$ takes the meaning to be the ISCO only after specifying how the asymptotically flat region is glued to the NHEK region through the specific coordinate change (2.7). This spacetime is relevant for Boyer-Linquist coordinates in the range $\hat{r} \sim \hat{r}_{+}+M \times O\left(\lambda^{2 / 3}\right)$.

The very near-horizon extremal Kerr geometry (near-NHEK) 22, 24]

$$
d s^{2}=2 M^{2} \Gamma(\theta)\left(-r(r+2 \kappa) d t^{2}+\frac{d r^{2}}{r(r+2 \kappa)}+d \theta^{2}+\Lambda^{2}(\theta)(d \phi+(r+\kappa) d t)^{2}\right)
$$

is obtained from the Kerr geometry in the limit $\lambda \rightarrow 0$ via the change of coordinates

$$
\begin{aligned}
t & =\frac{\hat{t}}{2 M \kappa} \lambda, \\
r & =\kappa \frac{\hat{r}-\hat{r}_{+}}{M \lambda}, \\
\phi & =\hat{\phi}-\frac{\hat{t}}{2 M},
\end{aligned}
$$

where $\kappa>0$ is arbitrary as a consequence of emerging scale invariance. The event horizon is mapped in the limit to the NHEK black hole horizon $r=0$. By definition, this spacetime is relevant for Boyer-Linquist coordinates in the range $\hat{r} \sim \hat{r}_{+}+M \times O(\lambda)$ which is closer to the horizon than the NHEK patch.

The NHEK and near-NHEK spacetimes are diffeomorphic to each other. The nearNHEK coordinate patch $(t, r, \phi)$ associated with the parameter $\kappa$ that lies at the lowest corner of the NHEK Poincaré patch $(T, R, \Phi)$, is given by

$$
\begin{aligned}
R & =\frac{1}{\kappa} e^{\kappa t} \sqrt{r(r+2 \kappa)}, \\
T & =-e^{-\kappa t} \frac{r+\kappa}{\sqrt{r(r+2 \kappa)}}, \\
\Phi & =\phi-\frac{1}{2} \log \frac{r}{r+2 \kappa} .
\end{aligned}
$$

While the global patch of NHEK does not appear as a limit of the Kerr metric, it will turn out to be useful in order to map the NHEK geodesic orbits and draw their Penrose

${ }^{8}$ In fact $\hat{r} \sim \hat{r}_{+}+M \times O\left(\lambda^{p}\right)$ for $0<p<1$ but the ISCO limit is defined only for $p=\frac{2}{3}$. 
diagrams. We use the standard embedding of the Poincaré coordinate patch $(T, R, \Phi)$ in the global coordinate patch $(\tau, y, \varphi)$ of the NHEK spacetime given by

$$
\begin{aligned}
R & =\sqrt{1+y^{2}} \cos \tau+y \\
T & =\sqrt{1+y^{2}} \sin \tau \frac{1}{R} \\
\Phi & =\varphi+\log \frac{\cos \tau+y \sin \tau}{1+\sqrt{1+y^{2}} \sin \tau}
\end{aligned}
$$

where the global metric $(-\infty<\tau<\infty,-\infty<y<\infty)$ is

$$
d s^{2}=2 M^{2} \Gamma(\theta)\left(-\left(1+y^{2}\right) d \tau^{2}+\frac{d y^{2}}{1+y^{2}}+d \theta^{2}+\Lambda^{2}(\theta)(d \phi+y d \tau)^{2}\right) .
$$

Given the $2 \pi$-periodicity of $\tau$, the conformal Penrose diagram can be drawn as a rectangle

\begin{tabular}{|c|c|c|c|c|}
\hline Coordinates & & Metric & Coordinate changes & \\
\hline \multirow[t]{2}{*}{ Boyer-Linquist } & $(\hat{t}, \hat{r}, \theta, \hat{\phi})$ & 2.2 & $(\hat{t}, \hat{r}, \theta, \hat{\phi}) \rightarrow(T, R, \theta, \Phi)$ & 2.7 \\
\hline & & & $(\hat{t}, \hat{r}, \theta, \hat{\phi}) \rightarrow(t, r, \theta, \phi)$ & 2.9 \\
\hline \multirow[t]{2}{*}{ Poincaré NHEK } & $(T, R, \theta, \Phi)$ & 2.5 & $(T, R, \theta, \Phi) \rightarrow(t, r, \theta, \phi)$ & 2.10 \\
\hline & & & $(T, R, \theta, \Phi) \rightarrow(\tau, y, \theta, \phi)$ & 2.11 \\
\hline near-NHEK & $(t, r, \theta, \phi)$ & 2.8 & $(t, r, \theta, \phi) \rightarrow(T, R, \theta, \Phi)$ & B.20 \\
\hline global NHEK & $(\tau, y, \theta, \phi)$ & 2.12 & $(\tau, y, \theta, \phi) \rightarrow(T, R, \theta, \Phi)$ & B.18 \\
\hline
\end{tabular}
$-\pi<\tau<\pi,-\frac{\pi}{2}<\arctan (y)<\frac{\pi}{2}$ where each point represents a two-sphere. For the convenience of the reader, we summarized our notation for the different coordinate systems and their relationship in Table 1.

Table 1: Summary of the different coordinates and their relations.

\section{Taxonomy of timelike equatorial NHEK orbits}

The generic trajectory of a massive probe such as a star or a stellar black hole falling into a nearly extreme, spinning, supermassive black hole can be approximated in its vicinity by a null geodesic in one of the near-horizon geometries. This is due to the infinite redshift in the extremal limit which requires a rescaling of proper time. In the special case where the probe is nearly co-rotating with the central black hole, the trajectory can instead be approximated by a timelike geodesic in one of the near-horizon geometries. 
All previous studies of gravitational wave emission in the near-horizon Kerr geometry have concentrated on specific equatorial timelike geodesics and specific $S L(2, \mathbb{R})$ transformations relating them [11, 13, 28 31]. Here, we go further and perform a systematic classification of conjugacy classes of equatorial timelike geodesics under conformal symmetry, which consists of complexified $S L(2, \mathbb{C}) \times U(1)$ transformations combined with PT symmetry (flip of time and axial angle). We will see there are only two conjugacy classes whose representatives can be chosen to be the circular orbit in NHEK and the circular orbit in near-NHEK. All other (real) trajectories can be obtained from conformal symmetry by employing complex transformations. It therefore suffices to understand the physics of circular orbits in (near-)NHEK to understand all equatorial timelike orbits.

The energy per unit probe mass $\hat{E}$ associated with the asymptotically flat Killing vector $-\partial_{\hat{t}}$ is conserved and positive. By contrast there is no global timelike Killing vector associated with the (near-)NHEK regions. In particular $\partial_{T}, \partial_{t}$ are spacelike, so the NHEK energy $E$ associated with $-\partial_{T}$ and the near-NHEK energy $e$ associated with $-\partial_{t}$ can be negative. We denote by $\ell$ the angular momentum per unit probe mass associated with $\partial_{\hat{\phi}}, \partial_{\Phi}$, and $\partial_{\phi}$. We will often call the quantity $\ell$ angular momentum in what follows by a slight abuse of language. These quantities are related as

$$
\hat{E}=\Omega_{E x t} \ell+\frac{\lambda^{2 / 3}}{2 M} E=\Omega_{E x t} \ell+\frac{\lambda}{2} \frac{e}{M \kappa}
$$

where $\Omega_{E x t}=1 /(2 M)$ and $\lambda$ is the near-extremal redshift factor (2.1).

\subsection{Timelike equatorial NHEK orbits}

We are interested in future-directed timelike orbits entering the NHEK geometry (which is equivalent to near-NHEK with $\kappa=0$ ) from the asymptotically flat region. Accordingly we consider $d T / d R<0$ at $R \rightarrow \infty$. We only look at orbits corotating with the black hole $(\ell \geq 0)$. It turns out there are no orbits for which the conserved angular momentum per unit probe mass $\ell$ is lower than the critical angular momentum $\ell_{*}$, with

$$
\ell_{*}=\frac{2}{\sqrt{3}} M .
$$

We distinguish critical $\ell=\ell^{*}$ from supercritical $\ell>\ell^{*}$ orbits. Plunges are defined as orbits that cross the horizon in finite affine time. Osculating orbits are defined as orbits that leave the NHEK geometry in finite affine time. In the marginal case, the orbit leaves the NHEK geometry at infinite time $T$. We find the following distinct orbits (where critical orbits are indicated with a $*$ subscript): 


$$
\begin{array}{llll}
\kappa=0 & \ell=\ell_{*} & E<0 & \text { Does not exist } \\
\kappa=0 & \ell=\ell_{*} & E=0 & \operatorname{Circular}_{*}(\text { ISCO) } \\
\kappa=0 & \ell=\ell_{*} & E>0 & \operatorname{Plunging}_{*}(E) \\
\kappa=0 & \ell>\ell_{*} & E<0 & \operatorname{Osculating}(E, \ell) \\
\kappa=0 & \ell>\ell_{*} & E=0 & \operatorname{Marginal}(\ell) \\
\kappa=0 & \ell>\ell_{*} & E>0 & \operatorname{Plunging}(E, \ell)
\end{array}
$$

All such orbits are detailed in Appendix B.1 and illustrated in Figure 1. The Plunging ${ }_{*}(E)$ orbit is also known as the fast NHEK plunge [30].

\subsection{Timelike equatorial near-NHEK orbits}

After analysis, we obtain the following orbits:

$$
\begin{array}{llll}
\kappa>0 & \ell=\ell_{*} & e<0 & \text { Does not exist } \\
\kappa>0 & \ell=\ell_{*} & e=0 & \operatorname{Plunging}_{*}(e=0) \\
\kappa>0 & \ell=\ell_{*} & 0<e<\kappa \ell^{*} & \operatorname{Plunging}_{*}(e) \\
\kappa>0 & \ell=\ell_{*} & e=\kappa \ell^{*} & \operatorname{Plunging}_{*}\left(e=\kappa \ell^{*}\right) \\
\kappa>0 & \ell=\ell_{*} & e>\kappa \ell^{*} & \operatorname{Plunging}_{*}(e) \\
\kappa>0 & \ell>\ell_{*} & e<-\frac{\kappa}{2} \sqrt{3\left(\ell^{2}-\ell_{*}^{2}\right)} & \operatorname{Osculating}(e, \ell) \\
\kappa>0 & \ell>\ell_{*} & e=-\frac{\kappa}{2} \sqrt{3\left(\ell^{2}-\ell_{*}^{2}\right)} & \operatorname{Circular}(\ell) \\
\kappa>0 & \ell>\ell_{*} & e>-\frac{\kappa}{2} \sqrt{3\left(\ell^{2}-\ell_{*}^{2}\right)} & \operatorname{Plunging}(e, \ell)
\end{array}
$$

In addition for the specific initial condition $t_{0} \rightarrow-\infty$ there is another family of circular orbits specified by

$$
\kappa>0 \quad \ell>\ell_{*} \quad e=-\kappa \ell \quad \text { Special } \operatorname{Circular}(\ell) .
$$

All orbits are detailed in Appendix B.2. The Plunging ${ }_{*}(e=0)$ orbit was studied in $[29]$. The Plunging ${ }_{*}(e)$ orbits are also known as the near-NHEK fast plunges [30]. The Osculating $(e, \ell)$ and Plunging $(e, \ell)$ orbits were described in [31].

We illustrate these orbits in Figures 2 and 3 where we show the conformal diagram of the global NHEK geometry from $\tau=-\frac{\pi}{2}$ to $\tau=+\frac{\pi}{2}$, with the Poincaré and near-NHEK horizons indicated in blue. We used $M=\kappa=1$ and when depicting $\ell>\ell^{*}$ orbits, we chose $\ell=2 \ell^{*}$. The Plunging $(e)$ orbits with $e$ smaller or bigger than $\kappa \ell^{*}$ are distinguished by their behavior inside the near-NHEK horizon.

\subsection{Conformal transformations}

Let us now characterize how conformal symmetry or more precisely $S L(2, \mathbb{R}) \times U(1)$ symmetry can be used to relate various equatorial orbits. Since $U(1)$ symmetry commutes 


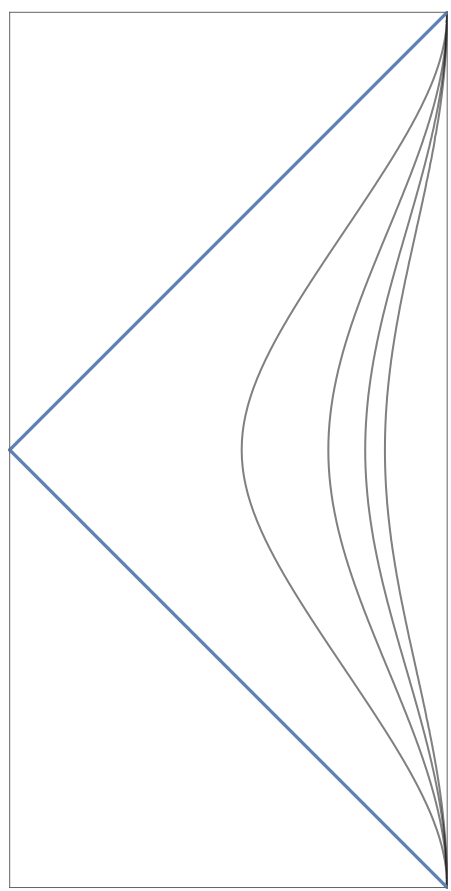

(a) Circular $_{*}$ (ISCO)

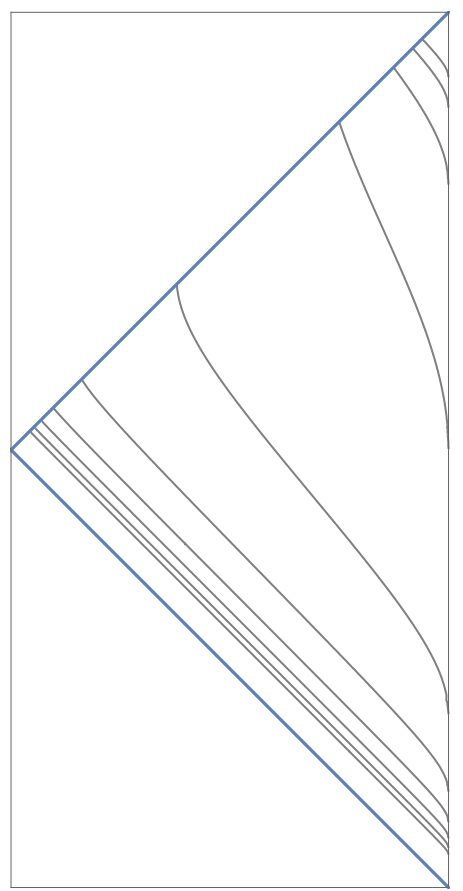

(b) Plunging $_{*}(E)$

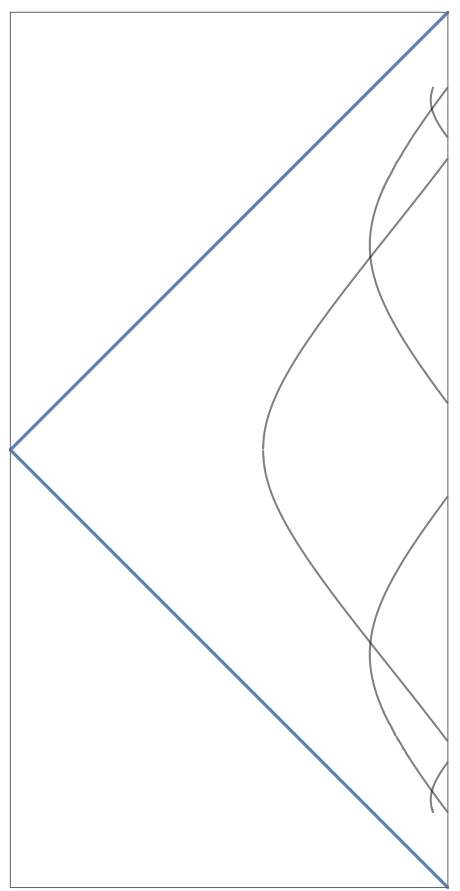

(c) Osculating $(E, \ell)$

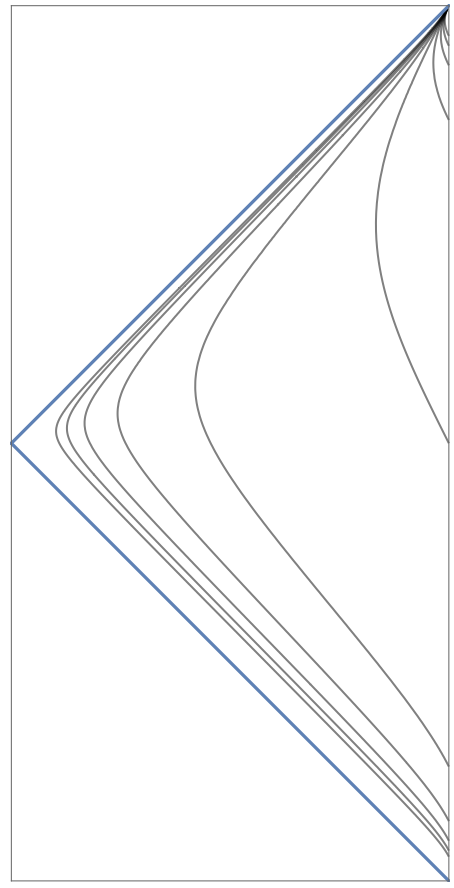

(d) Marginal $(\ell)$

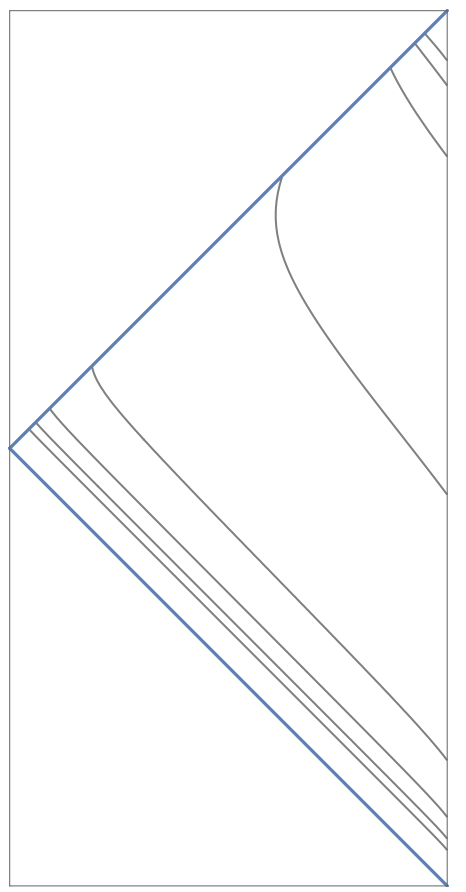

(e) Plunging $(E, \ell)$

Figure 1: Taxonomy of incoming, timelike NHEK orbits shown here in the conformal diagram of global NHEK. The vertical axis ranges from $\tau=-\pi$ to $\tau=+\pi$ and the horizontal axis from $\arctan (y)=-\frac{\pi}{2}$ to $+\frac{\pi}{2}$, see Section 2 for definitions. The blue lines at $45^{\circ}$ and $135^{\circ}$ indicate the Poincaré horizon. In particular, the blue line at $45^{\circ}$ is the event horizon. We have used $M=1$ and, for $\ell>\ell^{*}$ orbits, we have taken $\ell=2 \ell^{*}$. 


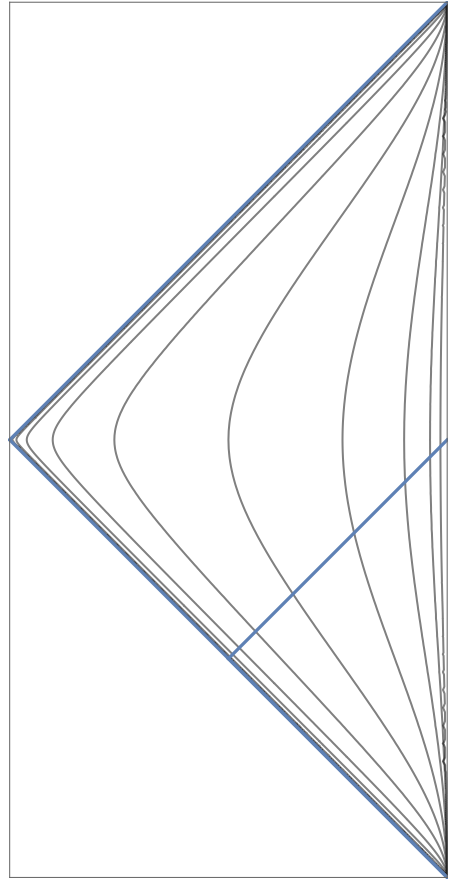

(a) Plunging $_{*}(e=0)$

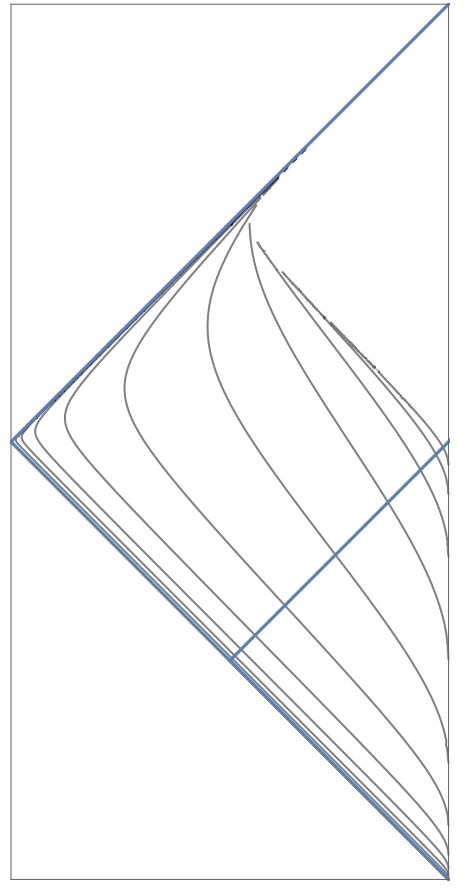

(b) Plunging $_{*}\left(0<e<\kappa \ell^{*}\right)$

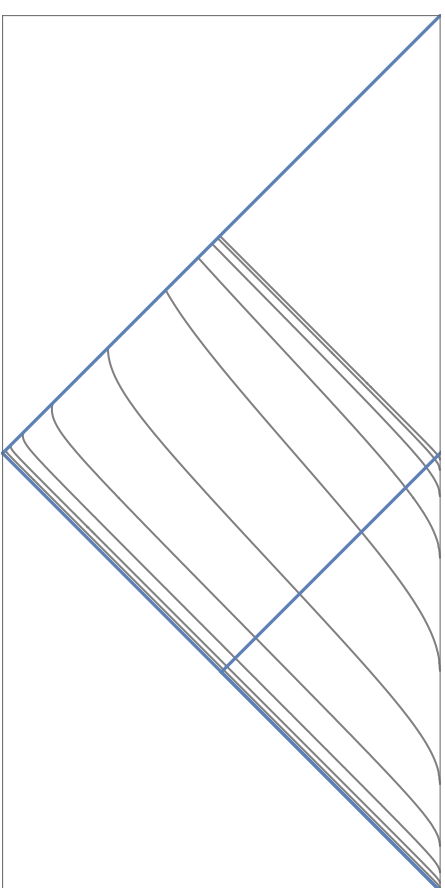

(d) Plunging $_{*}\left(e>\kappa \ell^{*}\right)$

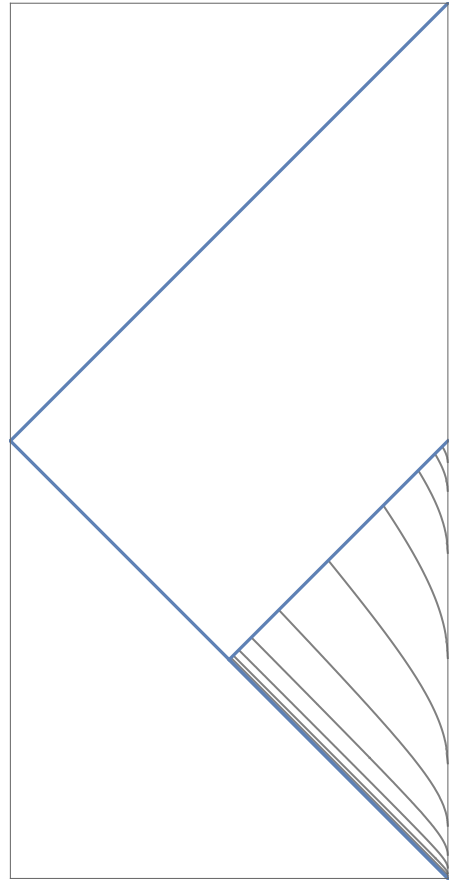

(c) $\operatorname{Plunging}_{*}\left(e=\kappa \ell^{*}\right)$

Figure 2: Taxonomy of incoming timelike near-NHEK orbits (critical orbits). The axis and choice of parameters are defined as in Figure 1. The outermost blue lines at $45^{\circ}$ and $135^{\circ}$ indicate the Poincaré horizon $R=0$ of the embedding Poincaré NHEK patch. The innermost blue lines at $45^{\circ}$ and $135^{\circ}$ (degenerate with the Poincaré line) indicate the NHEK black hole horizon $r=0$. In particular, the innermost blue line at $45^{\circ}$ is the event horizon. We draw the orbits past the event horizon in order to visualize the real conformal maps of orbits (the complex ones do not allow such a visualization). 


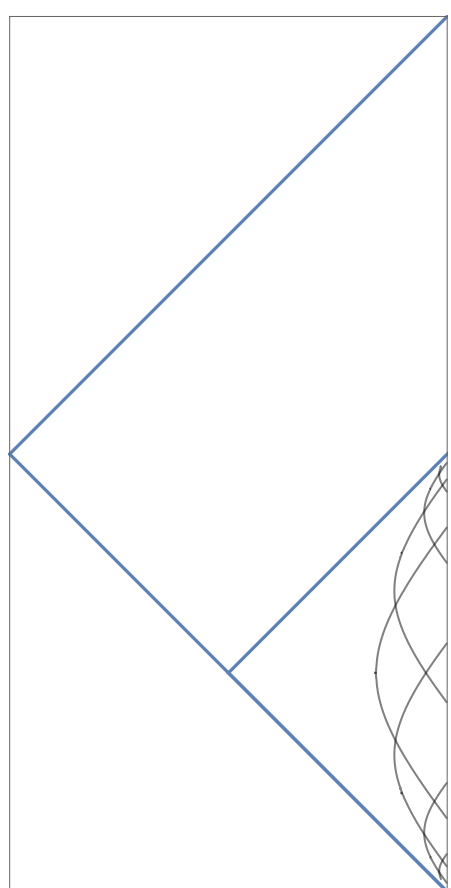

(a) Osculating $(e, \ell) \quad(e<$ $\left.-\frac{\kappa}{2} \sqrt{3\left(\ell^{2}-\ell_{*}^{2}\right)}\right)$

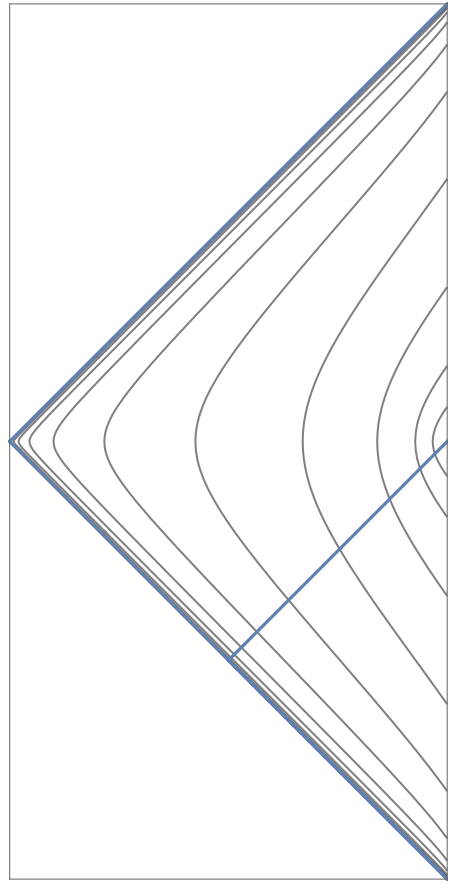

(d) Plunging $(e, \ell)(e=0)$

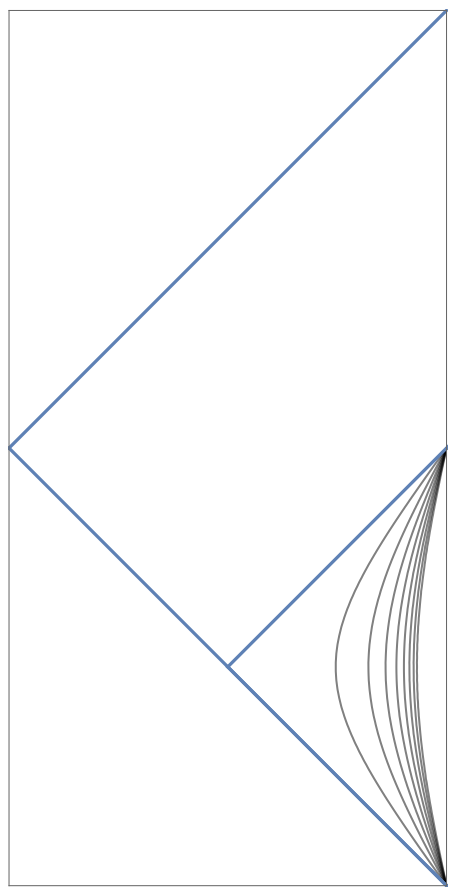

(b) $\left(e=-\frac{\kappa}{2} \sqrt{3\left(\ell^{2}-\ell_{*}^{2}\right)}\right)$ or Second Circular $(\ell)(e=-\kappa \ell)$

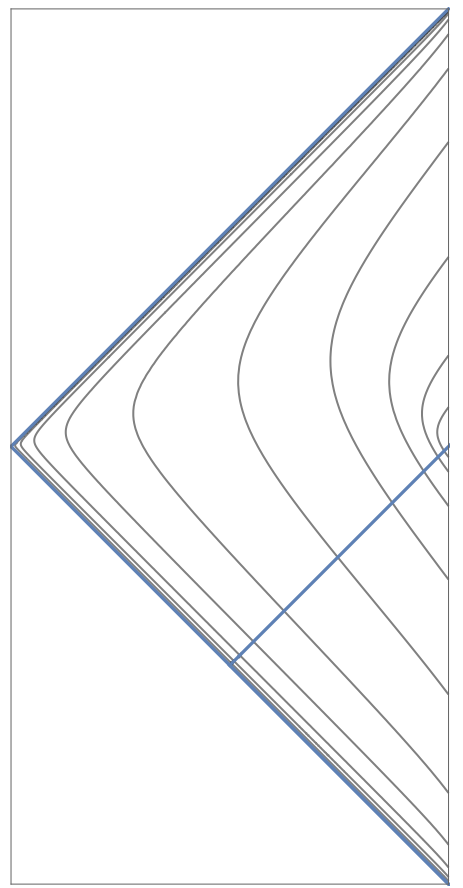

(e) Plunging $(e, \ell)$ with $0<$ $e<\kappa \ell^{*}$

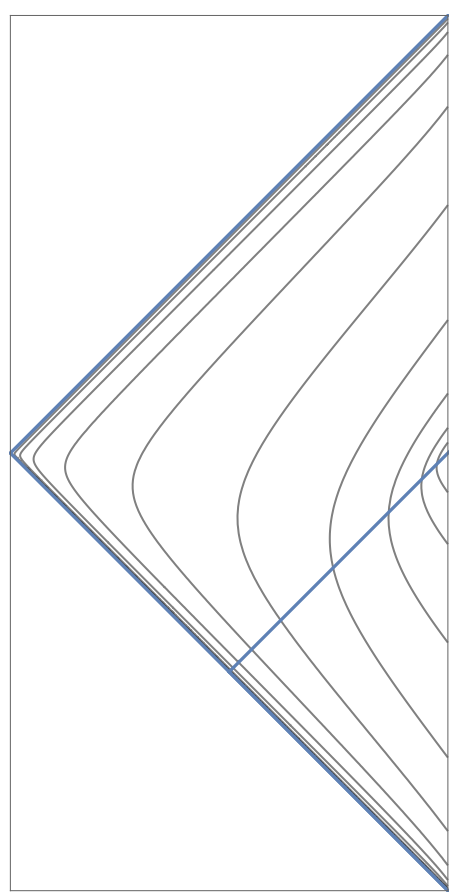

(c) Plunging $(e, \ell)$ $\left(-\frac{\kappa}{2} \sqrt{3\left(\ell^{2}-\ell_{*}^{2}\right)}<e<0\right)$

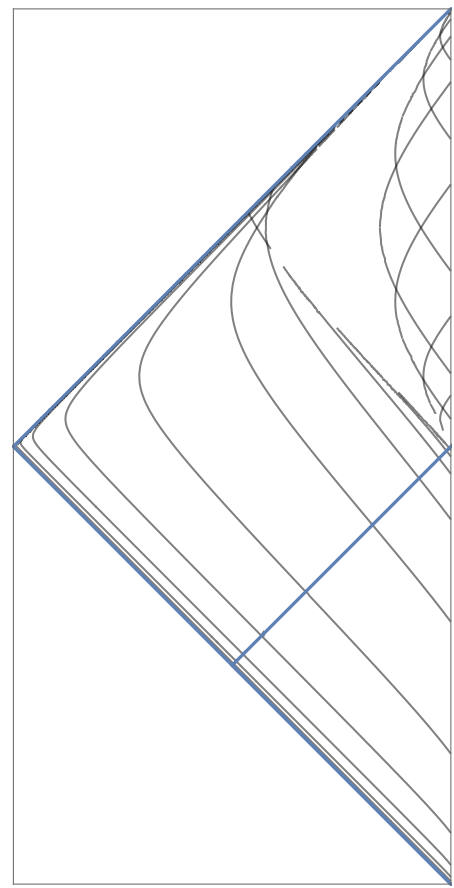

(f) Plunging $(e, \ell)$ with $e \geq$ $\kappa \ell^{*}$

Figure 3: Taxonomy of incoming timelike near-NHEK orbits (supercritical orbits). (The broken lines in $(f)$ are unphysical numerical rendering effects) 
with all symmetries, the angular momentum is invariant under symmetry transformations. One can therefore only attempt to map the $\ell=\ell^{*}$ orbit to another $\ell=\ell^{*}$ orbit, and a $\ell>\ell^{*}$ orbit to another $\ell>\ell^{*}$ orbit.

After analysis, it turns out that all equatorial orbits can be related to either a NHEK circular orbit or near-NHEK circular orbit by a complex $S L(2, \mathbb{C}) \times U(1)$ transformation which could be combined with a PT symmetry flip (time and axial angle). There exist therefore exactly two conjugacy classes of orbits under $S L(2, \mathbb{C}) \times U(1) \times P T$ symmetry. These are

- $\operatorname{Circular}_{*}(\mathrm{ISCO}) \Leftrightarrow \operatorname{Plunging}_{*}(E) \Leftrightarrow \operatorname{Plunging}_{*}(e=0) \Leftrightarrow \operatorname{Plunging}_{*}(e)$

- $\operatorname{Circular}(\ell) \Leftrightarrow \operatorname{Marginal}(\ell) \Leftrightarrow \operatorname{Osculating}(E, \ell) \Leftrightarrow \operatorname{Plunging}(E, \ell) \Leftrightarrow \operatorname{Osculating}(e, \ell)$ $\Leftrightarrow$ Plunging $(e, \ell)$

All conformal transformations relating the orbits in each class are detailed in Appendix B.3. The main usefulness of these conformal transformations lies in relating all complicated equatorial orbits to the simple NHEK or near-NHEK circular orbits. This remarkable property will enable us below to obtain analytically the gravitational wave emission of generic corotating equatorial orbits.

\section{Extremal perturbation theory}

Linear perturbations around the Kerr black hole are governed essentially by one separable partial differential equation, the Teukolsky master equation. A brief review of gravitational perturbations of Kerr as well as the conventions with respect to the NewmanPenrose formalism, in which this approach is formulated, can be found in Appendix A. The upshot of this is that we are interested in obtaining the Newman-Penrose scalar $\Psi_{(-2)}$ in the decomposition A.35.

\subsection{Gravitational perturbations of Kerr}

The near-extremality condition (2.1) guarantees the existence of a (near-)NHEK region of Kerr. This is equivalent to the condition that the reduced Hawking temperature

$$
\tau_{H}=\frac{\hat{r}_{+}-\hat{r}_{-}}{\hat{r}_{+}} \ll 1 .
$$

We are interested in gravitational wave emission from moving probes within the (near)NHEK region. Within this region, waves have an energy $\hat{\omega}>0$ and angular momentum $m$ close to the superradiant bound,

$$
M\left|\hat{\omega}-m \Omega_{H}\right| \ll 1
$$


Since we only consider such corotating modes, we restrict to corotating waves $m>0$ from now on. However, we allow for modes to be slightly below or slightly above the superradiant bound. In the terminology of the near-horizon region, we allow for positive or negative near-horizon energy.

The existence of these small parameters allows the use of the method of matched asymptotic expansions. In this, the near-horizon region is defined in terms of the adimensional Boyer-Lindquist radius $\hat{x}$ as

$$
\hat{x} \equiv \frac{\hat{r}-\hat{r}_{+}}{\hat{r}_{+}} \ll 1 .
$$

The radial coordinate which resolves the near-horizon region is taken to be either the NHEK radius $R$, defined as $\hat{x}=\lambda^{2 / 3} R$, or the near-NHEK radius $r$, defined as $\hat{x}=\frac{\lambda}{\kappa} r$ (where $\kappa>0$ is any fixed constant) as described in Section 2 .

The asymptotic or 'far' region is defined as

$$
\hat{x} \gg \frac{\hat{r}_{+}-\hat{r}_{-}}{\hat{r}_{+}}, \quad \hat{x} \gg M\left|\hat{\omega}-m \Omega_{H}\right|,
$$

and the intermediate or matching region is defined as

$$
\max \left(\frac{\hat{r}_{+}-\hat{r}_{-}}{\hat{r}_{+}}, M\left|\hat{\omega}-m \Omega_{H}\right|\right) \ll \hat{x} \ll 1 .
$$

We impose the boundary condition that the solution of the Teukolsky equation is outgoing at asymptotic null infinity and ingoing at the horizon. The stress-tensor source is taken to be a non-spinning point particle of rest mass $m_{0}$ following a geodesic $x_{*}^{\alpha}(\tau)$ inside the near-horizon region,

$$
T^{\mu \nu}=m_{0} \int \frac{d \tau}{\sqrt{-g}} \frac{d x^{\mu}}{d \tau} \frac{d x^{\nu}}{d \tau} \delta^{(4)}\left(x^{\alpha}-x_{*}^{\alpha}(\tau)\right) .
$$

We now describe the general solution, up to an undetermined coefficient $B$ defined below, which is dictated by the near-horizon physics. The latter requires an explicit computation that depends on the specific geodesic and is described in Section 5.

Asymptotic region: At zeroth order approximation in the near-extremal (4.1) and near-superadiant limit (4.2), the only relevant spheroidal harmonics A.36 are those with

$$
\hat{\omega}=m \Omega_{e x t}=\frac{m}{2 M}
$$

thereby leading to the extremal spheroidal harmonics defined in A.46- A.47). In the far region (4.4), the radial equation A.38 can be approximated by

$$
\hat{x}^{-2 s} \frac{\mathrm{d}}{\mathrm{d} \hat{x}}\left(\hat{x}^{2 s+2} \frac{\mathrm{d} \hat{R}_{l m \hat{\omega}}^{\mathrm{far}}}{\mathrm{d} \hat{x}}\right)+\left[\frac{m^{2}}{4}(\hat{x}+2)^{2}+i s m \hat{x}+\frac{3}{4} m^{2}+s(s+1)-\mathcal{E}_{l m}\right] \hat{R}_{l m \hat{\omega}}^{\mathrm{far}}=0
$$


where $\hat{R}_{l m \hat{\omega}}^{\text {far }}(\hat{x})=\hat{R}_{l m \hat{\omega}}\left(\hat{r}_{+}(1+\hat{x})\right)$, which agrees with [28]. The solutions can be expressed in terms of confluent hypergeometric functions

$$
\hat{R}_{l m \hat{\omega}}^{\mathrm{far}}(\hat{x})=P \hat{x}^{h-1-s} e^{-i m \hat{x} / 2}{ }_{1} F_{1}(h+i m-s, 2 h, i m \hat{x})+Q(h \mapsto 1-h)
$$

where $(h \mapsto 1-h)$ indicates that we should replace $h$ by $1-h$ in the first term and we defined the weight

$$
h=\frac{1}{2} \pm \frac{1}{2} \eta_{l m}, \quad \eta_{l m} \equiv \sqrt{1-7 m^{2}+4 \mathcal{E}_{l m}}
$$

The sign is fixed such that $h>\frac{1}{2}$ when $\eta_{l m}^{2}>0$ and $\operatorname{Im}(h)<0$ when $\eta_{l m}^{2}<0^{9}$. The axisymmetric modes $(m=0)$ have $\eta_{l 0}=1+2 l$ and real weight $h=1+l$. For $|s|=2$, all modes with $l=2,3,4$ have a real weight except the modes at the extremities of the range: $|m|=l$ where it is complex. For high $l$ the modes with approximately $|m|<0.75 l$ are real and those with $|m|>0.75 l$ are complex, see e.g. Figures 1 and 2 of [23].

The outgoing boundary condition at infinity implies

$$
P=-Q(-i m)^{2 h-1} \frac{\Gamma(2-2 h)}{\Gamma(2 h)} \frac{\Gamma(h-i m+s)}{\Gamma(1-h-i m+s)}
$$

such that

$$
\begin{aligned}
\hat{R}_{l m \hat{\omega}}^{\mathrm{far}}(\hat{x} \rightarrow \infty) & =Q \frac{\Gamma(2-2 h)}{\Gamma(1-h+i m-s)}(i m)^{h-1+i m-s} \\
& \times\left[1-\frac{(-i m)^{2 h-1}}{(i m)^{2 h-1}} \frac{\sin \pi(h+i m)}{\sin \pi(h-i m)}\right] \hat{x}^{-1+i m-2 s} e^{i m \hat{x} / 2}, \\
\hat{R}_{l m \hat{\omega}}^{\mathrm{far}}(\hat{x} \rightarrow 0) & =P \hat{x}^{h-1-s}\left(1+O\left(\hat{x}^{1}\right)\right)+Q \hat{x}^{-h-s}\left(1+O\left(\hat{x}^{1}\right)\right) .
\end{aligned}
$$

In terms of the radial tortoise coordinate $\hat{r}^{*}$ defined in 2.4 we have $2^{-i m} e^{i m / 2} \hat{x}^{i m} e^{i m \hat{x} / 2}=$ $e^{i \hat{\omega} \hat{r}_{*}}+O\left(\hat{x}^{-1}\right)$ after using (4.7) and $\Psi_{(s)} \sim \hat{x}^{-1-2 s} e^{i \hat{\omega}\left(-\hat{t}+\hat{r}_{*}\right)+i m \hat{\phi}}$ is indeed outgoing. The remaining constant $Q$ is determined by matching onto the near-horizon solution in the matching region.

Near-horizon region (NHEK): The Newman-Penrose scalar $\Psi_{(-2)}$ A.35 is expressed in terms of extremal spheroidal harmonics defined in A.47). In order to solve for the radial behavior, let us first rewrite the wave perturbation in terms of variables adapted to the NHEK region. Following $(2.7)$ we have $e^{-i \hat{\omega} \hat{t}+i m \hat{\phi}}=e^{-i \Omega T+i m \Phi}$ where

$$
\Omega \equiv \frac{2 M}{\lambda^{2 / 3}}\left(\hat{\omega}-\frac{m}{2 M}\right)
$$

\footnotetext{
${ }^{9}$ This matches with the convention of 37 (after identifying their $\delta$ as our $-i\left(h-\frac{1}{2}\right)$ ) but differs
} from 28$]$ if $\Im(h) \neq 0$. Our definition of $\eta_{l m}$ coincides with $\eta$ in 23]. 
is the near-horizon frequency. From the point of view of a NHEK observer, a finite energy perturbation in the asymptotically flat region has diverging energy in the extremal limit. Substituting $\hat{\omega}$ from (4.14) and using the change of coordinates (2.7), the radial equation A.38 exactly reduces in the limit $\lambda \rightarrow 0$ to the NHEK radial equation A.53) for $R_{l m \Omega}$, with

$$
R_{l m \Omega}(R)=\hat{R}_{l m \hat{\omega}}\left(M+M \lambda^{2 / 3} R\right), \quad T_{l m \Omega}(R)=\hat{T}_{l m \hat{\omega}}\left(M+M \lambda^{2 / 3} R\right) .
$$

The homogeneous solution to the NHEK radial equation is given in terms of Whittaker functions as a linear combination of

$$
\begin{aligned}
& \mathcal{M}_{l m \Omega}^{\mathrm{D}}(R)=R^{-s} M_{i m+s, h-1 / 2}\left(\frac{-2 i \Omega}{R}\right), \\
& \mathcal{W}_{l m \Omega}^{\text {in }}(R)=R^{-s} W_{i m+s, h-1 / 2}\left(\frac{-2 i \Omega}{R}\right),
\end{aligned}
$$

where the parameter $h$ was already defined in the asymptotic region in (4.10). These functions have a special behavior at infinity and at the horizon, respectively. The function $\mathcal{W}_{l m \Omega}^{\text {in }} \rightarrow(-2 i \Omega)^{i m+s} R^{-i m-2 s} e^{i \Omega / R}$ is purely ingoing at the horizon $R \rightarrow 0$ while for $R \rightarrow \infty$

$$
\begin{aligned}
& \mathcal{M}_{l m \Omega}^{\mathrm{D}}=(-2 i \Omega)^{h} R^{-h-s}\left(1+O\left(R^{-1}\right)\right), \\
& \mathcal{W}_{l m \Omega}^{\text {in }}=c_{h}^{\Omega, m} R^{-h-s}\left(1+O\left(R^{-1}\right)\right)+c_{1-h}^{\Omega, m} R^{h-1-s}\left(1+O\left(R^{-1}\right)\right),
\end{aligned}
$$

with $c_{h}^{\Omega, m}=(-2 i \Omega)^{h} \frac{\Gamma(1-2 h)}{\Gamma(1-h-i m-s)}$. The function $\mathcal{M}^{\mathrm{D}}$ therefore obeys Dirichlet boundary conditions.

Away from the source, for radii $R>R_{*}(\tau)$ where $R_{*}(\tau)$ describes the source geodesic, the radial function is a solution to the homogenous equation and can be written in terms of two coefficients $A$ and $B$ as

$$
\left.R_{l m \Omega}(R)\right|_{R>R_{*}(\tau)}=A \mathcal{W}_{l m \Omega}^{\mathrm{in}}(R)+B \mathcal{M}_{l m \Omega}^{\mathrm{D}}(R) .
$$

The "near-horizon coefficients" $A$ and $B$ can be matched onto the asymptotic coefficients $P, Q$ through the matching conditions

$$
\left.\delta \psi_{4}\right|_{K e r r}=M^{2} \lambda^{4 / 3} \times\left.\delta \psi_{4}\right|_{N H E K}
$$

where the prefactor originates from the type III rotation from the Kerr tetrad frame to the NHEK tetrad (A.16), which induces a scaling of the Newman-Penrose scalar A.16). After performing the substitution A.35 and A.51, the matching condition can be rewritten in terms of the radial functions as

$$
\lim _{\hat{x} \rightarrow 0} \hat{R}_{l m \hat{\omega}}^{f a r}(\hat{x})=M^{4} \times \frac{2 M}{\lambda^{2 / 3}} \times M^{2} \lambda^{4 / 3} \times\left.\lim _{R \rightarrow \infty} R_{l m \Omega}(R)\right|_{R>R_{*}(\tau)}
$$


where the extra $M^{4}$ factor comes from the difference between $\rho \sqrt{\mathrm{A} .20}$ ) and $\eta(\mathrm{A} .33$ and the $2 M \lambda^{-2 / 3}$ factor from the difference in frequency integration (4.14). Using (4.13) and $\hat{x}=\lambda^{2 / 3} R+o\left(\lambda^{2 / 3}\right)$ from (2.7) we find

$$
\begin{aligned}
& A=-\frac{Q \lambda^{\frac{2}{3}}}{2 M^{7}}(-i m)^{2 h-1}\left(-2 i \Omega \lambda^{2 / 3}\right)^{h-1} \frac{\Gamma(2-2 h)}{\Gamma(2 h-1) \Gamma(2 h)} \frac{\Gamma(h-i m+s) \Gamma(h-i m-s)}{\Gamma(1-h-i m+s)}, \\
& Q=2 M^{7} \lambda^{-\frac{2}{3}} B\left(-2 i \lambda^{2 / 3} \Omega\right)^{h} \times \\
& \left(1-\left(-i \lambda^{2 / 3} \Omega\right)^{2 h-1}(-2 i m)^{2 h-1} \frac{\Gamma(1-2 h)^{2}}{\Gamma(2 h-1)^{2}} \frac{\Gamma(h-i m+s)}{\Gamma(1-h-i m+s)} \frac{\Gamma(h-i m-s)}{\Gamma(1-h-i m-s)}\right)^{-1}
\end{aligned}
$$

The only remaining unknown is $B$, which will be fixed after we find the particular solution for the relevant source.

In summary, in the Kinnersley tetrad adapted to the Kerr geometry, the asymptotic behavior of the Newman-Penrose scalar $\delta \psi_{4}=\rho^{4} \Psi_{(-2)}$ (where $\rho$ is given in A.20) and $\Psi_{(-2)}$ in $\left.\mathrm{A} .35\right)$ reads

$$
\delta \psi_{4}(\hat{r} \rightarrow \infty)=\frac{M^{2}}{\sqrt{2 \pi}} \int_{-\infty}^{\infty} \mathrm{d} \Omega \sum_{l m} B \mathcal{K} S_{l m}(\theta) e^{i m \hat{\phi}} e^{-\frac{i}{2 M}\left(m+\lambda^{2 / 3} \Omega\right) \hat{u}} \hat{x}^{-1} .
$$

Here we have used (4.12)-(4.22), and we have defined the asymptotic retarded time $\hat{u}=$ $\hat{t}-\hat{r}^{*}$. In this expression

$$
\begin{aligned}
\mathcal{K} & \equiv \frac{\left(-2 i \lambda^{2 / 3} \Omega\right)^{h} k_{1}}{1-\left(-i \lambda^{2 / 3} \Omega\right)^{2 h-1} k_{2}}, \\
k_{1} & \equiv \frac{2^{i m} e^{-i m / 2} \Gamma(2-2 h)}{\Gamma(1-h+i m-s)}(i m)^{h-1+i m-s}\left[1-\frac{(-i m)^{2 h-1}}{(i m)^{2 h-1}} \frac{\sin \pi(h+i m)}{\sin \pi(h-i m)}\right], \\
k_{2} & \equiv(-2 i m)^{2 h-1} \frac{\Gamma(1-2 h)^{2}}{\Gamma(2 h-1)^{2}} \frac{\Gamma(h-i m+s)}{\Gamma(1-h-i m+s)} \frac{\Gamma(h-i m-s)}{\Gamma(1-h-i m-s)},
\end{aligned}
$$

and $s=-2$ is understood.

Note that the existence of a matching region 4.5 requires $\Omega \lambda^{2 / 3} \ll 1$. Therefore care must be taken when evaluating the Fourier integral (4.23) in the small $\lambda$ limit. We return to this point below. Note also that all modes with $\operatorname{Re}(h) \gg 1$ are highly suppressed. The leading contributions to 4.23 will come from the complex modes $h=\frac{1}{2}+i h_{I}$.

Near-horizon region (near-NHEK): Alternatively, if the source is located within the near-NHEK region, we need to match the asymptotically flat solution to the near-NHEK solution. Due to 2.9 , we have $e^{-i \hat{\omega} \hat{t}+i m \hat{\phi}}=e^{-i \omega t+i m \phi}$ with

$$
\omega=\frac{2 M \kappa}{\lambda}\left(\hat{\omega}-\frac{m}{2 M}\right) .
$$


Using this expression for $\hat{\omega}$ and the change of coordinates 2.9 , the radial equation A.38 reduces to the near-NHEK radial equation A.48 in the limit $\lambda \rightarrow 0$ upon identifying

$$
R_{l m \omega}(r)=\hat{R}_{l m \hat{\omega}}\left(M+M \frac{\lambda}{\kappa}(r+\kappa)\right), \quad T_{l m \omega}(r)=\hat{T}_{l m \hat{\omega}}\left(M+M \frac{\lambda}{\kappa}(r+\kappa)\right) .
$$

The homogeneous solutions to the radial near-NHEK equation A.48 are spanned by

$$
\begin{gathered}
\mathcal{R}^{\mathrm{in}}(r)=r^{-i n / 2-s}\left(\frac{r}{2 \kappa}+1\right)^{i\left(\frac{n}{2}-m\right)-s}{ }_{2} F_{1}\left(h-i m-s, 1-h-i m-s, 1-i n-s,-\frac{r}{2 \kappa}\right), \\
\mathcal{R}^{\mathrm{D}}(r)=r^{-h-s}\left(\frac{2 \kappa}{r}+1\right)^{i\left(\frac{n}{2}-m\right)-s}{ }_{2} F_{1}\left(h-i m-s, h-i m+i n, 2 h,-\frac{2 \kappa}{r}\right)
\end{gathered}
$$

where we defined

$$
n=\frac{\omega}{\kappa}+m
$$

The boundary conditions satisfied by these solutions are again respectively ingoing and Dirichlet. More precisely,

$$
\begin{aligned}
& \mathcal{R}^{\mathrm{D}}(r)=r^{-h-s}\left(1+O\left(r^{-1}\right)\right), \\
& \mathcal{R}^{\text {in }}(r)=d_{h}^{m, n} r^{-h-s}\left(1+O\left(r^{-1}\right)\right)+d_{1-h}^{n, m} r^{h-1-s}\left(1+O\left(r^{-1}\right)\right)
\end{aligned}
$$

with $d_{h}^{n, m}=(2 \kappa)^{h-\frac{i n}{2}} \frac{\Gamma(1-2 h) \Gamma(1-i n-s)}{\Gamma(1-h+i(m-n)) \Gamma(1-h-i m-s)}$.

Outside the source, which is described by $r_{*}(\tau)$, the radial function is again a solution to the homogeneous equation and it can be written as

$$
\left.R_{l m \omega}(r)\right|_{r>r_{*}(\tau)}=A \mathcal{R}^{\mathrm{in}}(r)+B \mathcal{R}^{\mathrm{D}}(r) .
$$

Matching this solution to the far solution using (4.13) and $\hat{x}=\frac{\lambda}{\kappa} r$ from (2.9) we find

$$
\begin{aligned}
A & =-\frac{Q}{2 M^{7}} 2^{h+i n / 2-1}(-i m)^{2 h-1} \lambda^{h} \kappa^{i n / 2-1} \\
& \times \frac{\Gamma(2-2 h)}{\Gamma(2 h-1) \Gamma(2 h)} \frac{\Gamma(h-i m+s) \Gamma(h-i m-s) \Gamma(h-i(n-m))}{\Gamma(1-h-i m+s) \Gamma(1-i n-s)} \\
Q & =2 M^{7} \kappa^{1-h} \lambda^{-1+h} B \\
& \times\left(1-(-2 i m \lambda)^{2 h-1} \frac{\Gamma(1-2 h)^{2}}{\Gamma(2 h-1)^{2}} \frac{\Gamma(h-i m+s)}{\Gamma(1-h-i m+s)} \frac{\Gamma(h-i m-s) \Gamma(h-i n+i m)}{\Gamma(1-h-i m-s) \Gamma(1-h-i n+i m)}\right)^{-1}
\end{aligned}
$$

where $s=-2$ is understood. Therefore,

$$
\delta \psi_{4}(\hat{r} \rightarrow \infty)=\frac{M^{2}}{\sqrt{2 \pi}} \int_{-\infty}^{\infty} \mathrm{d} \omega \sum_{l m} B \mathcal{K}_{\kappa} S_{l m}(\theta) e^{i m \hat{\phi}} e^{-i \hat{\omega} \hat{u}} \hat{x}^{-1}
$$

with

$$
\mathcal{K}_{\kappa} \equiv \frac{\lambda^{h} \kappa^{-h} k_{1}}{1-\lambda^{2 h-1} k_{2} \frac{\Gamma(h-i n+i m)}{\Gamma(1-h-i n+i m)}} .
$$




\subsection{Quasi-normal mode approximation}

So far, we have given frequency-based waveforms $(4.23)$ and 4.35 for the curvature perturbation. Experiment however requires time-based waveforms. In what follows we ignore the short time transients, which are primarily associated with the motion of the source, and the tail at very late times due to the further scattering of gravitational waves. Instead we focus on the contributions from the quasi-normal modes (QNM), which provide a good approximation to the waveform at late (but not very late) times. To select the QNM contribution we deform the frequency integrals over the real axis in 4.23 and 4.35 in the lower complex plane and rewrite this as a sum of three components: the branch cut corresponding to the tail at very late times [42], the half lower circle corresponding to the short time transients and finally the sum over quasi-normal modes that accounts for the late-time behavior of the waveform.

The spectrum of Kerr quasi-normal modes bifurcates in the near-extremal limit into "zero-damped" and "damped" quasi-normal modes [37]. The damped QNMs decouple from the near-horizon region and can therefore be ignored. The zero-damped QNMs was observed by Hod [43] to match the analytic formulae

$$
\begin{aligned}
\operatorname{Re}\left(\hat{\omega}_{N l m}\right) & =\frac{m}{2 M}+o\left(\lambda^{0}\right), \\
\operatorname{Im}\left(\hat{\omega}_{N l m}\right) & =-i \frac{2 \pi T_{H}}{2 M \Omega_{H}}\left(N+\frac{1}{2}\right)+o(\lambda)=-i \frac{\lambda}{2 M}\left(N+\frac{1}{2}\right)+o(\lambda)
\end{aligned}
$$

where $N=0,1,2, \ldots$ is the overtone number, up to small corrections and can be written more precisely at first order in the near-extremal limit as 37 (see also [44])

$$
\hat{\omega}_{N l m}=\frac{1}{2 M}(m-i \lambda(N+h))+o(\lambda)
$$

where $h$ is given by (4.10). The QNMs with $\eta_{l m}^{2}>0$ are usually called the normal modes, and the QNMs with $\eta_{l m}^{2}<0$ are the travelling waves. The real part of $\hat{\omega}$ is lower than $\frac{m}{2 M}$ for all travelling waves.

Given the scaling in $\lambda$ of 4.38 and the absence of other QNMs with an intermediate $\lambda^{p}, 0<p<1$ scaling (see however [45, 46]), we conclude there is no QNM in the NHEK limit. However there are the damped QNMs in the near-NHEK limit. We are therefore allowed to use the QNM approximation when the source is in the near-NHEK region, where the formula (4.35) applies. Using (4.27) the near-NHEK quasi-normal frequencies are

$$
\omega_{N l m}=-i \kappa(N+h) .
$$

\footnotetext{
${ }^{10}$ Our convention for $h \sqrt{4.10}$ is crucial for the validity of this formula. This expression was also obtained in 29] for modes with $\eta^{2}>0$. Note that in 37], it was discussed that this formula is not a good approximation for small but finite $\lambda$ for specific modes $l, m$.
} 
The QNM (4.39) originate from poles of $\Gamma(h-i n+i m)$. It is important to note that the coefficient $B$ is proportional to $\Gamma(h-i n+i m)$. Indeed, $B$ is given by the Green function constructed from the homogeneous solutions as

$$
B=\frac{1}{\tilde{W}} \int_{0}^{\infty} \mathrm{d} r^{\prime} \mathcal{R}^{\mathrm{in}}\left(r^{\prime}\right) \frac{T_{l m \omega}\left(r^{\prime}\right)}{r^{\prime}\left(r^{\prime}+2 \kappa\right)}
$$

where the Wronskian is

$$
\tilde{W}=-\frac{\Gamma(2 h) \Gamma(1-i n-s)}{\Gamma(h-i m-s) \Gamma(h-i n+i m)}(2 \kappa)^{1-h-i n / 2} .
$$

The residue of the QNM can be obtained by expanding $B \mathcal{K}_{\kappa}$ (4.36) around $\omega=\omega_{N l m}$,

$$
B \mathcal{K}_{\kappa}=k_{1}\left(\frac{\lambda}{\kappa}\right)^{h} \frac{B}{\Gamma(h-i n+i m)} \frac{i \kappa}{\omega-\omega_{N l m}} \frac{(-1)^{N}}{N !} .
$$

For normal modes this is immediate. For travelling waves one has to note that $k_{2} \ll 1$ (with some exceptions such as the $(l, m)=(9,7)$ or $(13,10)$ modes where $\left|k_{2}\right| \approx 10^{-4}$, see also [37]). In those cases, the formula 44.42 is therefore an approximation.

Considering the contribution of these modes only, the residue theorem yields

$$
\begin{aligned}
\delta \psi_{4}(\hat{r} \rightarrow \infty)= & \sqrt{2 \pi} M^{2} \sum_{l m} \lambda^{h} \kappa^{1-h} k_{1} S_{l m}(\theta) e^{i m \hat{\phi}} \hat{x}^{-1} \\
& \times\left.\sum_{N} \frac{(-1)^{N} e^{-i \hat{\omega}_{N l m} \hat{u}}}{N !} \frac{B}{\Gamma(h-i n+i m)}\right|_{\omega=\omega_{N l m}} .
\end{aligned}
$$

When the source $T_{l m \omega}(r)$ can be considered as independent of the overtone number $N$, i.e. $T_{l m \omega}(r)=T_{l m}(r)$, we can perform the overtone sum exactly. Gathering, in this approximation, the $N$-dependent part of 4.43 but neglecting the potential overtone dependence in the source results in the following overtone sum

$$
\sum_{N=0}^{\infty} \frac{(-1)^{N} e^{-\frac{\lambda N \hat{u}}{2 M}}\left(1+\frac{2 \kappa}{r^{\prime}}\right)^{\frac{N}{2}}}{N !} \frac{{ }_{2} F_{1}\left(h-i m-s, 1-h-i m-s, 1-h-i m-s-N ;-\frac{r^{\prime}}{2 \kappa}\right)}{\Gamma(1-h-i m-s-N)}
$$

which is computed in Appendix in D.19) with

$$
\begin{aligned}
x & =e^{-\frac{\lambda \hat{u}}{2 M}}\left(1+\frac{2 \kappa}{r^{\prime}}\right)^{\frac{1}{2}}, \quad z=\frac{r^{\prime}}{r^{\prime}+2 \kappa}, \\
c_{+} & =h+i m+s, \quad c_{-}=h-i m-s .
\end{aligned}
$$

It leads to

$$
\delta \psi_{4}(\hat{r} \rightarrow \infty)=\sqrt{2 \pi} M^{2} \sum_{l m} k_{1} \frac{\lambda^{h}}{\kappa^{h}} S_{l m}(\theta) e^{i m \hat{\phi}} e^{-i \frac{m-i \lambda h}{2 M} \hat{u}} \hat{x}^{-1} \int_{0}^{\infty} \mathrm{d} r^{\prime} \frac{T_{l m}\left(r^{\prime}\right)}{r^{\prime}\left(r^{\prime}+2 \kappa\right)} \mathcal{G}\left(\hat{u}, r^{\prime}\right)
$$


where the Green function, which connects the near-NHEK physics with the asymptotic observer, is given by

$$
\begin{aligned}
\mathcal{G}\left(\hat{u}, r^{\prime}\right) & \equiv-\frac{1}{2}(2 \kappa)^{\frac{3 h}{2}+\frac{i m}{2}} \frac{\Gamma(h-i m-s)}{\Gamma(2 h) \Gamma(1-h-i m-s)} r^{-\frac{h}{2}-\frac{i m}{2}-s}\left(1+\frac{r^{\prime}}{2 \kappa}\right)^{-\frac{h}{2}+\frac{i m}{2}} \\
& \times\left(1-\left(1+\frac{2 \kappa}{r^{\prime}}\right)^{-\frac{1}{2}} e^{-\frac{\lambda \hat{u}}{2 M}}\right)^{-h+i m+s}\left(1-\left(1+\frac{2 \kappa}{r^{\prime}}\right)^{+\frac{1}{2}} e^{-\frac{\lambda \hat{u}}{2 M}}\right)^{-h-i m-s}
\end{aligned}
$$

This general expression for the emission illustrates the possible phenomenology of general trajectories in near-NHEK spacetime. For retarded times in the range $0<\frac{\lambda \hat{u}}{2 M}<1$ of the asymptotic observer, we can approximate $1-e^{-\frac{\lambda \hat{u}}{2 M}} \approx \frac{\lambda \hat{u}}{2 M}$ and we observe that $\delta \psi_{4}$ exhibits a fall-off in between $\hat{u}^{-h}$ and $\hat{u}^{-2 h}$, depending on the details of the integral of the Green function. The dominant modes are the travelling waves with $\operatorname{Re}(h)=\frac{1}{2}$. The behavior $\hat{u}^{-1}$ for such modes in the limit of a source in the region $r^{\prime} \rightarrow \infty$ has been predicted before [37] and it was observed numerically [47]. By contrast, the intermediate behavior $\hat{u}^{-\chi}$, with $1 / 2 \leq \chi \leq 1$, remains to be found numerically although the full expression for the (near-horizon) QNM approximation to the Green function has also been derived before 35] 11. In Section 6.4 we discuss explicit trajectories where this behavior is found. In light of (4.47), this implies a significant contribution to the gravitational wave signal from deep inside the near-NHEK region.

\section{Emission from circular near-NHEK orbits}

The spectrum of emission of a body moving on a circular geodesic in NHEK spacetime at first order in the asymptotically matched expansion was obtained in [13,28] by the Green's function method. We repeat this computation in our notation in Appendix $\mathrm{C}$ and find complete agreement, up to a global sign. This global sign difference originates from the sign of the stress-tensor in Teukolsky equations A.31. This global sign difference leads to phase shift which does not modify the amplitude or energy fluxes of [28], which have been confirmed numerically independently 13 .

In this section, we extend the analysis to the case of (unstable) circular orbits in nearNHEK spacetime. The procedure applied to circular orbits in near-NHEK is essentially the same as in NHEK and we invite the interested reader to first review the computation in Appendix C. In what follows we need both $\delta \psi_{0}$ and $\delta \psi_{4}$ so we calculate both quantities.

Separating the perturbation equation as in A.44 and specializing to circular orbits

\footnotetext{
${ }^{11}$ Our result matches theirs taking into account that a different tetrad was used and that the overtone dependence of the source was neglected.
} 
(B.14) we have

$$
\begin{aligned}
\delta \psi_{4} & =\frac{1}{(1-i \cos \theta)^{4}} \sum_{l m} R_{l m \tilde{\omega}}^{(s=-2)}(r) S_{l m}^{(s=-2)}(\theta) e^{i m(\phi-\tilde{\omega} t)} \\
\delta \psi_{0} & =\sum_{l m} R_{l m \tilde{\omega}}^{(s=2)}(r) S_{l m}^{(s=2)}(\theta) e^{i m(\phi-\tilde{\omega} t)}
\end{aligned}
$$

with

$$
\tilde{\omega}=-\frac{3 r_{0}}{4}\left(1+\kappa_{0}\right), \quad \kappa_{0} \equiv \frac{\kappa}{r_{0}}=\left(\frac{2 \ell}{\sqrt{3\left(\ell^{2}-\ell_{*}^{2}\right)}}-1\right)^{-1}
$$

where we used (B.14). Note the bound $0<\kappa_{0}<\frac{\sqrt{3}}{2-\sqrt{3}}$. It reflects that the timelike circular orbits in the near-NHEK region lie in between the ISCO $r_{0} \rightarrow \infty$ (or $\ell \rightarrow \ell_{*}$ ) and the photon circular orbit $r_{0} \rightarrow \kappa\left(\frac{2}{\sqrt{3}}-1\right)$ (or $\left.\ell \rightarrow \infty\right)$. The spheroidal harmonics are independent of the sign of the spin $s$ so we drop their superscript from now on.

The source term for $s=-2$ is given by

$$
\begin{aligned}
\mathcal{T}_{4} & =\frac{m_{0} r_{0}^{3}}{64 M^{7} \sqrt{3\left(1+2 \kappa_{0}\right)-\kappa_{0}^{2}}} \\
\times & \left\{144\left[1+4 \kappa_{0}+\frac{61}{18} \kappa_{0}^{2}-\frac{11}{9} \kappa_{0}^{3}+\frac{1}{9} \kappa_{0}^{4}\right] \delta\left(r-r_{0}\right) \delta\left(\theta-\frac{\pi}{2}\right) \delta(\phi-\tilde{\omega} t)\right. \\
+ & 16\left[1+5 \kappa_{0}+7 \kappa_{0}^{2}+\kappa_{0}^{3}-2 \kappa_{0}^{4}\right] r_{0} \delta^{\prime}\left(r-r_{0}\right) \delta\left(\theta-\frac{\pi}{2}\right) \delta(\phi-\tilde{\omega} t) \\
- & 48 i\left[1+4 \kappa_{0}+\frac{10}{3} \kappa_{0}^{2}-\frac{4}{3} \kappa_{0}^{3}\right] \delta\left(r-r_{0}\right) \delta^{\prime}\left(\theta-\frac{\pi}{2}\right) \delta(\phi-\tilde{\omega} t) \\
- & 21\left[1+4 \kappa_{0}+\frac{68}{21} \kappa_{0}^{2}-\frac{32}{21} \kappa_{0}^{3}+\frac{1}{7} \kappa_{0}^{4}\right] \delta\left(r-r_{0}\right) \delta\left(\theta-\frac{\pi}{2}\right) \delta^{\prime}(\phi-\tilde{\omega} t) \\
& -8 i\left(1+\kappa_{0}\right)\left(1+2 \kappa_{0}\right)^{2} r_{0} \delta^{\prime}\left(r-r_{0}\right) \delta^{\prime}\left(\theta-\frac{\pi}{2}\right) \delta(\phi-\tilde{\omega} t) \\
- & 3\left[1+5 \kappa_{0}+\frac{23}{3} \kappa_{0}^{2}+3 \kappa_{0}^{3}-\frac{2}{3} \kappa_{0}^{4}\right] r_{0} \delta^{\prime}\left(r-r_{0}\right) \delta\left(\theta-\frac{\pi}{2}\right) \delta^{\prime}(\phi-\tilde{\omega} t) \\
+ & 6 i\left[1+4 \kappa_{0}+\frac{11}{3} \kappa_{0}^{2}-\frac{2}{3} \kappa_{0}^{3}\right] \delta\left(r-r_{0}\right) \delta^{\prime}\left(\theta-\frac{\pi}{2}\right) \delta^{\prime}(\phi-\tilde{\omega} t) \\
+ & 2\left(1+\kappa_{0}\right)^{2}\left(1+2 \kappa_{0}\right)^{2} r_{0}^{2} \delta^{\prime \prime}\left(r-r_{0}\right) \delta\left(\theta-\frac{\pi}{2}\right) \delta(\phi-\tilde{\omega} t) \\
& -8\left(1+2 \kappa_{0}\right)^{2} \delta\left(r-r_{0}\right) \delta^{\prime \prime}\left(\theta-\frac{\pi}{2}\right) \delta(\phi-\tilde{\omega} t) \\
+ & \left.\frac{9}{8}\left(1+2 \kappa_{0}-\frac{1}{3} \kappa_{0}^{2}\right)^{2} \delta\left(r-r_{0}\right) \delta\left(\theta-\frac{\pi}{2}\right) \delta^{\prime \prime}(\phi-\tilde{\omega} t)\right\}
\end{aligned}
$$


and for $s=2$ it is given by

$$
\begin{aligned}
& \mathcal{T}_{0}=\frac{m_{0}}{128 M^{3} r_{0} \sqrt{3\left(1+2 \kappa_{0}\right)-\kappa_{0}^{2}}}\left[192 \delta\left(r-r_{0}\right) \delta\left(\theta-\frac{\pi}{2}\right) \delta(\phi-\tilde{\omega} t)\right. \\
& +\frac{8\left(9+\kappa_{0}\left(36+36 \kappa_{0}-\kappa_{0}^{3}\right)\right.}{\left(1+2 \kappa_{0}\right)^{2}} \delta\left(r-r_{0}\right) \delta\left(\theta-\frac{\pi}{2}\right) \delta^{\prime}(\phi-\tilde{\omega} t)+ \\
& \frac{\left(-3+\left(-6+\kappa_{0}\right) \kappa_{0}\right)^{2}}{\left(1+2 \kappa_{0}\right)^{2}} \delta\left(r-r_{0}\right) \delta\left(\theta-\frac{\pi}{2}\right) \delta^{\prime \prime}(\phi-\tilde{\omega} t)-128 i \delta\left(r-r_{0}\right) \delta^{\prime}\left(\theta-\frac{\pi}{2}\right) \delta(\phi-\tilde{\omega} t)+ \\
& \frac{16 i\left(-3+\left(-6+\kappa_{0}\right) \kappa_{0}\right)}{1+2 \kappa_{0}} \delta\left(r-r_{0}\right) \delta^{\prime}\left(\theta-\frac{\pi}{2}\right) \delta^{\prime}(\phi-\tilde{\omega} t)-64 \delta\left(r-r_{0}\right) \delta^{\prime \prime}\left(\theta-\frac{\pi}{2}\right) \delta(\phi-\tilde{\omega} t) \\
& -\frac{8 r_{0}\left(1+\kappa_{0}\right)\left(-3+\left(-6+\kappa_{0}\right) \kappa_{0}\right)}{1+2 \kappa_{0}} \delta^{\prime}\left(r-r_{0}\right) \delta\left(\theta-\frac{\pi}{2}\right) \delta^{\prime}(\phi-\tilde{\omega} t) \\
& \left.-64 i r_{0}\left(1+\kappa_{0}\right) \delta^{\prime}\left(r-r_{0}\right) \delta^{\prime}\left(\theta-\frac{\pi}{2}\right) \delta(\phi-\tilde{\omega} t)+16 r_{0}^{2}\left(1+\kappa_{0}\right)^{2} \delta^{\prime \prime}\left(r-r_{0}\right) \delta\left(\theta-\frac{\pi}{2}\right) \delta(\phi-\tilde{\omega} t)\right] .
\end{aligned}
$$

It can be remarked that $\mathcal{T}_{4}$ and $\mathcal{T}_{0}$ are related as follows

$$
\mathcal{T}_{4}=\left.\frac{r^{2}(r+2 \kappa)^{2}}{4 M^{4}\left(1+\cos ^{2} \theta\right)}\left(\frac{1+i \cos \theta}{1-i \cos \theta}\right)^{2} \mathcal{T}_{0}\right|_{(t, \phi) \rightarrow(-t,-\phi)}
$$

due to the invariance of $T_{\mu \nu}$ under $(t, \phi) \rightarrow(-t,-\phi)$. A formula analogous to (C.8) applies to near-NHEK upon adapting the notation such that

$$
T_{l m \tilde{\omega}}^{(s=-2)}(r)=\tilde{a}_{0} \delta\left(r-r_{0}\right)+\tilde{a}_{1} r_{0} \delta^{\prime}\left(r-r_{0}\right)+\tilde{a}_{2} r_{0}^{2} \delta^{\prime \prime}\left(r-r_{0}\right)
$$

with

$$
\begin{aligned}
\tilde{a_{0}} & =-\frac{m_{0} r_{0}^{3}}{16 M^{5} \sqrt{-\kappa_{0}^{2}+3\left(1+2 \kappa_{0}\right)}}\left(S _ { l m } ( \frac { \pi } { 2 } ) \left(-\frac{m^{2}}{8}\left(-3-6 \kappa_{0}+\kappa_{0}^{2}\right)^{2}\right.\right. \\
& \left.+8\left(4+16 \kappa_{0}+21 \kappa_{0}^{2}+10 \kappa_{0}^{3}+2 \kappa_{0}^{4}\right)+8\left(1+2 \kappa_{0}\right)^{2}-i m\left(-3-12 \kappa_{0}-20 \kappa_{0}^{2}-16 \kappa_{0}^{3}+3 \kappa_{0}^{4}\right)\right) \\
& \left.-2\left(1+2 \kappa_{0}\right)\left(m\left(-3-6 \kappa_{0}+\kappa_{0}^{2}\right)+8 i\left(1+2 \kappa_{0}+2 \kappa_{0}^{2}\right)\right) S_{l m}^{\prime}\left(\frac{\pi}{2}\right)-8\left(1+2 \kappa_{0}\right)^{2} S_{l m}^{\prime \prime}\left(\frac{\pi}{2}\right)\right), \\
\tilde{a_{1}} & =-\frac{m_{0} r_{0}^{3}}{16 M^{5} \sqrt{-\kappa_{0}^{2}+3\left(1+2 \kappa_{0}\right)}}\left(1+2 \kappa_{0}\right)\left(1+\kappa_{0}\right) \\
& \times\left(\left(-16-3 m i+2 \kappa_{0}(-16-3 m i)+\kappa_{0}^{2}(-16+i m)\right) S_{l m}\left(\frac{\pi}{2}\right)+8 i\left(1+2 \kappa_{0}\right) S_{l m}^{\prime}\left(\frac{\pi}{2}\right)\right), \\
\tilde{a_{2}} & =-\frac{m_{0} r_{0}^{3}}{8 M^{5}} \frac{\left(1+\kappa_{0}\right)^{2}\left(1+2 \kappa_{0}\right)^{2}}{\sqrt{-\kappa_{0}^{2}+3\left(1+2 \kappa_{0}\right)}} S_{l m}\left(\frac{\pi}{2}\right) .
\end{aligned}
$$

For the $s=2$ case, we can define

$$
T_{l m \tilde{\omega}}^{(s=2)}(r)=-4 M^{2} \int_{0}^{2 \pi} d \phi e^{-i m \phi} \int_{0}^{\pi} \sin \theta d \theta S_{l m}(\theta)\left(1+\cos ^{2} \theta\right) \mathcal{T}_{0} .
$$


Then we find

$$
T_{l m \tilde{\omega}}^{(s=2)}(r)=\tilde{\alpha}_{0} \delta\left(r-r_{0}\right)+\tilde{\alpha}_{1} r_{0} \delta^{\prime}\left(r-r_{0}\right)+\tilde{\alpha}_{2} r_{0}^{2} \delta^{\prime \prime}\left(r-r_{0}\right)
$$

with

$$
\begin{aligned}
\tilde{\alpha}_{0}= & \left(\frac{m_{0}}{32 M r_{0}\left(1+2 \kappa_{0}\right)^{2} \sqrt{-\kappa_{0}^{2}+3\left(1+2 \kappa_{0}\right)}}\right)\left[\left(-128\left(1+2 \kappa_{0}\right)^{2}+m^{2}\left(-3+\left(-6+\kappa_{0}\right) \kappa_{0}\right)^{2}\right.\right. \\
& \left.+8 i m\left(-9+\kappa_{0}\left(-36-36 \kappa_{0}+\kappa_{0}^{3}\right)\right)\right) S_{l m}\left(\frac{\pi}{2}\right)+16\left(1+2 \kappa_{0}\right) \\
& \left.\times\left(-\left(8 i\left(1+2 \kappa_{0}\right)+m\left(-3+\left(-6+\kappa_{0}\right) \kappa_{0}\right)\right) S_{l m}^{\prime}\left(\frac{\pi}{2}\right)+4\left(1+2 \kappa_{0}\right) S_{l m}^{\prime \prime}\left(\frac{\pi}{2}\right)\right)\right], \\
\tilde{\alpha}_{1}= & \frac{i m_{0}\left(1+\kappa_{0}\right)\left[m\left(-3+\left(-6+\kappa_{0}\right) \kappa_{0}\right) S_{l m}\left(\frac{\pi}{2}\right)-8\left(1+2 \kappa_{0}\right) S_{l m}^{\prime}\left(\frac{\pi}{2}\right)\right]}{4 M r_{0} \sqrt{-\kappa_{0}^{2}+3\left(1+2 \kappa_{0}\right)}\left(1+2 \kappa_{0}\right)} \\
\tilde{\alpha}_{2}= & -\frac{m_{0}}{2 M r_{0} \sqrt{-\kappa_{0}^{2}+3\left(1+2 \kappa_{0}\right)}}\left(1+\kappa_{0}\right)^{2} S_{l m}\left(\frac{\pi}{2}\right) .
\end{aligned}
$$

Finally, the solution obeying Dirichlet boundary conditions and ingoing boundary conditions at the horizon reads

$$
R_{l m \tilde{\omega}}(r)=\frac{\left(r_{0}\left(r_{0}+2 \kappa\right)\right)^{s}}{\tilde{W}}\left(\tilde{\mathcal{X}} \Theta\left(r_{0}-r\right) \mathcal{R}^{\mathrm{in}}(r)+\tilde{\mathcal{Z}} \Theta\left(r-r_{0}\right) \mathcal{R}^{\mathrm{D}}(r)\right)+\frac{\tilde{a}_{2}}{1+2 \kappa_{0}} \delta\left(r-r_{0}\right)
$$

with

$$
\begin{aligned}
\tilde{W} & =\frac{(1-2 h) \Gamma(2 h-1) \Gamma(1-i n-s)}{\Gamma(h-i m-s) \Gamma(h-i(n-m))}(2 \kappa)^{1-h-i n / 2}, \\
\tilde{\mathcal{X}} & =r_{0} \mathcal{R}^{\mathrm{D} \prime}\left(r_{0}\right)\left(-\tilde{a}_{1}-2(-s+1) \tilde{a}_{2} \frac{1+\kappa_{0}}{1+2 \kappa_{0}}\right) \\
& +\mathcal{R}^{\mathrm{D}}\left(r_{0}\right)\left(\tilde{a}_{0}-2 s \tilde{a}_{1} \frac{1+\kappa_{0}}{1+2 \kappa_{0}}+2 s \frac{\tilde{a}_{2}}{1+2 \kappa_{0}}+4 s(s-1) \frac{\left(1+\kappa_{0}\right)^{2}}{\left(1+2 \kappa_{0}\right)^{2}} \tilde{a}_{2}+\frac{\tilde{a}_{2}}{1+2 \kappa_{0}} V\left(r_{0}\right)\right), \\
\tilde{\mathcal{Z}} & =r_{0} \mathcal{R}^{\text {in'}}\left(r_{0}\right)\left(-\tilde{a}_{1}-2(-s+1) \tilde{a}_{2} \frac{1+\kappa_{0}}{1+2 \kappa_{0}}\right) \\
& +\mathcal{R}^{\text {in }}\left(r_{0}\right)\left(\tilde{a}_{0}-2 s \tilde{a}_{1} \frac{1+\kappa_{0}}{1+2 \kappa_{0}}+2 s \frac{\tilde{a}_{2}}{1+2 \kappa_{0}}+4 s(s-1) \frac{\left(1+\kappa_{0}\right)^{2}}{\left(1+2 \kappa_{0}\right)^{2}} \tilde{a}_{2}+\frac{\tilde{a}_{2}}{1+2 \kappa_{0}} V\left(r_{0}\right)\right)
\end{aligned}
$$

where $V(r)$ is given in A.49 and

$$
n=m+\frac{m \tilde{\omega}}{\kappa}=\frac{m}{4}\left(1-\frac{3}{\kappa_{0}}\right) .
$$

As it turns out, we will also need the $s=2$ solution obeying Dirichlet boundary conditions and outgoing boundary conditions at the horizon (cf. Section 6.1). This solution reads

$$
R_{l m \tilde{\omega}}^{(s=2)}(r)=\frac{\left(r_{0}\left(r_{0}+2 \kappa\right)\right)^{s}}{\tilde{W}^{\prime}}\left(\tilde{\mathcal{X}}^{\prime} \Theta\left(r_{0}-r\right) \mathcal{R}^{\text {out }}(r)+\tilde{\mathcal{Z}}^{\prime} \Theta\left(r-r_{0}\right) \mathcal{R}^{\mathrm{D}}(r)\right)+\frac{\tilde{\alpha}_{2}}{1+2 \kappa_{0}} \delta\left(r-r_{0}\right)
$$


where the outgoing solution basis is chosen to be

$$
\mathcal{R}^{\text {out }}(r)=r^{\frac{i n}{2}}\left(1+\frac{r}{2 \kappa}\right)^{i\left(m-\frac{n}{2}\right)}{ }_{2} F_{1}\left(h+i m+s, 1-h+i m+s, 1+i n+s,-\frac{r}{2 \kappa}\right)
$$

where $s=2$ is understood and finally,

$$
\begin{aligned}
\tilde{W}^{\prime} & =\frac{\Gamma(2 h) \Gamma(1+i n+s)}{\Gamma(h-i m+i n) \Gamma(h+i m+s)}(2 \kappa)^{1-h+\frac{i n}{2}+s}, \\
\tilde{\mathcal{X}}^{\prime} & =r_{0} \mathcal{R}^{\mathrm{D} \prime}\left(r_{0}\right)\left(\tilde{\alpha}_{1}-2 \tilde{\alpha}_{2}(-1+s) \frac{1+\kappa_{0}}{1+2 \kappa_{0}}\right)+\mathcal{R}^{\mathrm{D}}\left(r_{0}\right) \\
& \times\left(-\tilde{\alpha}_{0}+2 s \tilde{\alpha}_{1} \frac{1+\kappa_{0}}{1+2 \kappa_{0}}+\tilde{\alpha}_{2}\left(-2 s \frac{1}{1+2 \kappa_{0}}+4 s(1-s) \frac{\left(1+\kappa_{0}\right)^{2}}{\left(1+2 \kappa_{0}\right)^{2}}\right)-\frac{\tilde{\alpha}_{2}}{1+2 \kappa_{0}} V\left(r_{0}\right)\right), \\
\tilde{\mathcal{Z}}^{\prime} & =r_{0} \mathcal{R}^{\text {out }}\left(r_{0}\right)\left(\tilde{\alpha}_{1}-2 \tilde{\alpha}_{2}(-1+s) \frac{1+\kappa_{0}}{1+2 \kappa_{0}}\right)+\mathcal{R}^{\text {out }}\left(r_{0}\right) \\
& \times\left(-\tilde{\alpha}_{0}+2 s \tilde{\alpha}_{1} \frac{1+\kappa_{0}}{1+2 \kappa_{0}}+\tilde{\alpha}_{2}\left(-2 s \frac{1}{1+2 \kappa_{0}}+4 s(1-s) \frac{\left(1+\kappa_{0}\right)^{2}}{\left(1+2 \kappa_{0}\right)^{2}}\right)-\frac{\tilde{\alpha}_{2}}{1+2 \kappa_{0}} V\left(r_{0}\right)\right) .
\end{aligned}
$$

Finally note the relationship between the outgoing and ingoing solutions:

$$
\mathcal{R}^{\text {out },(s=2),(m)}(r)=\frac{4 \kappa^{2}}{r^{2}(r+2 \kappa)^{2}} \mathcal{R}^{\text {in },(s=-2),(-m)}(r) .
$$

\section{Emission from generic orbits from conformal trans- formations}

We now derive the gravitational wave emission from all other equatorial (corotating) orbits in (near-)NHEK. To do so we apply the conformal transformations described in Section 3 to relate the waveforms associated with generic equatorial orbits in (near-)NHEK to one of the solutions in the two sets of circular "seed orbits" given in Section 5 above.

In 29 31 this procedure was employed for particular orbits, and in 30,31 the analysis was limited to spin 0 probes. Here we generalize these considerations to gravitational wave emission from all equatorial orbits. An important technical subtlety, which we discuss in detail below, arises from the fact that conformal transformations do not in general conserve the form of the tetrad. Instead, the transformations must be accompanied by a Type III frame rotation that transforms the Weyl scalar as in A.16). Remarkably, however, we find that the conformal maps do preserve the nature of the boundary conditions at infinity and at the horizon: Dirichlet solutions remain Dirichlet, and ingoing solutions remain ingoing. This reduces the problem of finding the gravitational wave emission in the frequency domain of a plunging or osculating orbit to solving a particular integral over the real line, which arises from the Fourier transform of the conformal map at the boundary of (near)NHEK spacetime. Quite importantly, this Fourier integral can be analytically solved 
since it reduces for each orbit to an integral representation of an hypergeometric function or a simpler function. This fact directly originates from conformal symmetry.

There are four categories of conformal transformations mapping either (near-)NHEK into (near-)NHEK. Below we first derive the main formulae in a notation that is adapted to the case of a near-NHEK circular seed mapped to a NHEK orbit. In particular we use the convention that the final near-horizon coordinates of the physical solution are barred while the coordinates of the seed solution are unbarred, as in Appendix B.3. This notation will be easily adapted when we treat the remaining three cases in the rest of this Section, by switching lower and upper cases and updating some formulae while keeping all barred quantities barred.

\subsection{Circular near-NHEK orbit to NHEK orbits}

Let us first discuss the maps from the near-NHEK circular orbit, whose gravitational wave emission was just computed in Section 5, to NHEK orbits. There are two classes of such conformal maps, as shown in Section B.3.3. In order to find the gravitational waveform in the asymptotically flat region we must determine the coefficient $B$ in the asymptotic solution (4.23) of the Newman-Penrose scalar $\delta \psi_{4}$. This coefficient has its origin as the coefficient multiplying the Dirichlet solution of $\delta \psi_{4}$ in the near-horizon region. The near-NHEK circular seed solution $\delta \psi_{4}^{\text {circ }}(t, r, \theta, \phi)$ is given in (5.1)-(5.12). We denote the physical plunging/osculating solution in NHEK by $\delta \psi_{4}^{\text {physical }}(T, R, \theta, \Phi)$. The conformal maps relating both orbits reads as

$$
\bar{R}=\bar{R}(t, r), \quad \bar{T}=\bar{T}(t, r), \quad \bar{\Phi}=\phi+\delta \bar{\Phi}(t, r) .
$$

In general, this change of coordinates needs to be accompanied by a Type III frame rotation, $l_{\text {physical }}^{\mu}=F^{-1} l_{\text {seed }}^{\mu}, n_{\text {physical }}^{\mu}=F n_{\text {seed }}^{\mu}$, with $F=F(\bar{T}, \bar{R})$. The Newman-Penrose scalars are then related as

$$
\psi_{4}^{\text {physical }}(\bar{T}, \bar{R}, \theta, \bar{\Phi})=F^{2}(\bar{T}, \bar{R}) \psi_{4}^{\text {circ }}(t, r, \theta, \phi)
$$

In order to have enough freedom to enforce the two boundary conditions on the physical orbits, we allow to supplement the seed circular solution 5.12 with an additional homogeneous solution, i.e. we consider

$$
\begin{aligned}
R_{l m \tilde{\omega}}(r)= & \frac{\left(r_{0}\left(r_{0}+2 \kappa\right)\right)^{s}}{\tilde{W}}\left(\tilde{\mathcal{X}} \Theta\left(r_{0}-r\right) \mathcal{R}^{\mathrm{in}}(r)+\tilde{\mathcal{Z}} \Theta\left(r-r_{0}\right) \mathcal{R}^{\mathrm{D}}(r)+\tilde{\mathcal{Y}} \mathcal{R}^{\mathrm{in}}(r)\right) \\
& +\frac{\tilde{a}_{2}}{1+2 \kappa_{0}} \delta\left(r-r_{0}\right)
\end{aligned}
$$

where $\tilde{\mathcal{Y}}$ is not fixed. 
After a Fourier transformation, we obtain that the seed and physical solutions within the near-horizon region are related as

$$
R_{l m \Omega}(\bar{R})=\frac{1}{\sqrt{2 \pi}} \int_{-\infty}^{\infty} d \bar{T} e^{i \Omega \bar{T}-i m \tilde{\omega} t(\bar{T}, \bar{R})-i m \delta \bar{\Phi}(\bar{T}, \bar{R})} F^{2}(\bar{T}, \bar{R}) R_{l m \tilde{\omega}}(r(\bar{T}, \bar{R})) .
$$

All conformal transformations written in Appendix B.3 have the property that $\bar{R} \rightarrow \infty$ at fixed $\bar{T}$ is equivalent to $r \rightarrow \infty$ at fixed $t$, and that $\bar{R}=F(\bar{T}) r$, and $t=t(\bar{T})$ in the $\bar{R} \rightarrow \infty$ limit for a specific function $F$. Also, $\lim _{r \rightarrow \infty} F(\bar{T}, \bar{R})=F(\bar{T})$ for the same function $F$ and $\lim _{r \rightarrow \infty} \delta \bar{\Phi}(r, t)=0$. In the matching region we therefore have

$$
\lim _{\bar{R} \rightarrow \infty} R_{l m \Omega}(\bar{R})=\frac{1}{\sqrt{2 \pi}} \int_{-\infty}^{\infty} d \bar{T} e^{i(\Omega \bar{T}-m \tilde{\omega} t(\bar{T}))} F^{2}(\bar{T}) \lim _{r \rightarrow \infty} R_{l m \tilde{\omega}}(r) .
$$

This equation provides in particular the explicit map between homogeneous solutions. It shows that a Dirichlet mode is mapped to a Dirichlet mode and a Neumann mode is mapped to a Neumann mode because the two modes are proportional to each other in the asymptotic region. Considering the full solution, the transfer matrix from (6.5) is

$$
\left[\begin{array}{cc}
(-2 i \Omega)^{h} & c_{h}^{\Omega, m} \\
0 & c_{1-h}^{\Omega, m}
\end{array}\right]\left[\begin{array}{c}
B \\
A
\end{array}\right]=\frac{r_{0}^{s}\left(r_{0}+2 \kappa\right)^{s}}{\tilde{W}}\left[\begin{array}{cc}
T_{h}^{\Omega, m \tilde{\omega}} & T_{h}^{\Omega, m \tilde{\omega}} d_{h}^{n, m} \\
0 & T_{1-h}^{\Omega, m \tilde{\omega}} d_{1-h}^{n, m}
\end{array}\right]\left[\begin{array}{c}
\tilde{\mathcal{Z}} \\
\tilde{\mathcal{Y}}
\end{array}\right]
$$

where $c_{h}^{\Omega, m}$ and $d_{h}^{n, m}$ are defined respectively in 4.18) and 4.32 and the time integral can be absorbed into the coefficient

$$
T_{h}^{\Omega, m \tilde{\omega}}=\frac{1}{\sqrt{2 \pi}} \int_{-\infty}^{\infty} d \bar{T} e^{i(\Omega \bar{T}-m \tilde{\omega} t(\bar{T}))} F^{h+2+s}(\bar{T}) .
$$

Now, we also have that a purely ingoing mode solution is mapped to a purely ingoing mode solution. This follows from the fact that if there is no outgoing mode from the past black hole horizon then there will be no outgoing mode from the Poincaré horizon by continuity of the solutions to the wave equations. This argument is independent of the details of the conformal map. It implies that the ratio of Neumann to Dirichlet modes which characterizes an ingoing solution is preserved under the conformal map. In other words,

$$
\frac{c_{h}^{\Omega, m}}{c_{1-h}^{\Omega, m}}=\frac{T_{h}^{\Omega, m \tilde{\omega}} d_{h}^{n, m}}{T_{1-h}^{\Omega, m \tilde{\omega}} d_{1-h}^{n, m}}
$$

The final solution for the gravitational wave emission is thus given in 4.23 with $B$ given 
by (6.6). This yields $\AA^{12}$

$$
B=(-2 i \Omega)^{-h} \frac{r_{0}^{s}\left(r_{0}+2 \kappa\right)^{s}}{\tilde{W}} T_{h}^{\Omega, m \tilde{\omega}} \tilde{\mathcal{Z}}
$$

We now present the explicit emission formulae for the following two classes of orbits: $\operatorname{Marginal}(\ell)$ and Plunging $(E, \ell) / \operatorname{Osculating}(E, \ell)$.

Marginal $(\ell)$ As described in Appendix B.3.3, the conformal map in this case consists of the transformation (B.20) with final barred coordinates $(\bar{T}, \bar{R}, \bar{\Phi})$, combined with a PT flip $\bar{T} \mapsto-\bar{T}, \bar{\Phi} \mapsto-\bar{\Phi}$. Ignoring first the PT flip, a type III tetrad rotation is required with

$$
F(\bar{T}, \bar{R})=\frac{\bar{R}}{r(\bar{T}, \bar{R})}=-\frac{1}{\kappa \bar{T}}+O\left(\bar{R}^{-1}\right)
$$

where the asymptotic limit of the map is $(\bar{R} \rightarrow \infty$ with $\bar{T}, \bar{\Phi}$ fixed $)$

$$
\begin{aligned}
r & =-\kappa \bar{R} \bar{T}\left(1+O\left(\bar{R}^{-1}\right)\right), \\
t & =-\frac{1}{\kappa} \log |\bar{T}|+O\left(\bar{R}^{-1}\right), \\
\phi & =\bar{\Phi}+O\left(\bar{R}^{-1}\right) .
\end{aligned}
$$

Now, the PT flip $\bar{T} \mapsto-\bar{T}, \bar{\Phi} \mapsto-\bar{\Phi}$ can be taken into account by flipping the sign of both the angular momentum $m$ and frequency $\Omega$ of the NHEK solution. Since the frequency of the near-NHEK solution is $m \tilde{\omega}$, it will be automatically flipped as well. The angular equations between the near-NHEK and NHEK solutions however would not match since $S_{l, m}(\theta) \neq S_{l,-m}(\theta)$. Instead, the NHEK solution with $\theta$ angle has to be matched with the near-NHEK solution with $\pi-\theta$ angle. In the equatorial plane, the solutions and physical orbits are simply identified. The radial solutions can then be matched term by term in $l, m$ thanks to the identity $S_{l, m}(\theta)=S_{l,-m}(\pi-\theta)$. No tetrad rotation needs to be performed. One simply formally maps the $\theta$ NHEK solution to the $\pi-\theta$ near-NHEK solution with frequencies and angular momentum flipped.

The final $B$ coefficient is therefore (here $s=-2$ )

$$
B=(-2 i \Omega)^{-h} \frac{\tilde{\mathcal{Z}}}{r_{0}^{2}\left(r_{0}+2 \kappa\right)^{2} \tilde{W}} T_{h}^{-\Omega,-m \tilde{\omega}}
$$

\footnotetext{
${ }^{12}$ Arguably a simpler way to get the final result is to start from a Dirichlet solution in the matching region, perform the conformal map, and finally add an ingoing solution in order to obey the correct boundary condition with respect to the asymptotically flat region. Since the final step can always be performed and does not change the coefficient $B$, it can be ignored in the computation of $B$. The coefficient $B$ is obtained from the Dirichlet solution in the matching region: it is a function of $\tilde{\mathcal{Z}}$ only, and not of $\tilde{\mathcal{Y}}$, leading to 6.9 .
} 
where according to 6.7),

$$
T_{h}^{\Omega, m \tilde{\omega}}=\frac{1}{\sqrt{2 \pi}} \int_{-\infty}^{0} d \bar{T} e^{i \Omega \bar{T}}|\bar{T}|^{\frac{i m \tilde{\omega}}{\kappa}}(-\kappa \bar{T})^{-h} .
$$

Here the $\bar{T}$ integral is cut at $\bar{T}=0$ which is the endpoint of the trajectory corresponding to $t=-\infty$. Equivalently, in the notation adapted to the result of the PT flip,

$$
\begin{aligned}
T_{h}^{-\Omega,-m \tilde{\omega}} & =\frac{1}{\sqrt{2 \pi}} \int_{0}^{\infty} d \bar{T} e^{i \Omega \bar{T}} \bar{T}^{-\frac{i m \tilde{\omega}}{\kappa}-h} \kappa^{-h} \\
& =\frac{\kappa^{-h}}{\sqrt{2 \pi}}(-i \Omega)^{h-1+\frac{i m \tilde{\omega}}{\kappa}} \Gamma\left(1-h-\frac{i m \tilde{\omega}}{\kappa}\right)
\end{aligned}
$$

where the last integral is strictly valid for modes $h=\frac{1}{2}+i h_{I}, h_{I}<0$, which are the dominant modes as shown below.

As a cross-check, we can also understand the conformal map followed by the PT flip as a single map from the circular near-NHEK trajectory with outgoing boundary conditions at the horizon to the NHEK Marginal $(\ell)$ orbit with the same $\theta$ angle and same angular momentum $m$. The transformation relating the orbits is given by

$$
\begin{aligned}
r & =\kappa \bar{R} \bar{T}\left(1+O\left(\bar{R}^{-1}\right)\right) \\
t & =-\frac{1}{\kappa} \log \bar{T}+O\left(\bar{R}^{-1}\right) \\
\phi & =-\bar{\Phi}+O\left(\bar{R}^{-1}\right)
\end{aligned}
$$

Such a transformation transforms the tetrad frame as

$$
l^{\mu} \rightarrow \frac{2 M^{2}\left(1+\cos ^{2} \theta\right)}{-r R} N^{\mu}, n^{\mu} \rightarrow \frac{-r R}{2 M^{2}\left(1+\cos ^{2} \theta\right)} L^{\mu}, m^{\mu} \rightarrow \frac{1-i \cos \theta}{1+i \cos \theta} \bar{M}^{\mu} .
$$

After some algebra we find that the $s=-2$ waveform in NHEK is related to the $s=+2$ waveform in near-NHEK as

$$
\left.\psi_{(-2)}\right|_{N H E K}(\bar{R})=\left.\frac{r^{2} \bar{R}^{2}}{4 M^{4}} \psi_{(2)}\right|_{\text {near-NHEK }}(r) .
$$

This solution with outgoing boundary conditions at the horizon was computed in (5.2)5.14. We can now use the identity $S_{l, m}^{(2)}(\theta)=S_{l,-m}^{(-2)}(\theta)$ to relate the angular part of the $s=+2$ and $s=-2$ solutions. The radial NHEK solution is therefore obtained from the near-NHEK radial solution with $m \mapsto-m$. After some algebra, we find that $B$ can be written as

$$
B=\left.\frac{1}{4 M^{4}}(-2 i \Omega)^{-h} \frac{\left(r_{0}\left(r_{0}+2 \kappa\right)\right)^{2} \tilde{\mathcal{Z}}^{\prime}}{\tilde{W}^{\prime}}\right|_{m \mapsto-m} T_{h}^{-\Omega,-m \tilde{\omega}}
$$


where $\tilde{W}^{\prime}$ and $\tilde{\mathcal{Z}}^{\prime}$ are defined in $(5.16)$. We checked that $(6.14)$ and $(6.23)$ identically agree after using the property (5.17). This provides a non-trivial cross-check of our formulæ.

The final gravitational wave flux (4.23) is

$$
\begin{aligned}
\delta \psi_{4}(\hat{r} \rightarrow \infty) & =\frac{M^{2}}{2 \pi} \int_{-\infty}^{\infty} \mathrm{d} \Omega \sum_{l m} \frac{\tilde{\mathcal{Z}}(2 \kappa)^{-h}}{\tilde{W}\left(r_{0}\left(r_{0}+2 \kappa\right)\right)^{2}} \mathcal{K}(-i \Omega)^{-1+\frac{i m \tilde{\omega}}{\kappa}} \\
& \times \Gamma\left(1-h-\frac{i m \tilde{\omega}}{\kappa}\right) S_{l m}(\theta) e^{i m \hat{\phi}} e^{-i \frac{m+\lambda^{2 / 3} \Omega}{2 M}} \hat{u}^{-1} .
\end{aligned}
$$

Effectively, the integral should be cut above $\Omega \sim \lambda^{-2 / 3}$ in order to remain in the domain of validity of the asymptotically matched expansion scheme. In order to find the large $\hat{u}$ behavior of the waveform, we require that $\lambda^{2 / 3} \frac{\hat{u}}{2 M}$ is finite in the limit $\lambda \rightarrow 0, \hat{u} \rightarrow \infty$. Indeed, the NHEK orbit is only valid for a finite range of retarded time $\Delta \hat{u}$ which we expect by scaling to be $\Delta \hat{u} \sim M \lambda^{-2 / 3}$. Defining the new integration variable

$$
\Phi \equiv \hat{\omega} \hat{u}-\frac{m}{2 M} \hat{u}=\frac{\lambda^{2 / 3} \hat{u}}{2 M} \Omega
$$

and using (4.24), the large $\hat{u}$ behavior of the waveform becomes

$$
\delta \psi_{4}(\hat{r} \rightarrow \infty)=\sum_{l m} \frac{M^{2} \tilde{\mathcal{Z}} \kappa^{-h}}{\tilde{W}\left(r_{0}\left(r_{0}+2 \kappa\right)\right)^{2}} S_{l m}(\theta) e^{i m\left(\hat{\phi}-\frac{\hat{u}}{2 M}\right)} \hat{x}^{-1} \lambda^{-\frac{2 i m \tilde{\omega}}{3 \kappa}} k_{1}\left(\frac{\hat{u}}{2 M}\right)^{-h-\frac{i m \tilde{\omega}}{\kappa}} I^{l, m, \tilde{\omega}}
$$

where the remaining integral is

$$
I^{l, m, \tilde{\omega}}=\frac{\Gamma\left(1-h-\frac{i m \tilde{\omega}}{\kappa}\right)}{2 \pi} \lim _{\hat{u} \rightarrow \infty} \int_{-\frac{\hat{u}}{2 M}}^{\frac{\hat{u}}{2 M}} d \Phi \frac{(-i \Phi)^{h-1+\frac{i m \tilde{\omega}}{\kappa}} e^{-i \Phi}}{1-\left(-i \Phi \frac{2 M}{\hat{u}}\right)^{2 h-1} k_{2}}
$$

To find the large time behavior, we should distinguish two subcases:

- $\operatorname{Re}(h)>\frac{1}{2}$. We can then neglect the denominator inside the integrand and recognize the integral as an inverse Laplace transform [48, 3.381, p348

$$
\frac{1}{2 \pi i} \int_{-i \infty+\epsilon}^{i \infty+\epsilon} d s s^{h-1+\frac{i m \tilde{\omega}}{\kappa}} e^{s}=\frac{1}{\Gamma\left(1-h-\frac{i m \tilde{\omega}}{\kappa}\right)} .
$$

The waveform is then

$$
\delta \psi_{4}(\hat{r} \rightarrow \infty)=\sum_{l m} \frac{M^{2} \tilde{\mathcal{Z}} \kappa^{-h}}{\tilde{W}\left(r_{0}\left(r_{0}+2 \kappa\right)\right)^{2}} S_{l m}(\theta) \frac{e^{i m\left(\hat{\phi}-\frac{\hat{u}}{2 M}\right)}}{\hat{x}} \frac{k_{1}}{\lambda^{\frac{2 i m \tilde{\omega}}{3 \kappa}}}\left(\frac{\hat{u}}{2 M}\right)^{-h-\frac{i m \tilde{\omega}}{\kappa}}(6.2
$$

and $\left|\delta \psi_{4}\right| \propto\left(\frac{\hat{u}}{2 M}\right)^{-\operatorname{Re}(h)}$ in this regime.

\footnotetext{
${ }^{13}$ The $\epsilon$ prescription with $\epsilon>0, \epsilon \rightarrow 0$ is required to make the integral well-defined. This defines our prescription for avoiding the branch cut. We assume that this regulator originates from subleading correction in the $\lambda \rightarrow 0$ limit.
} 
- $\operatorname{Re}(h)=\frac{1}{2}$. The integral factor will oscillate as $\hat{u}$ increases. Now, for $h=\frac{1}{2}+i h_{I}$ and $h_{I}<0$

$$
\left|k_{2}\right|=e^{\pi h_{I}} \frac{\cosh \pi\left(m+h_{I}\right)}{\cosh \pi\left(m-h_{I}\right)}<10^{-3}
$$

for all modes $l \geq 2, m>0$. In particular, the modes $(l, m)=(9,7)$ and $(l, m)=$ $(13,10)$ have $\left|k_{2}\right| \approx 10^{-4}$. We numerically checked for those cases that $I^{l, m, \tilde{\omega}} \approx 1$ up to a small $\left(\approx 10^{-3}\right)$ variation. We deduce that $\left|\delta \psi_{4}\right| \propto\left(\frac{\hat{u}}{2 M}\right)^{-\operatorname{Re}(h)}$.

Since we assumed $\lambda^{2 / 3} \frac{\hat{u}}{2 M}$ finite we obtain in both cases

$$
\left|\delta \psi_{4}\right| \propto \lambda^{0}\left(\frac{\hat{u}}{2 M}\right)^{-\operatorname{Re}(h)} \propto \mathcal{O}\left(\lambda^{\frac{2}{3} \operatorname{Re}(h)}\right)
$$

which is similar to the scaling behavior of the circular NHEK orbit. The modes with the leading amplitude in the near-extremal limit are therefore the modes with $\operatorname{Re}(h)=\frac{1}{2}$ and they fall-off as $1 / \sqrt{\hat{u}} .^{14}$ As an illustration, the time evolution of $\left|\delta \psi_{4}(\hat{r} \rightarrow \infty)\right|$ (normalized to unity at $\hat{u}=1)$ is shown for three different modes with $\operatorname{Re}(h)=\frac{1}{2}$ in Figure 4 .

Plunging $(E, \ell) /$ Osculating $(E, \ell)$ The transformation 2.10 followed by a PT flip $\Phi \mapsto$ $-\Phi, T \mapsto-T$ followed by (B.34), which connects a Plunging $(E, \ell)$ orbit with a nearNHEK circular orbit requires the following frame transformation

$$
\begin{aligned}
l^{\mu} & \rightarrow \frac{-2 M^{2}\left(1+\cos ^{2} \theta\right)}{r \bar{R}} \frac{1+\bar{R} \bar{T}+\bar{R} \cot \frac{\zeta}{2}}{-1+\bar{R} \bar{T}+\bar{R} \cot \frac{\zeta}{2}} N^{\mu}, \\
n^{\mu} & \rightarrow \frac{r \bar{R}}{-2 M^{2}\left(1+\cos ^{2} \theta\right)} \frac{-1+\bar{R} \bar{T}+\bar{R} \cot \frac{\zeta}{2}}{1+\bar{R} \bar{T}+\bar{R} \cot \frac{\zeta}{2}} L^{\mu}, \\
m^{\mu} & \rightarrow \frac{1-i \cos \theta}{1+i \cos \theta} \bar{M}^{\mu}
\end{aligned}
$$

where $\zeta$ is related to the orbit parameters as $(\mathrm{B} .35)$. It implies that

$$
\left.\psi_{(-2)}\right|_{N H E K}(\bar{R})=\left.\frac{r^{2} \bar{R}^{2}}{4 M^{4}}\left(\frac{-1+\bar{R} \bar{T}+\bar{R} \cot \frac{\zeta}{2}}{1+\bar{R} \bar{T}+\bar{R} \cot \frac{\zeta}{2}}\right)^{2} \psi_{(2)}\right|_{\text {near }-N H E K}(r) .
$$

The coordinate transformation is given asymptotically by

$$
\begin{aligned}
r & =\kappa \bar{R}\left(\bar{T} \cos \zeta-\frac{1}{2}\left(1-\bar{T}^{2}\right) \sin \zeta\right)\left(1+O\left(\bar{R}^{-1}\right)\right) \\
t & =-\frac{1}{\kappa} \log \frac{\bar{T} \cos \zeta-\frac{1}{2}\left(1-\bar{T}^{2}\right) \sin \zeta}{\left(\cos \frac{\zeta}{2}+\sin \frac{\zeta}{2} \bar{T}\right)^{2}}\left(1+O\left(\bar{R}^{-1}\right)\right) \\
\phi & =-\bar{\Phi}\left(1+O\left(\bar{R}^{-1}\right)\right)
\end{aligned}
$$

\footnotetext{
${ }^{14}$ This result is in tension with a recent numerical simulation 47 . which claims to obtain a $1 / \hat{u}$ behavior for one example of Marginal( $\ell$ ) orbit (in our terminology).
} 

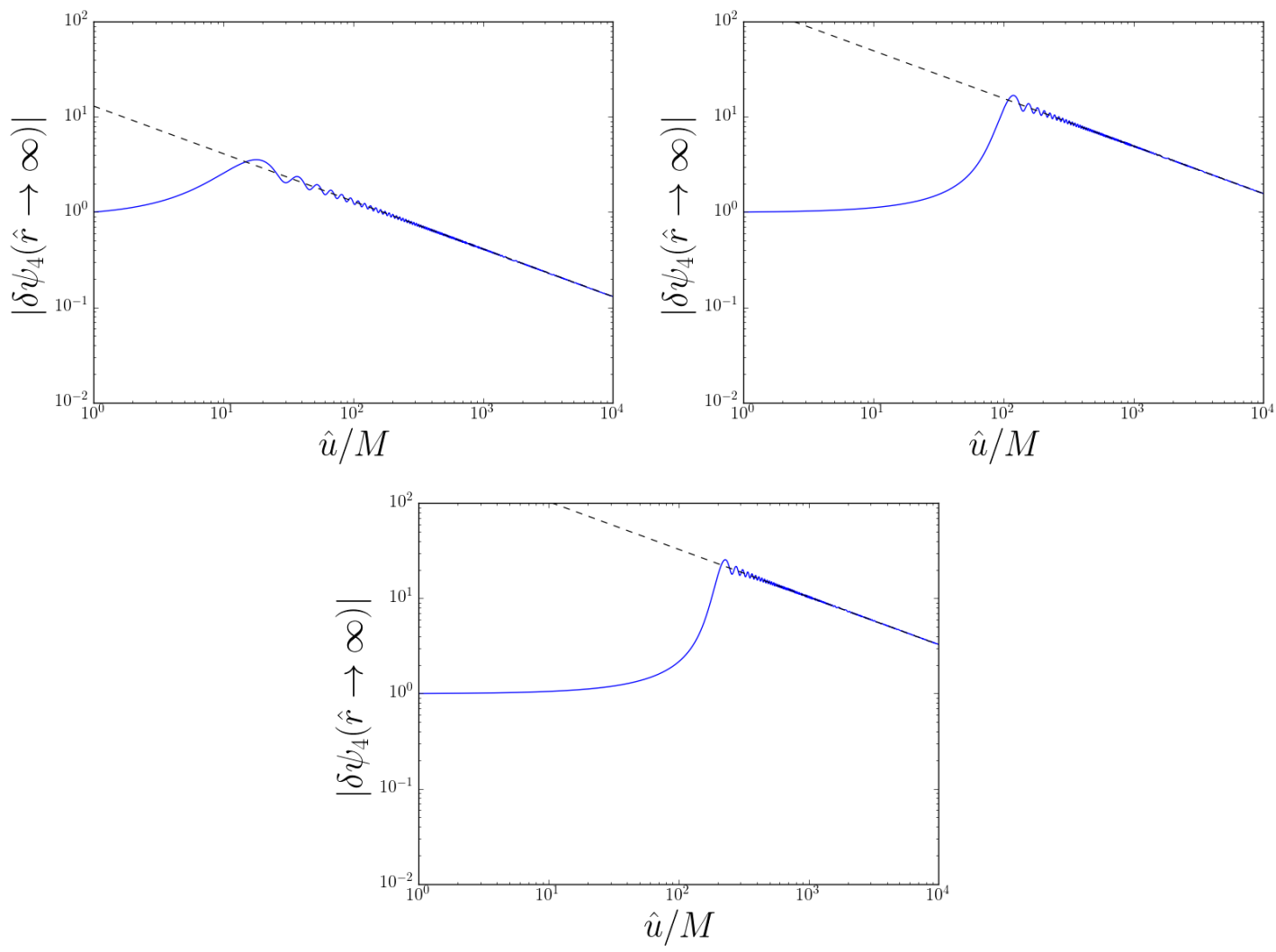

Figure 4: Envelope time-evolution of $\delta \psi_{4}(\hat{r} \rightarrow \infty)$ (normalized) for the Marginal( $(\ell)$ orbits of the modes (top-left) $l=2, m=2$; (top-right) $l=50, m=38$ and (bottom) $l=50, m=50$ with $\lambda=10^{-3}$ and $\ell=2 \ell_{*}$. The late time behavior is given by $\left(\frac{\hat{u}}{2 M}\right)^{-1 / 2}$ as indicated by the dashed line.

The trajectory ends at $\bar{T} \rightarrow+\infty$ and starts at $t \rightarrow+\infty$ (because of the PT flip) which is $\bar{T}=\tan \frac{\zeta}{2}$. forms

Repeating the same analysis as in the Marginal $(\ell)$ case, one finds the two equivalent

$$
\begin{aligned}
B_{l m \Omega} & =\left.\frac{(-2 i \kappa \Omega)^{-h}}{\sqrt{2 \pi}} \frac{r_{0}^{2}\left(r_{0}+2 \kappa\right)^{2} \tilde{\mathcal{Z}}^{\prime}}{4 M^{4} \tilde{W}^{\prime}}\right|_{m \mapsto-m, s=2} T_{h}^{\Omega, m \tilde{\omega}} \\
& =\left.\frac{(-2 i \kappa \Omega)^{-h}}{\sqrt{2 \pi}} \frac{\tilde{\mathcal{Z}}}{r_{0}^{2}\left(r_{0}+2 \kappa\right)^{2} \tilde{W}}\right|_{s=-2} T_{h}^{\Omega, m \tilde{\omega}}
\end{aligned}
$$


with

$$
\begin{aligned}
T_{h}^{\Omega, m \tilde{\omega}} & =\int_{\tan \frac{\zeta}{2}}^{\infty} d \bar{T} e^{i \Omega \bar{T}}\left(\bar{T} \cos \zeta-\frac{1}{2}\left(1-\bar{T}^{2}\right) \sin \zeta\right)^{-h-\frac{i m \tilde{\omega}}{\kappa}}\left(\bar{T} \sin \frac{\zeta}{2}+\cos \frac{\zeta}{2}\right)^{\frac{2 i m \tilde{\omega}}{\kappa}} \\
& =\left(\cot \frac{\zeta}{2}\right)^{-\frac{i m \tilde{\omega}}{\kappa}} \Gamma\left(1-h-\frac{i m \tilde{\omega}}{\kappa}\right)(-i \Omega)^{h-1} e^{-i \Omega \cot \zeta} W_{\frac{i m \tilde{\omega}}{\kappa}, \frac{1}{2}-h}\left(\frac{-2 i \Omega}{\sin \zeta}\right)
\end{aligned}
$$

after using $(\mathrm{D} .9)$ with $p=-i \Omega$ and $y=\frac{i m \tilde{\omega}}{\kappa}$. The Weyl scalar in the asymptotically flat spacetime then follows from 4.23 and 4.24

$$
\begin{aligned}
\delta \psi_{4}(\hat{r} \rightarrow \infty) & =\frac{M^{2}}{2 \pi} \int_{-\infty}^{\infty} \mathrm{d} \Omega \sum_{l m} \frac{\tilde{\mathcal{Z}}(2 \kappa)^{-h}}{\tilde{W}\left(r_{0}\left(r_{0}+2 \kappa\right)\right)^{2}} S_{l m}(\theta) e^{i m \hat{\phi}} e^{-i \frac{m+\lambda^{2 / 3} \Omega}{2 M} \hat{u}} \\
& \times\left(\left(\cot \frac{\zeta}{2}\right)^{-\frac{i m \tilde{\omega}}{\kappa}}(-i \Omega)^{-1} \mathcal{K} e^{-i \Omega \cot \zeta} \Gamma\left(1-h-\frac{i m \tilde{\omega}}{\kappa}\right) W_{\frac{i m \tilde{\omega}}{\kappa}, \frac{1}{2}-h}\left(\frac{-2 i \Omega}{\sin \zeta}\right)\right) \hat{x}^{-1} \\
& =\sum_{l m} \frac{M^{2} \tilde{\mathcal{Z}} \kappa^{-h}}{\tilde{W}\left(r_{0}\left(r_{0}+2 \kappa\right)\right)^{2}} S_{l m}(\theta) \frac{e^{i m \hat{\phi}-i \frac{m}{2 M} \hat{u}}}{\hat{x}} k_{1}\left(\frac{\hat{u}}{2 M}\right)^{-h} I_{\hat{u}, \lambda}^{l, m} .
\end{aligned}
$$

The integral can be expressed as an inverse Laplace transform and it can be evaluated explicitly in a similar approximation as in the Marginal $(\ell)$ case using [48, 7.522, p823]

$$
\begin{aligned}
I_{\hat{u}, \lambda}^{l, m} & =\frac{\Gamma\left(1-h-\frac{i m \tilde{\omega}}{\kappa}\right)}{2 \pi\left(\cot \frac{\zeta}{2}\right)^{\frac{i m \tilde{\omega}}{\kappa}}} \int_{-\infty}^{\infty} d \Phi \frac{(-i \Phi)^{h-1} e^{-i\left(1+\cot \zeta \frac{2 M}{\hat{u} \lambda^{2 / 3}}\right) \Phi}}{1-(-2 i M \Phi / \hat{u})^{2 h-1} k_{2}} W_{\frac{i m \tilde{\omega}}{\kappa}, \frac{1}{2}-h}\left(\frac{-4 i M \Phi}{\sin \zeta \hat{u} \lambda^{2 / 3}}\right) \\
& \approx \frac{\Gamma\left(1-h-\frac{i m \tilde{\omega}}{\kappa}\right)}{\left(\cot \frac{\zeta}{2}\right)^{\frac{i m \tilde{\omega}}{\kappa}} 2 \pi i} \int_{-i \infty+\epsilon}^{i \infty+\epsilon} \mathrm{d} s e^{s\left(1+\cot \zeta \frac{2 M}{\hat{u} \lambda^{2 / 3}}\right)} s^{h-1} W_{\frac{i m \tilde{\omega}}{\kappa}, \frac{1}{2}-h}\left(\frac{4 M}{\sin \zeta \hat{u} \lambda^{2 / 3}} s\right) \\
& =\left(\frac{2 M}{\cos ^{2} \frac{\zeta}{2} \hat{u} \lambda^{2 / 3}}\right)^{\frac{i m \tilde{\omega}}{\kappa}}\left(1-\frac{2 M}{\hat{u} \lambda^{2 / 3}} \tan \frac{\zeta}{2}\right)^{-h-\frac{i m \tilde{\omega}}{\kappa}}\left(1+\frac{\sin \zeta \hat{u} \lambda^{2 / 3}}{4 M}\left(1-\frac{2 M}{\hat{u} \lambda^{2 / 3}} \tan \frac{\zeta}{2}\right)\right)^{-h+\frac{i m \tilde{\omega}}{\kappa}} .
\end{aligned}
$$

The auxiliary parameters $\left(\zeta, T_{0}\right)$ can be traded for the physical NHEK energy $E$ and the initial NHEK time impact parameter $\bar{T}_{0}$ via $(B .35)$, which we rewrite for convenience:

$$
E=\frac{\sqrt{3\left(\ell^{2}-\ell_{*}^{2}\right)}}{2}\left(\sin \zeta+T_{0}(\cos \zeta-1)\right), \quad \bar{T}_{0}=\frac{-\cos \zeta+T_{0} \sin \zeta}{\sin \zeta+T_{0}(\cos \zeta-1)}
$$

If $\zeta=0$, or equivalently, $E=0, \bar{T}_{0} \rightarrow-\infty$, this matches the result 6.29 of the $\operatorname{Marginal}(\ell)$ case valid for any $\bar{T}_{0}$, which is a consistency check. In that case $\left|\delta \psi_{4}\right| \propto$ $\left(\frac{\hat{u}}{2 M}\right)^{-\operatorname{Re}(h)}$. For more general energies, the integral contributes an additional factor $\left(\frac{\hat{u} \lambda^{2 / 3}}{2 M}\right)^{-h}$ at large $\hat{u} \lambda^{2 / 3}$ such that $\left|\delta \psi_{4}\right| \propto\left(\frac{\hat{u}}{2 M}\right)^{-2 \operatorname{Re}(h)}$. This is illustrated in Figure 5 for $E \approx 0.1 \frac{3 \ell_{*}}{2}$ and $E=\frac{3 \ell_{*}}{4}$. The interpolation between the $\hat{u}^{-h}$ and $\hat{u}^{-2 h}$ polynomial behaviors depending on the source parameters is reminiscent of the near-NHEK signal 

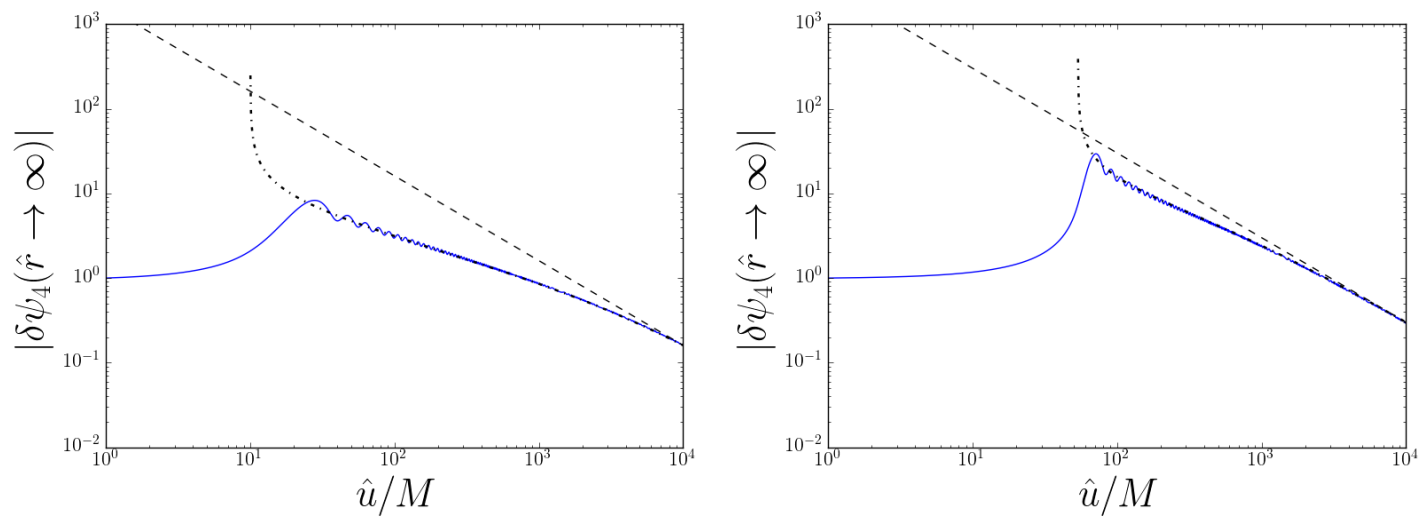

Figure 5: Envelope time-evolution of the (normalized) $l=2, m=2$ mode $\delta \psi_{4}(\hat{r} \rightarrow$ $\infty)$ for the Plunging $(E, \ell)$ orbits with (left) $E \approx 0.1 \frac{3 \ell_{*}}{2}$ and (right) $E=$ $\frac{3 \ell_{*}}{4}$, both with $\lambda=10^{-3}$ and $\ell=2 \ell_{*}$. The late time behavior is given by $\left(\frac{\hat{u}}{2 M}\right)^{-1}$ as indicated by the dashed line. The dotted-dashed line gives $\delta \psi_{4}(\hat{r} \rightarrow \infty)$ using the approximate analytical result for $I_{\hat{u}, \lambda}^{l, m}$.

during the polynomial ringdown phase as expressed in (4.47). This is also what will be seen explicitly for the Plunging $(e, \ell)$ orbits. The scaling with $\lambda$ is the same as for the Marginal $(\ell)$ orbits

$$
\left|\delta \psi_{4}\right| \propto \mathcal{O}\left(\lambda^{\frac{2}{3} \operatorname{Re}(h)}\right)
$$

\subsection{Circular NHEK orbit to NHEK orbits}

We are aimed at finding $B$ in 4.23 which is determined from the near-horizon physics. The NHEK circular solution is written in (C.3), C.14). Since there is only one class of orbits conformally related to the ISCO, namely the Plunging ${ }_{*}(E)$ orbit, we will make all formulae explicit.

Plunging $_{*}(E)$ This orbit is related to a circular NHEK orbit by (B.26) which requires an accompanying type III tetrad rotation with

$$
F=\frac{\bar{R}^{2}}{(1+\bar{R} \bar{T})^{2}}=\frac{1}{\bar{T}^{2}}+O\left(\bar{R}^{-1}\right)
$$

Asymptotically, the conformal map takes the form

$$
T=-\frac{1}{\bar{T}}, \quad R=\bar{R} \bar{T}^{2}, \quad \Phi=\bar{\Phi}
$$


Even though the final coordinates are barred, we will keep the final frequency $\Omega$ unbarred. The origin of the orbit $\bar{T}=0$ corresponds to $T=-\infty$. Combining these elements with the solution for the circular NHEK orbit results in

$$
\begin{aligned}
B & =\frac{(-2 i m \tilde{\Omega})^{h}}{(-2 i \Omega)^{h}} \frac{\mathcal{Z}}{W R_{0}^{4}} \frac{1}{\sqrt{2 \pi}} \int_{0}^{\infty} \mathrm{d} \bar{T} e^{i \Omega \bar{T}} e^{\frac{i m \tilde{\Omega}}{T}} \bar{T}^{-2 h} \\
& =\frac{2(-2 i m \tilde{\Omega})^{\frac{1}{2}}}{\sqrt{2 \pi}(-2 i \Omega)^{\frac{1}{2}}} \frac{\mathcal{Z}}{W R_{0}^{4}} K_{1-2 h}(2 \sqrt{-m \Omega \tilde{\Omega}})
\end{aligned}
$$

after using $(\mathrm{D} .13)$ with $p=-i \Omega$ and $y=-i m \tilde{\Omega}^{15}$. The asymptotic curvature perturbation (4.23) reads as

$$
\begin{aligned}
\delta \psi_{4}(\hat{r} \rightarrow \infty) & =\frac{M^{2}}{\pi \hat{x}} \int_{-\infty}^{\infty} \mathrm{d} \Omega \sum_{l m} \frac{(-2 i m \tilde{\Omega})^{\frac{1}{2}}}{(-2 i \Omega)^{\frac{1}{2}}} \frac{\mathcal{Z} \mathcal{K}}{W R_{0}^{4}} K_{1-2 h}(2 \sqrt{-m \Omega \tilde{\Omega}}) S_{l m}(\theta) e^{i m \hat{\phi}} e^{-i \frac{m+\lambda^{2 / 3} \Omega}{2 M} \hat{u}} \\
& =\frac{M^{2}}{\hat{x}} \sum_{l m} \lambda^{-1 / 3}\left(\frac{\hat{u}}{2 M}\right)^{-1 / 2-h}(-2 i m \tilde{\Omega})^{\frac{1}{2}} \frac{\mathcal{Z}(2)^{h-\frac{1}{2}} k_{1}}{W R_{0}^{4}} S_{l m}(\theta) e^{i m \hat{\phi}} e^{-i \frac{m}{2 M} \hat{u}} I_{\hat{u}, \lambda}^{l, m}(6.47)
\end{aligned}
$$

where $\mathcal{K}$ is defined in (4.24), $\mathcal{Z}, W$ in C.15), $\tilde{\Omega}$ in (C.2). Using (6.25) and keeping $\hat{u} \lambda^{2 / 3}$ fixed we have

$$
\begin{aligned}
I_{\hat{u}, \lambda}^{l, m} & \equiv \frac{1}{\pi} \int_{-\infty}^{\infty} d \Phi \frac{(-i \Phi)^{h-1 / 2}}{1-\left(-i \frac{2 M \Phi}{\hat{u}}\right)^{2 h-1} k_{2}} e^{-i \Phi} K_{1-2 h}\left(2 \sqrt{-m \tilde{\Omega} \Phi \frac{2 M}{\hat{u} \lambda^{2 / 3}}}\right) \\
& \approx\left(-i m \tilde{\Omega} \frac{2 M}{\hat{u} \lambda^{2 / 3}}\right)^{h-1 / 2} e^{i m \tilde{\Omega} \frac{2 M}{\hat{u} \lambda^{2 / 3}}}
\end{aligned}
$$

where the second step approximates the integral by neglecting the denominator such that one can recognize it as the inverse Laplace transform of [48, 7.629, p836]. It follows that for Plunging ${ }_{*}(E)$ orbits the late time signal has an inverse time behavior. Performing the integral numerically leads to results which are qualitatively very similar to the previous cases. This is illustrated in Figure 6. In addition, for all NHEK orbits the amplitude of the signal is suppressed by the near-extremality factor $\lambda^{1 / 3}$.

\subsection{Circular NHEK orbit to near-NHEK orbits}

Let us first consider the near-NHEK orbits related via conformal map to the ISCO in the NHEK spacetime. As previously, we use the barred notation for the final coordinates where the orbit is described but keep $\omega$ unbarred. After mapping a generic near-NHEK

\footnotetext{
${ }^{15}$ We are considering a $+i \epsilon$ prescription in both frequencies to be able to use the explicit integral. We then set $\epsilon=0$.
} 


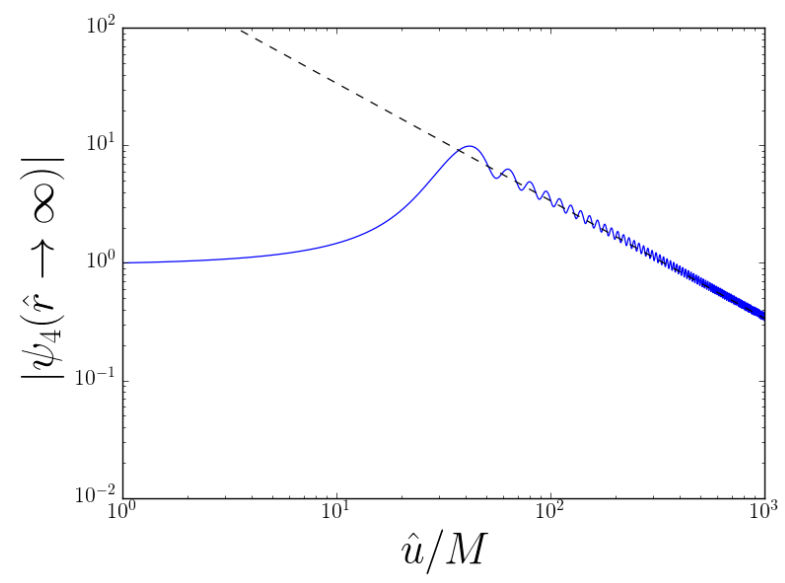

Figure 6: Envelope time-evolution of the (normalized) $l=2, m=2$ mode $\delta \psi_{4}(\hat{r} \rightarrow$ $\infty)$ for the Plunging ${ }_{*}(E)$ orbits with $\lambda=10^{-3}$ and $E=2 \ell_{*}$. The late time behavior is given by $\left(\frac{\hat{u}}{2 M}\right)^{-1}$ as indicated by the dashed line.

orbit of frequency $\omega$ to the ISCO orbit of frequency $\Omega=m \tilde{\Omega}$ and using 4.18 - 4.32 and (6.2), we find that the $B$ coefficient in 4.35 is given by

$$
B=\frac{\mathcal{Z}}{R_{0}^{4} W}(-2 i m \tilde{\Omega})^{h} \frac{1}{\sqrt{2 \pi}} \int_{\bar{t}_{-}}^{\bar{t}_{+}} \mathrm{d} \bar{t} F^{h}(\bar{t}) e^{i \omega \bar{t}-i m \tilde{\Omega} T(\bar{t})} .
$$

where $\bar{r}=F(\bar{t}) R$ is the leading order change of coordinate near the boundary $\bar{r} \rightarrow \infty$ and $\bar{t}_{ \pm}$are the initial and final times of the trajectory which are deduced from the change of coordinates from the ISCO.

There are 2 such classes of orbits: Plunging $(e=0)$ and $\operatorname{Plunging}_{*}(e)$ as described in Section B.3.2, which we consider sequentially in what follows.

Plunging $_{*}(e=0)$ In the limit $\bar{r} \rightarrow \infty$ with $\bar{t}$ and $\bar{\phi}$ fixed the transformation 2.10 relating a circular orbit in NHEK to a Plunging $(e=0)$ orbit $(B .8)$ becomes

$$
\begin{aligned}
R & =\frac{e^{\kappa \bar{t}}}{\kappa} \bar{r}\left(1+O\left(\bar{r}^{-1}\right)\right) \\
T & =-e^{-\kappa \bar{t}}+O\left(\bar{r}^{-1}\right) \\
\Phi & =\bar{\phi}+O\left(\bar{r}^{-1}\right) .
\end{aligned}
$$

The trajectory starts at $\bar{t} \rightarrow-\infty$ and ends at $\bar{t} \rightarrow+\infty$. The matching of NHEK A.22 and near-NHEK (A.25) tetrads requires a type III rotation with

$$
F(\bar{t})=\frac{\bar{r}}{R}=\kappa e^{-\kappa \bar{t}} .
$$


We find

$$
\begin{aligned}
B & =\frac{\mathcal{Z}}{R_{0}^{4} W}(-2 i m \tilde{\Omega})^{h} \frac{1}{\sqrt{2 \pi}} \int_{-\infty}^{\infty} \mathrm{d} \bar{t}\left(\kappa e^{-\kappa \bar{t}}\right)^{h} e^{i \omega \bar{t}+i m \tilde{\Omega} e^{-\kappa \bar{t}}} \\
& =\frac{1}{\sqrt{2 \pi}} 2^{h-i \frac{\omega}{\kappa}} \kappa^{h-1} \frac{\mathcal{Z}}{R_{0}^{4} W}(-2 i m \tilde{\Omega})^{i \frac{\omega}{\kappa}} \Gamma\left(h-i \frac{\omega}{\kappa}\right)
\end{aligned}
$$

after using the integral (D.1) in Appendix D. This now fixes the Weyl scalar in the asymptotically flat spacetime.

In order to obtain the late time behavior, we can make the quasi-normal mode approximation and the resulting Weyl scalar is 4.43$)$. The factor $\Gamma\left(h-i \frac{\omega}{\kappa}\right)$ exactly cancels $1 / \Gamma(h-i(n-m))$. The overtone sum can be performed explicitly using

$$
\sum_{N} \frac{(-1)^{N}}{N ! 2^{N}}(-2 i m \tilde{\Omega})^{N+h} e^{-i \frac{m-i \lambda(N+h)}{2 M} \hat{u}}=e^{-i \frac{m-i \lambda h}{2 M} \hat{u}+i m \tilde{\Omega} e^{-\frac{\lambda \hat{u}}{2 M}}}(-2 i m \tilde{\Omega})^{h} .
$$

The final answer in the quasi-normal mode approximation is

$$
\delta \psi_{4}(\hat{r} \rightarrow \infty)=\sum_{l, m} M^{2} \lambda^{h} k_{1} \frac{\mathcal{Z}}{R_{0}^{4} W}(-2 i m \tilde{\Omega})^{h} e^{-i \frac{m-i \lambda h}{2 M} \hat{u}+i m \tilde{\Omega} e^{-\frac{\lambda \hat{u}}{2 M}}} e^{i m \hat{\phi}} S_{l m}(\theta) \hat{x}^{-1} .
$$

The dominant late time behavior is now

$$
\left|\delta \psi_{4}(\hat{r} \rightarrow \infty)\right| \propto \lambda^{1 / 2} e^{-\frac{\lambda \hat{u}}{4 M}}
$$

Plunging $_{*}(\mathbf{e}) \quad$ Similarly, the Plunging ${ }_{*}(\mathrm{e})$ orbits $(\mathrm{B} .10)$ are in the same equivalence class as the NHEK circular orbits. It is described as (2.10) followed with $(\overline{B .22})$. The map of parameters is given in $\mathrm{B} .32$. For general $\zeta$, the transformation is

$$
\begin{aligned}
R & =-\frac{\bar{r}}{\kappa}(\cos \zeta-\sin \zeta \sinh \kappa \bar{t}) T^{-1}+o(\bar{r}) \\
T & =-\sqrt{\frac{\cosh \kappa \bar{t}-\cos \zeta \sinh \kappa \bar{t}-\sin \zeta}{\cosh \kappa \bar{t}+\cos \zeta \sinh \kappa \bar{t}+\sin \zeta}}+o\left(\bar{r}^{0}\right) \equiv T(\bar{t})+o\left(\bar{r}^{0}\right), \\
\Phi & =\bar{\phi}+o\left(\bar{r}^{0}\right)
\end{aligned}
$$

We find a type III rotation with

$$
\begin{aligned}
F(\bar{r}, \bar{t}) & =\frac{\sqrt{\bar{r}(\bar{r}+2 \kappa)}}{R} \frac{\sqrt{\bar{r}(\bar{r}+2 \kappa)} \sin \zeta \sinh \kappa \bar{t}-(\bar{r}+\kappa) \cos \zeta+\kappa}{(\bar{r}+\kappa) \sin \zeta \sinh \kappa \bar{t}-\sqrt{\bar{r}(\bar{r}+2 \kappa)} \cos \zeta+\kappa \sin \zeta \cosh \kappa \bar{t}}, \\
& =\frac{-\kappa T(\bar{t})}{\cos \zeta-\sin \zeta \sinh \kappa \bar{t}}+o\left(\bar{r}^{0}\right) .
\end{aligned}
$$


Then

$$
B=\frac{1}{\sqrt{2 \pi}} \kappa^{h}(-2 i m \tilde{\Omega})^{h} \frac{\mathcal{Z}}{R_{0}^{4} W} I_{\omega}^{l, m}
$$

where the remaining integral is

$$
\begin{aligned}
I_{\omega}^{l, m}= & \int_{\frac{\log \left(-\tan \frac{\zeta}{2}\right)}{\kappa}}^{\infty} \mathrm{d} \bar{t} e^{i \omega \bar{t}} e^{i m \tilde{\Omega} \sqrt{\frac{\cosh \kappa \bar{t}-\cos \zeta \sinh \kappa \bar{t}-\sin \zeta}{\cosh \kappa \bar{t}+\cos \zeta \sinh \kappa \bar{t} \sin \zeta}}} \\
& \times\left(\frac{1}{\cos \zeta-\sin \zeta \sinh \kappa \bar{t}} \sqrt{\frac{\cosh \kappa \bar{t}-\cos \zeta \sinh \kappa \bar{t}-\sin \zeta}{\cosh \kappa \bar{t}+\cos \zeta \sinh \kappa \bar{t}+\sin \zeta}}\right)^{h} \\
= & 2^{h} \kappa^{-1}\left(-\tan \frac{\zeta}{2}\right)^{\frac{i \omega}{\kappa}}(-\csc \zeta)^{h} e^{-i m \tilde{\Omega} \tan \frac{\zeta}{2}} \Gamma\left(h-i \frac{\omega}{\kappa}\right) U\left(h-i \frac{\omega}{\kappa}, 2 h, 2 i m \tilde{\Omega} \csc \zeta\right) \\
= & 2^{h} \kappa^{-1}(-2 i m \tilde{\Omega})^{-h} \Gamma\left(h-\frac{i \omega}{\kappa}\right)\left(-\tan \frac{\zeta}{2}\right)^{\frac{i \omega}{\kappa}} e^{i m \tilde{\Omega} \cot \zeta} W_{\frac{i \omega}{\kappa}, h-\frac{1}{2}}(2 i m \tilde{\Omega} \csc \zeta) .
\end{aligned}
$$

In the second line, we performed the change of variables $\bar{t}=\frac{1}{\kappa} \log \left(-\frac{(1+x) \tan \frac{\zeta}{2}}{x}\right)$, which allowed to recognize the integral representation of the hypergeometric $U$ function. We then used the definition of the Whittaker $W$ function, $W_{a, b}(z)=e^{-\frac{z}{2}} z^{b+\frac{1}{2}} U\left(b-a+\frac{1}{2}, 1+2 b, z\right)$. By using the QNM approximation (4.43), we find the waveform to be

$$
\begin{aligned}
\delta \psi_{4}(\hat{r} \rightarrow \infty)= & \sum_{l m}(-i m \tilde{\Omega})^{h} M^{2} \lambda^{h} k_{1} \frac{\mathcal{Z}}{R_{0}^{4} W} S_{l m}(\theta) e^{i m \hat{\phi}} e^{-i \frac{m-i \lambda h}{2 M} \hat{u}} e^{-i m \tilde{\Omega} \tan \frac{\zeta}{2}} \hat{x}^{-1} \\
& \times\left(\tan \frac{\zeta}{2} \csc \zeta\right)^{h}\left(\frac{1+\tan \frac{\zeta}{2} e^{-\lambda \hat{u} /(2 M)}}{2}\right)^{-2 h} e^{2 i m \tilde{\Omega} \csc \zeta \frac{\tan \frac{\zeta}{2} e^{-\lambda \hat{u} /(2 M)}}{1+\tan \frac{\zeta}{2} e^{-\lambda \hat{u} /(2 M)}}}
\end{aligned}
$$

after resumming the QNM using (D.20). The dictionary with physical orbit parameters is given in B.32).

In particular, in the case where $\zeta=-\frac{\pi}{2}$, we have more simply

$$
\begin{aligned}
B & =\frac{1}{\sqrt{2 \pi}}\left(\frac{\kappa}{2}\right)^{h}(-2 i m \Omega)^{h} \frac{\mathcal{Z}}{R_{0}^{4} W} \int_{0}^{\infty} \mathrm{d} \bar{t} e^{i \omega \bar{t}}\left(\sinh \frac{\kappa \bar{t}}{2}\right)^{-2 h} e^{i m \Omega \operatorname{coth} \frac{\kappa \bar{t}}{2}} \\
& =\frac{2^{h}}{\sqrt{2 \pi}} \kappa^{h-1} \frac{\mathcal{Z}}{R_{0}^{4} W} \Gamma\left(h-\frac{i \omega}{\kappa}\right) W_{\frac{i \omega}{\kappa},-h+\frac{1}{2}}(-2 i m \tilde{\Omega})
\end{aligned}
$$

after using (D.3) in Appendix $\mathrm{D}$ with $p=-i \omega / \kappa, z=\kappa \bar{t}$ and $y=-i m \tilde{\Omega}$. We can then compute the overtone sum explicitly using (D.20) to obtain

$$
\delta \psi_{4}(\hat{r} \rightarrow \infty)=\sum_{l m}(-i m \tilde{\Omega})^{h} k_{1} \frac{\lambda^{h} M^{2} \mathcal{Z}}{R_{0}^{4} W} S_{l m}(\theta) e^{i m \hat{\phi}} \hat{x}^{-1}\left(\frac{1-e^{-\frac{\lambda \hat{u}}{2 M}}}{2}\right)^{-2 h} e^{-i \frac{m-i \lambda h}{2 M} \hat{u}} e^{i m \tilde{\Omega} \operatorname{coth} \frac{\lambda \hat{u}}{4 M}}
$$

consistently with 6.61 when $\zeta=-\frac{\pi}{2}$. The dominant late time behavior is now

$$
\left|\delta \psi_{4}(\hat{r} \rightarrow \infty)\right| \propto \frac{\lambda^{1 / 2}}{\sinh \frac{\lambda \hat{u}}{4 M}}
$$




\subsection{Circular near-NHEK orbit to near-NHEK orbits}

There is only one set of orbits to consider.

Osculating $(e, \ell) /$ Plunging $(e, \ell)$ The transformation (B.36) which maps a Plunging $(e, \ell)$ orbit to a near-NHEK circular orbit behaves asymptotically as

$$
\begin{aligned}
r & =\bar{r}(\sinh \kappa \bar{t}+\chi(\cosh \kappa \bar{t}-1))\left(1+O\left(\bar{r}^{-1}\right)\right) \\
t & =-\frac{1}{\kappa} \log \left(\operatorname{coth} \frac{\kappa \bar{t}}{2}+\chi\right)\left(1+O\left(\bar{r}^{-1}\right)\right), \\
\phi & =\bar{\phi}\left(1+O\left(\bar{r}^{-1}\right)\right)
\end{aligned}
$$

where $\chi$ is related to the orbit parameters as (B.37). To match to the asymptotically flat space in the Kinnersley tetrad, this transformation needs to be accompanied by type III rotation characterized by

$$
F=\frac{\sqrt{\bar{r}(\bar{r}+2 \kappa)}}{-\chi \sqrt{\bar{r}(\bar{r}+2 \kappa)}+(\kappa+(\kappa+\bar{r}) \chi) \cosh \kappa \bar{t}+(\kappa+\bar{r}+\kappa \chi) \sinh \kappa \bar{t}} .
$$

Together with the near-NHEK circular solution (5.12) and (4.33) this leads to

$$
B=\frac{1}{\sqrt{2 \pi}} \frac{\tilde{\mathcal{Z}}}{\left(r_{0}\left(r_{0}+2 \kappa\right)\right)^{2} \tilde{W}} \int_{0}^{\infty} \mathrm{d} \bar{t} e^{i \omega \bar{t}}\left(\operatorname{coth} \frac{(\kappa \bar{t})}{2}+\chi\right)^{\frac{i m \tilde{\omega}}{k}}(\sinh (\kappa \bar{t})+\chi(\cosh (\kappa \bar{t})-1))^{-h} .
$$

For $\chi>-1$ it becomes, using (D.5) from Appendix $\mathrm{D}$ with $p=-i \omega / \kappa, z=\kappa \bar{t}$ and $y=-i m \tilde{\omega} / \kappa$,

$$
\begin{aligned}
B & =\frac{1}{\sqrt{2 \pi}} \frac{\tilde{\mathcal{Z}}}{\left(r_{0}\left(r_{0}+2 \kappa\right)\right)^{2} \tilde{W}} \kappa^{-1}(1+\chi)^{\frac{i m \tilde{\omega}}{\kappa}-h} 2^{h} \\
& \times B\left(h-\frac{i \omega}{\kappa}, 1-h-\frac{i m \tilde{\omega}}{\kappa}\right)_{2} F_{1}\left(h-\frac{i m \tilde{\omega}}{\kappa}, h-\frac{i \omega}{\kappa}, 1-\frac{i m \tilde{\omega}}{\kappa}-\frac{i \omega}{\kappa},-\frac{1-\chi}{1+\chi}\right)
\end{aligned}
$$

where $B(x, y)=\Gamma(x) \Gamma(y) / \Gamma(x+y)$ is the beta function.

In the QNM mode approximation, the overtone sum

$$
\begin{aligned}
& \sum_{N=0}^{\infty} \frac{(-1)^{N} e^{-i \frac{m-i \lambda(N+h)}{2 M} \hat{u}}}{N !} \frac{\Gamma\left(1-h-\frac{i m \tilde{\omega}}{\kappa}\right)}{\Gamma\left(1-h-\frac{i m \tilde{\omega}}{\kappa}-N\right)}{ }_{2} F_{1}\left(h-\frac{i m \tilde{\omega}}{\kappa},-N, 1-\frac{i m \tilde{\omega}}{\kappa}-N-h,-\frac{1-\chi}{1+\chi}\right) \\
& =e^{-i \frac{m-i \lambda h}{2 M} \hat{u}}\left(1+\frac{1-\chi}{1+\chi} e^{-\frac{\lambda \hat{u}}{2 M}}\right)^{-h+\frac{i m \tilde{\omega}}{\kappa}}\left(1-e^{-\frac{\lambda \hat{u}}{2 M}}\right)^{-h-\frac{i m \tilde{\omega}}{\kappa}}
\end{aligned}
$$


is computed by (D.16) with

$$
c_{+}=h+\frac{i m \tilde{\omega}}{\kappa}, \quad c_{-}=h-\frac{i m \tilde{\omega}}{\kappa}, \quad z=-\frac{1-\chi}{1+\chi}, \quad x=e^{-\frac{\lambda \hat{u}}{2 M}} .
$$

resulting in

$$
\begin{aligned}
\delta \psi_{4}(\hat{r} \rightarrow \infty)= & \sum_{l m} M^{2} 2^{h} \lambda^{h} \kappa^{-h} k_{1} \frac{\tilde{\mathcal{Z}}}{\left(r_{0}\left(r_{0}+2 \kappa\right)\right)^{2} \tilde{W}} S_{l m}(\theta) e^{i m \hat{\phi}} \hat{x}^{-1} e^{-i \frac{m-i \lambda h}{2 M} \hat{u}} \\
& \times\left(1+\chi+(1-\chi) e^{\left.-\frac{\lambda \hat{u}}{2 M}\right)^{-h+\frac{i m \tilde{\omega}}{\kappa}}}\left(1-e^{\left.-\frac{\lambda \hat{u}}{2 M}\right)^{-h-\frac{i m \tilde{\omega}}{\kappa}}} .\right.\right.
\end{aligned}
$$

The metric perturbation can also be obtained easily in the limit $\lambda \rightarrow 0$ by double integration over $\hat{u}$. Indeed, the QNM frequency $\hat{\omega} \approx \frac{m}{2 M}$ so from (C.18) and because the evolution timescale becomes exceedingly long with respect to the oscillation timescale we can just multiply the expression for each mode $m$ by $-2(2 M)^{2} / m^{2}$ to obtain

$$
\begin{aligned}
\left.\left(h_{+}-i h_{\times}\right)\right|_{\hat{r} \rightarrow \infty}= & -\sum_{l m} \frac{2^{h+3}}{m^{2} \kappa^{h}} k_{1} \frac{M^{4} \lambda^{h} \tilde{\mathcal{Z}}}{\left(r_{0}\left(r_{0}+2 \kappa\right)\right)^{2} \tilde{W}} S_{l m}(\theta) e^{i m \hat{\phi}} \hat{x}^{-1} e^{-i \frac{m-i \lambda h}{2 M} \hat{u}} \\
& \times\left(1+\chi+(1-\chi) e^{\left.-\frac{\lambda \hat{u}}{2 M}\right)^{-h+\frac{i m \tilde{\omega}}{\kappa}}}\left(1-e^{-\frac{\lambda \hat{u}}{2 M}}\right)^{-h-\frac{i m \tilde{\omega}}{\kappa}}\right.
\end{aligned}
$$

where we fixed the two integration constants (proportional to $\hat{u}^{0}$ and $\left.\hat{u}^{1}\right)$ so that $\left(h_{+}-\right.$ $\left.i h_{\times}\right)\left.\right|_{\hat{r} \rightarrow \infty} \rightarrow 0$ at $\hat{u} \rightarrow \infty$.

The dominant late time behavior is now

$$
\left|\delta \psi_{4}(\hat{r} \rightarrow \infty)\right| \propto \frac{\lambda^{1 / 2}}{\left(\sinh \frac{\lambda \hat{u}}{4 M}\right)^{1 / 2}\left(\cosh \frac{\lambda \hat{u}}{4 M}+\chi \sinh \frac{\lambda \hat{u}}{4 M}\right)^{1 / 2}} .
$$

The perturbation is better expressed in terms of the physical quantities: $\hat{E}$, the energy of the plunging body in the asymptotic frame and $\ell$ its angular momentum. Using (B.37) and $(3.1)$, we get

$$
\chi=\frac{2(2 M \hat{E}-\ell)}{\lambda \sqrt{3\left(\ell^{2}-\ell_{*}^{2}\right)}}, \quad \frac{1}{\kappa_{0}} \equiv \frac{r_{0}}{\kappa}=\frac{2 \ell}{\sqrt{3\left(\ell^{2}-\ell_{*}^{2}\right)}}-1 .
$$

When expressed as a function of $\kappa_{0}$, the perturbation (6.74) is independent of $r_{0}$, which cancels out.

\subsection{Summary and comparison}

In this section we have computed the coefficient $B$ that enters in the expressions $(4.23)$ and (4.35) for the asymptotic curvature perturbation sourced by a massive probe object on a 


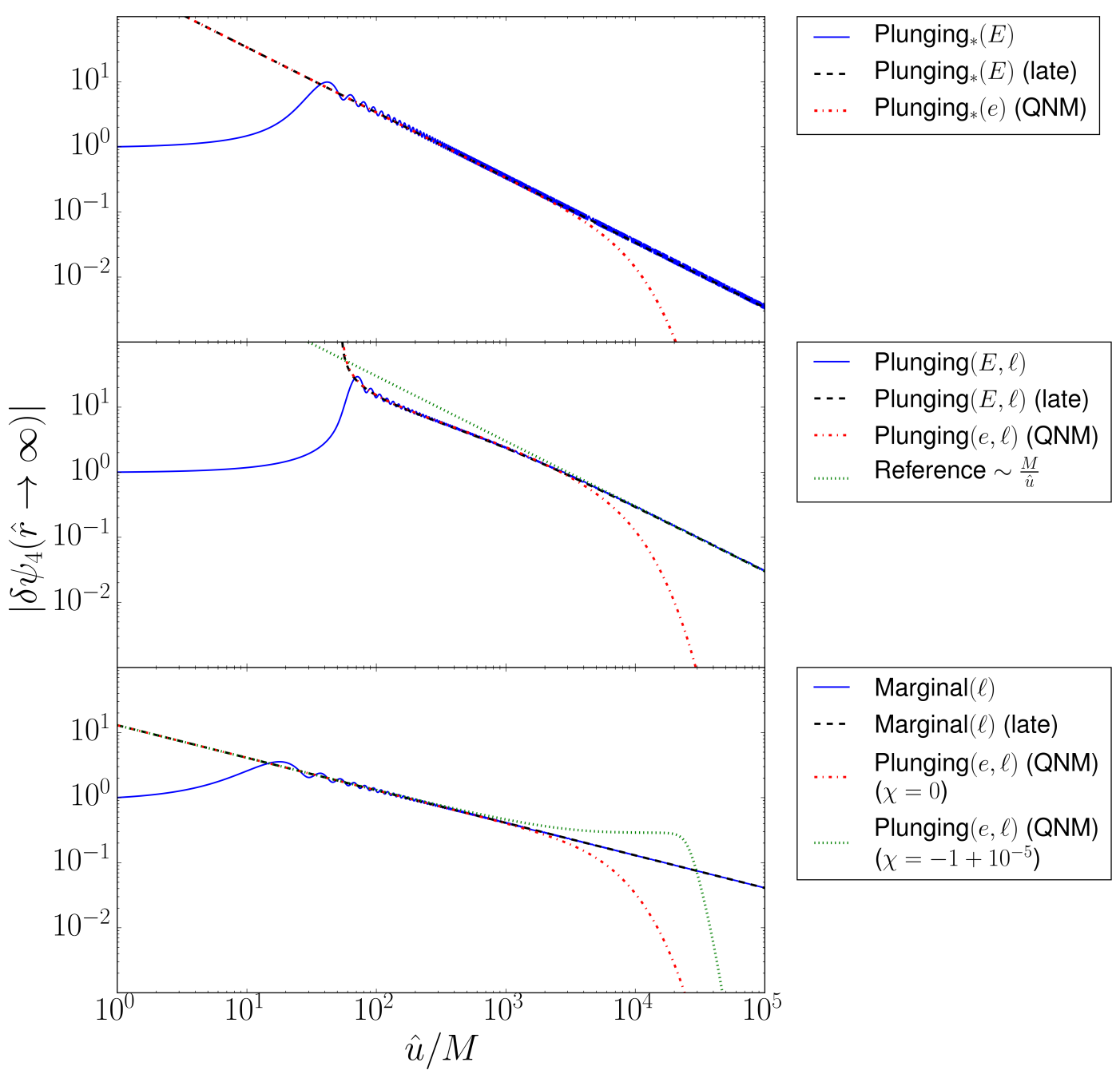

Figure 7: Envelope time evolution of the $l=2, m=2$ asymptotic curvature perturbation $\left|\delta \psi_{4}(\hat{r} \rightarrow \infty)\right|$ (artificially normalized to $\left.\left|\delta \psi_{4}(\hat{r} \rightarrow \infty)\right|_{\mid \hat{u}=M}=1\right)$, sourced by probe objects moving on a number of different geodesics in the near-horizon region of a nearly extremal black hole. We have taken $\lambda=10^{-3}$ in all panels. The solid (blue) lines show the time evolution corresponding to (from top to bottom) the NHEK orbits (6.47) $\left(E=2 \ell_{*}\right.$ ), (6.40) $\left(E=\frac{3 \ell_{*}}{4}, \ell=2 \ell_{*}\right)$ and 6.26) $\left(\ell=2 \ell_{*}\right)$. The dashed (black) lines are late time approximations to these expressions. The dashed-dotted (red) lines represent the curvature perturbations due to analogous near-NHEK orbits in the QNM approximations ( 6.63 and (6.73)). The dotted (green) line finally indicates the $\hat{u}^{-1}$ behavior (central panel) to which the signal from generic Plunging $(E, \ell)$ orbits asymptotes, and (bottom panel) the $(Q N M)$ response to a different Plunging $(E, \ell)$ orbit with a $\hat{u}^{-1 / 2}$ fall-off. The near-NHEK orbits transition from polynomial to exponential decay at times of order $\hat{u} \sim M \lambda^{-1}$. 
generic corotating equatorial orbit in NHEK or near-NHEK. This solves the problem of gravitational wave emission from such sources to leading order in the extremality asymptotic matched expansion scheme, and in the frequency domain, for which the matching condition (4.5) holds. From this we also have computed the corresponding GW signal in the time-domain, for sufficiently late times, for sources in both NHEK and near-NHEK.

In Figure 7 we combine the different features which together govern the qualitative time evolution of individual modes of the gravitational perturbation $|\delta \psi(\hat{r} \rightarrow \infty)|$ in the asymptotically flat domain, while ignoring the overall normalization constant which will be discussed in Section 7. Shown in Figure 7 is the normalized time evolution of the $l=2, m=2$ mode for a number of representative examples of orbits in NHEK and nearNHEK. One sees that once the peak amplitude is reached, the overall evolution is almost immediately captured by the late times approximate waveforms we discussed, except for an additional amplitude modulation, which we could not integrate analytically.

One typical signature is the polynomial decay of the signal. The signals from the Marginal $(\ell)$ and Plunging $(E)$ NHEK orbits decay respectively as $\hat{u}^{-1 / 2}$ and $\hat{u}^{-1}$. The signal from more general Plunging $(E, \ell)$ orbits generically interpolates between an initial $\hat{u}^{-1 / 2}$ behavior and a final $\hat{u}^{-1}$ decay, where the $\hat{u}^{-1 / 2}$ behavior becomes more and more pronounced as $E \rightarrow 0$. The polynomial ringdown stages of the near-NHEK counterparts to these NHEK signal are, at least on this level of single mode envelope evolution, completely analogous. For $e \rightarrow 0$, the Plunging $(e, \ell)$ signal decays like $\hat{u}^{-1 / 2}$ for the entire range $-\frac{\kappa}{2} \sqrt{3\left(\ell^{2}-\ell_{*}^{2}\right)}<e<0$ while the Plunging ${ }_{*}(e)$ orbit behaves as $\hat{u}^{-1}$. More general Plunging $(e, \ell)$ orbits give rise to polynomial ringdowns which exactly match the functional time dependence of the Plunging $(E, \ell)$ NHEK orbits, going from $\hat{u}^{-1 / 2}$ to $\hat{u}^{-1}$. The matching of parameters between both cases is qualitatively given by $\lambda^{1 / 3} \chi \sim \sin \zeta$.

Figure 7 shows that the near-NHEK signals transition from polynomial to exponential decay at times of order $\hat{u} \sim M \lambda^{-1}$. This corresponds to the timescale of the lowest zero-damped QNM given in 4.38). At this point, the NHEK and near-NHEK results diverge from each other. This is precisely what one expects because on such timescales, the spacings between the zero-damped QNMs are resolved. Therefore, the corresponding frequency differences make an important contribution to the signal. Since the NHEK limit ignores these differences the NHEK waveforms become unreliable at such late times.

In the next section we turn to the overall amplitudes of the GW signals in the asymptotically flat domain.

\section{Critical behavior}

The critical angular momentum per unit probe mass $\ell_{*}=\frac{2}{\sqrt{3}} M$ has taken on a special role throughout our computations. It is time to explicitly show that critical behavior occurs at and around $\ell=\ell_{*}$. We have seen that exactly two representative orbits under (complexified) $S L(2, \mathbb{R}) \times U(1)$ conformal transformations control the physics of probe 
orbits in the near-horizon region of nearly extremal Kerr: the circular orbit in NHEK which is critical, with $\ell=\ell_{*}$, and the family of circular orbits in near-NHEK which are supercritical, $\ell>\ell_{*}$.

The overall amplitude of the supercritical plunging orbits discussed in Sections 6.1 and 6.4 depend on the following coefficients

$$
\mathcal{A}_{l m}^{\ell>\ell_{*}}=\frac{8 M^{5} k_{1} \tilde{\mathcal{Z}}}{m^{2} \kappa^{h} m_{0} r_{0}^{2}\left(r_{0}+2 \kappa\right)^{2} \tilde{W}} .
$$

However one can verify that $M, m_{0}$ and $r_{0}$ (but not $\kappa / r_{0}$ ) explicitly drop out in this expression, leaving us with coefficients that depend on the physical impact parameters of the orbit only; the energy, angular momentum and initial plunging time.

Similarly, the overall amplitude of the critical plunging orbits discussed in Sections 6.2 and 6.3 depend upon the overall coefficients

$$
\mathcal{A}_{l m}^{\ell=\ell_{*}}=\frac{8 M^{5} k_{1} \mathcal{Z}}{m^{2} m_{0} R_{0}^{4} W}\left(-2 i m \tilde{\Omega} / R_{0}\right)^{h}
$$

which likewise involve the physical impact parameters of the orbit only ${ }^{16}$. We now derive the critical behavior associated with the plunging orbits on a case by case basis, by explicitly evaluating these coefficients.

The gravitational wave signal emitted by plunging probes strongly depends upon the $\theta$ incidence angle. We consider the two borderline cases: face-on $(\theta=0)$ and edge-on $\left(\theta=\frac{\pi}{2}\right)$. We take for simplicity $\hat{\phi}=0$.

In the face-on case, the leading signal comes from the $m=l=2$ harmoni ${ }^{17}$. The generic plunging supercritical near-NHEK orbits denoted as Plunging $(e, \ell)$ leads to the metric perturbation (6.74) consisting of a polynomial ringdown followed by an exponential decay. All in all, the dominant behavior for a generic orbit is given by

$$
\left|h_{+}+i h_{\times}\right|=\mathcal{A}_{F O}\left(\frac{\ell}{\ell_{*}}\right) \frac{m_{0}}{D} \frac{\sqrt{\lambda}}{\left(\sinh \frac{\lambda \hat{u}}{4 M}\right)^{1 / 2}\left(\cosh \frac{\lambda \hat{u}}{4 M}+\chi \sinh \frac{\lambda \hat{u}}{4 M}\right)^{1 / 2}}
$$

where $D=M \hat{x}=r$ is the luminosity distance to the source, $m_{0}$ is the mass of the probe and $\mathcal{A}_{F O}\left(\frac{\ell}{\ell_{*}}\right)=2^{-1 / 2}\left|\mathcal{A}_{22}^{\ell>\ell_{*}}\right| S_{22}(0)$ is the residual numerical coefficient which only depends upon the ratio $\ell / \ell_{*}$.

We obtain numerically for close to critical and large orbital angular momentum,

$$
\begin{aligned}
\mathcal{A}_{F O}\left(\frac{\ell}{\ell_{*}} \rightarrow 1\right) & =1.1\left(\frac{\ell}{\ell_{*}}-1\right)^{-\frac{1}{4}}, \\
\mathcal{A}_{F O}\left(\frac{\ell}{\ell_{*}} \rightarrow \infty\right) & =0.2 \frac{\ell}{\ell_{*}} .
\end{aligned}
$$

\footnotetext{
${ }^{16}$ We remind the reader that in all Figures in the previous section, these overall coefficients were artificially suppressed by a unit normalization of the amplitude.

${ }^{17}$ This is because the higher ( $\left.\operatorname{spin} 2\right)$ spheroidal harmonics $S_{l m}(0)$ are highly suppressed: the next-toleading terms are $S_{33}(0) \simeq 10^{-10}, S_{44}(0) \simeq 10^{-20}, \ldots$
} 


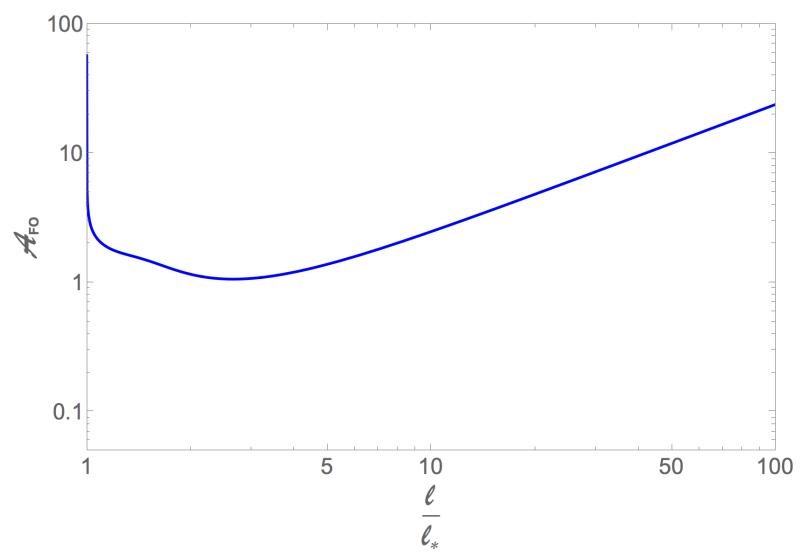

Figure 8: Amplitude coefficient (face-on) exhibits critical behavior in the limit $\ell \rightarrow \ell_{*}$

The minimum value of the amplitude occurs around $\ell=2.6 \ell_{*}$ where $\mathcal{A}_{F O}=1.1$. The function $\mathcal{A}_{F O}\left(\frac{\ell}{\ell_{*}}\right)$ is plotted in Figure 8. The behavior in the limit $\ell \rightarrow \ell_{*}$ is the critical behavior that we anticipated above, with critical exponent $-1 / 4$.

If the near-NHEK energy $e$ is finite and non-zero around the critical point $\ell \rightarrow \ell_{*}, \chi$ in (7.3) diverges (see (B.37)). The second term in the denominator in $(7.3)$ then dominates and diverges, and exactly cancels the divergence in $\mathcal{A}_{F O}$. This yields

$$
\lim _{\ell \rightarrow \ell_{*}, e \text { finite }}\left|h_{+}+i h_{\times}\right|=1.2 \frac{m_{0}}{D} \sqrt{\frac{\kappa \ell_{*}}{e}} \frac{\sqrt{\lambda}}{\sinh \frac{\lambda \hat{u}}{4 M}} .
$$

Again, we find critical behavior in the limit $\ell \rightarrow \ell_{*}$ and $e \rightarrow 0$. For the special case $\hat{E}=\frac{\ell}{2 M}$, or equivalently $\chi=0$, the near-NHEK energy $e=0$ and 7.3 gives the critical behavior

$$
\lim _{\ell \rightarrow \ell_{*}, e=0}\left|h_{+}+i h_{\times}\right|=1.6\left(\frac{\ell}{\ell_{*}}-1\right)^{-\frac{1}{4}} \frac{m_{0}}{D} \frac{\sqrt{\lambda}}{\sqrt{\sinh \frac{\lambda \hat{u}}{2 M}}} .
$$

As expected from a critical system, the asymptotic behavior close to the critical point depends on how the critical point is approached.

In the NHEK region, the waveform of the Plunging $(E, \ell)$ orbits has the dominant behavior

$$
\left|h_{+}+i h_{\times}\right|=\mathcal{A}_{F O}^{N H E K}\left(\frac{\ell}{\ell_{*}}\right) \frac{m_{0}}{D} \frac{\lambda^{\frac{1}{3}}}{\sqrt{\left|\left(\frac{\hat{u} \lambda^{2 / 3}}{4 M} \cos \frac{\zeta}{2}-\frac{1}{2} \sin \frac{\zeta}{2}\right)\left(\cos \frac{\zeta}{2}+\sin \frac{\zeta}{2} \frac{\hat{u} \lambda^{2 / 3}}{2 M}\right)\right|}}
$$

where $\mathcal{A}_{F O}^{N H E K}\left(\frac{\ell}{\ell}\right)=2^{-1 / 2}\left|\mathcal{A}_{22}^{\ell>\ell_{*}}\right| S_{22}(0)$. The overall amplitude is numerically equal to (7.4) and (7.5). We can relate $\zeta$ to impact parameters using (B.35). The amplitude depends upon the NHEK energy $E$ and the initial value $\bar{T}_{0}$. 
Now, for $\ell=\ell_{*}$, the plunging orbits belong to a different class: the Plunging $(e)$ or Plunging $_{*}(e=0)$ orbits, whose amplitude is now controlled by the other coefficient $(7.2)$. The face-on amplitude of perturbations is easily obtained from (6.63) and (6.57) and given by

$$
\begin{aligned}
\left.\left|h_{+}+i h_{\times}\right|\right|_{\ell=\ell_{*}, e \neq 0} & =\hat{\mathcal{A}}_{F O} \sqrt{\frac{\kappa \ell_{*}}{e}} \frac{m_{0}}{D} \frac{\sqrt{\lambda}}{\sinh \frac{\lambda \hat{u}}{4 M}}, \\
\left.\left|h_{+}+i h_{\times}\right|\right|_{\ell=\ell_{*}, e=0} & =\hat{\mathcal{A}}_{F O} \sqrt{\frac{e^{\kappa \bar{t}_{0}}}{\kappa}} \frac{m_{0}}{D} \sqrt{2 \lambda} e^{-\frac{\lambda \hat{u}}{4 M}}
\end{aligned}
$$

where we used the map of parameters (B.30)-(B.32) (with final barred coordinates) and defined $\hat{\mathcal{A}}_{F O}=2^{-1 / 2}\left|\mathcal{A}_{22}^{\ell=\ell_{*}}\right| S_{22}(0)$. A special feature of the amplitude of the Plunging $(e=$ $0)$ orbit is that it depends on the initial value $\bar{t}_{0}$ of the time parameter defined in (B.8). We obtain numerically $\hat{\mathcal{A}}_{F O}=1.2$.

As a last example of face-on amplitude, the Plunging ${ }_{*}(E)$ orbits leads to the overall signa 18

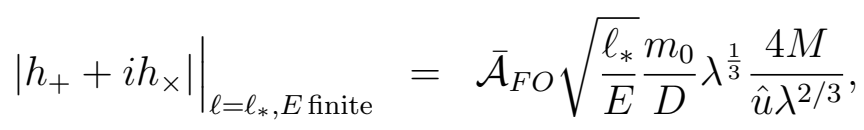

where $\overline{\mathcal{A}}_{F O}=2^{-1 / 2}\left|\mathcal{A}_{22}^{\ell=\ell_{*}}\right| S_{22}(0)$.

In the edge-on case, the leading contribution to the signal comes from a large number of harmonics which admit conformal weights with real part $1 / 2^{19}$. For the generic Plunging $(e, \ell)$ orbit, the dominant behavior of the envelope metric perturbation for a generic orbit is now given by

$$
\left|h_{+}+i h_{\times}\right|=\frac{m_{0}}{D} \frac{\sqrt{\lambda}}{\left(\sinh \frac{\lambda \hat{u}}{4 M}\right)^{1 / 2}\left(\cosh \frac{\lambda \hat{u}}{4 M}+\chi \sinh \frac{\lambda \hat{u}}{4 M}\right)^{1 / 2}}\left|\sum_{m=2}^{\infty} \mathcal{A}_{E O}^{m}\left(\frac{\ell}{\ell_{*}}, \hat{E} ; \frac{\hat{u}}{M} ; \lambda\right)\right|
$$

where

$$
\begin{aligned}
\mathcal{A}_{E O}^{m}\left(\frac{\ell}{\ell_{*}}, \hat{E} ; \frac{\hat{u}}{M} ; \lambda\right)= & -\sum_{l=m}^{l_{\max }(m)} 2^{-h} \lambda^{h-\frac{1}{2}} \mathcal{A}_{l m}^{\ell>\ell_{*}} S_{l m}\left(\frac{\pi}{2}\right) e^{-\frac{i m \hat{u}}{2 M}}\left(\sinh \frac{\lambda \hat{u}}{4 M}\right)^{\frac{1}{2}-h-\frac{i m \tilde{\omega}}{\kappa}} \\
& \times\left(\cosh \frac{\lambda \hat{u}}{4 M}+\chi \sinh \frac{\lambda \hat{u}}{4 M}\right)^{\frac{1}{2}-h+\frac{i m \tilde{\omega}}{\kappa}}
\end{aligned}
$$

In this expression, the dependence on $M, m_{0}$ and $r_{0}$ cancels out, but the dependence on

\footnotetext{
${ }^{18}$ Remember that $\hat{u} \lambda^{2 / 3}$ is kept fixed in the limit $\lambda \rightarrow 0, \hat{u} \rightarrow \infty$. Also, the overall scaling with $\lambda^{1 / 3}$ should be interpreted with care in the NHEK (but not near-NHEK) limit, see footnote 8

${ }^{19}$ The first such harmonics are $(l, m)=(2,2),(3,3),(4,4),(5,4),(5,5),(6,5),(6,6),(7,6),(7,7) \ldots$
} 

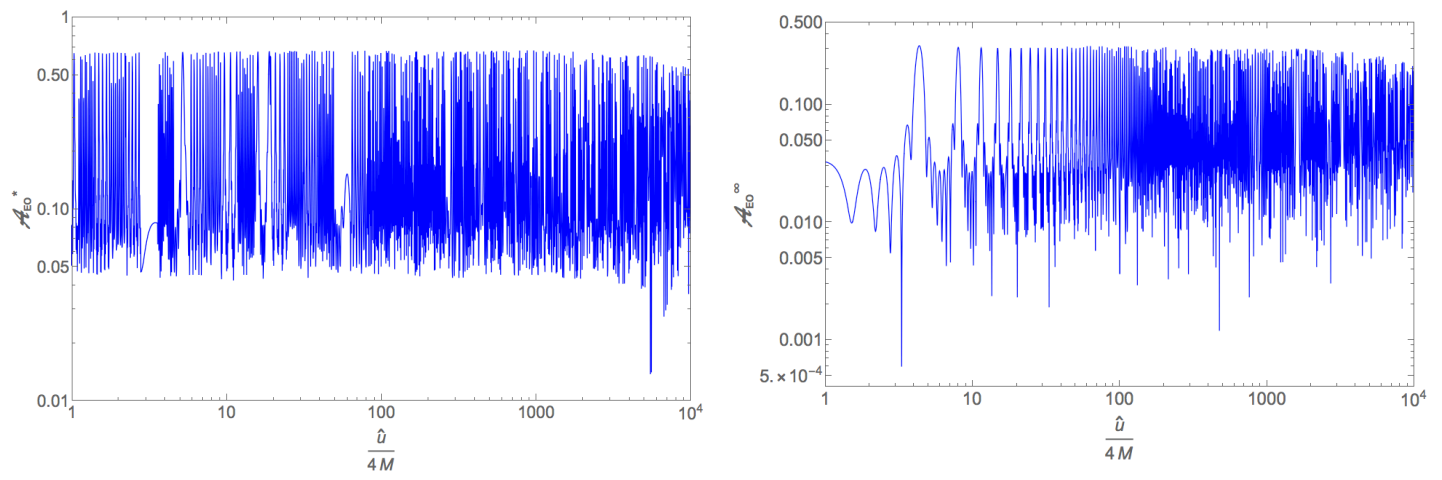

Figure 9: Amplitude coefficients (edge-on) for $\hat{E}=\frac{\ell_{*}}{2 M}$ and $\lambda=10^{-3}$. Left: near the critical limit $\ell \rightarrow \ell_{*}$. Right: for large specific angular momentum $\ell \rightarrow \infty$.

$\lambda$ remains. The upper bound $l_{\max }(m)$ in the sum in $(7.13)$ is the maximum value of $l$ such that $\operatorname{Re}(h)=1 / 2$. It is given by $\left\lfloor\frac{m}{0.754}\right\rfloor$ in the range $2 \leq m \leq 20$. Convergence of the amplitude requires a large $m$. We find numerically a convergence up to $\sim 5 \%$ by including all $2 \leq m \leq 20$. The overall amplitude in $(7.12)$ as a function of the ratio $\ell \rightarrow \ell_{*}$ exhibits similar (critical and linear) behavior as the face-on case,

$$
\begin{aligned}
\left|\sum_{m=2}^{\infty} \mathcal{A}_{E O}^{m}\left(\frac{\ell}{\ell_{*}} \rightarrow 1, \hat{E} ; \frac{\hat{u}}{M} ; \lambda\right)\right| & =\mathcal{A}_{E O}^{*}\left(\hat{E} ; \frac{\hat{u}}{M}\right)\left(\frac{\ell}{\ell_{*}}-1\right)^{-\frac{1}{4}}, \\
\left|\sum_{m=2}^{\infty} \mathcal{A}_{E O}^{m}\left(\frac{\ell}{\ell_{*}} \rightarrow \infty, \hat{E} ; \frac{\hat{u}}{M} ; \lambda\right)\right| & =\mathcal{A}_{E O}^{\infty}\left(\hat{E} ; \frac{\hat{u}}{M}\right) \frac{\ell}{\ell_{*}} .
\end{aligned}
$$

We plot $\mathcal{A}_{E O}^{*}\left(\hat{E} ; \frac{\hat{u}}{M}\right)$ and $\mathcal{A}_{E O}^{\infty}\left(\hat{E} ; \frac{\hat{u}}{M}\right)$ in Figure 9 for values $\hat{E}=\frac{\ell_{*}}{2 M}$ and $\lambda=10^{-3}$.

Similar critial behavior appears for generic Plunging $(E, \ell)$ orbits in NHEK region, we just write down the waveform

$$
\left|h_{+}+i h_{\times}\right|=\frac{m_{0}}{D} \frac{\lambda^{1 / 3}}{\sqrt{\left|\left(\frac{\hat{u} \lambda^{2 / 3}}{2 M} \cos \frac{\zeta}{2}-\sin \frac{\zeta}{2}\right)\left(\cos \frac{\zeta}{2}+\sin \frac{\zeta}{2} \frac{\hat{u} \lambda^{2 / 3}}{2 M}\right)\right|}} \times\left|\sum_{m=2}^{\infty} \mathcal{A}_{E O, N H E K}^{m}\left(\frac{\ell}{\ell_{*}}, \hat{E} ; \frac{\hat{u}}{M} ; \lambda\right)\right|,
$$

where

$$
\begin{aligned}
\mathcal{A}_{E O, N H E K}^{m}\left(\frac{\ell}{\ell_{*}}, \hat{E} ; \frac{\hat{u}}{M} ; \lambda\right)= & -\sum_{l=m}^{l_{\max }(m)} \mathcal{A}_{l m}^{\ell>\ell_{*}} S_{l m}\left(\frac{\pi}{2}\right) e^{-i \frac{m}{2 M} \hat{u}} \lambda^{\frac{2}{3}\left(h-\frac{1}{2}\right)} \\
& \times\left(\frac{\hat{u} \lambda^{2 / 3}}{2 M} \cos \frac{\zeta}{2}-\sin \frac{\zeta}{2}\right)^{\frac{1}{2}-h-i \frac{m \tilde{\omega}}{\kappa}}\left(\cos \frac{\zeta}{2}+\sin \frac{\zeta}{2} \frac{\hat{u} \lambda^{2 / 3}}{2 M}\right)^{\frac{1}{2}-h+i \frac{m \tilde{\omega}}{\kappa}} .
\end{aligned}
$$




\section{Observability by LISA}

Let us assume for a moment that nearly extremal supermassive black holes exist in Nature, preferably even at rather low redshift. It is then an interesting and timely question whether the gravitational wave signals from the final plunges of EMRIs that we have computed, are potentially observable by LISA. In this section we compute the signal to noise (SNR) ratio of our waveforms for parameters in the LISA band and assuming no prior knowledge about the orbit from the earlier inspiral. Of course this won't be the case in realistic situations, but it serves to estimate the observability of our waveforms as independent signals on their own. In the analysis of [11], the near-extremality parameter $\lambda=10^{-2}$ was assumed, since the near-horizon analytic result models up to $\sim 10 \%$ precision a full e-fold of the adiabatically evolved inspiral into Gargantua [11, 13]. In our analysis, given the lack of a parallel numerical analysis that would allow to check the precision of the near-horizon results for plunges, we will be more conservative and choose as a reference the near-extremality parameter $\lambda=10^{-3}$. The existence of such sources is more speculative, but our analytical results are more accurate.

The frequencies of the gravitational wave oscillations as seen from a detector in the asymptotically flat region will be harmonics of the angular frequency of the central, extremal Kerr black hole,

$$
f_{\infty}=\frac{\Omega_{e x t}}{2 \pi}=\frac{1}{4 \pi M}=1.6 \times 10^{-2} H z\left(\frac{10^{6} M_{\odot}}{M}\right) .
$$

with the lowest harmonics dominating the signal in the regime of interest. The typical LISA source masses $M$ lie in the range $10^{5}-10^{7} M_{\odot}$. In the corresponding range of frequencies $f_{\infty}$ the sky-averaged strain sensitivity of the LISA observatory expressed as power spectral density $S_{h}(f)$ is approximately constant and equal to $4 \times 10^{-40} \mathrm{~Hz}^{-1}[1]$.

The SNR of a monochromatic measured gravitational wave $h(t)$ is given by

$$
\left(\frac{S}{N}\right)^{2}=\frac{2}{S_{h}\left(\Omega_{e x t} / \pi\right)} \int_{\hat{u}_{i}}^{\hat{u}_{f}}|h(\hat{u})|^{2} d \hat{u}
$$

where $U \equiv \hat{u}_{f}-\hat{u}_{i}$ is the time over which the signal is measured.

We now use this to estimate the observability of the signal for a number of representative examples of waveforms associated with generic edge-on or face-on Plunging $(e, \ell)$ orbits for which both the polynomial and the onset of the exponential ringdown lie within the LISA range. Some of the waveforms are shown in Figure 10 and Figure 11.

We first consider the $e=0$, face-on plunging orbit with near-critical angular momentum $\ell \rightarrow \ell_{*}$ exhibiting critical behavior. Substituting its waveform (7.7) in (8.2) yields

$$
\left(\frac{S}{N}\right)^{2}=\frac{m_{0}^{2} M}{D^{2}}\left(\frac{\ell}{\ell_{*}}-1\right)^{-\frac{1}{2}} \frac{10.24}{S_{h}\left(\Omega_{e x t} / \pi\right)}\left(-\ln \left(\lambda \hat{u}_{i} / 4 M\right)\right)
$$



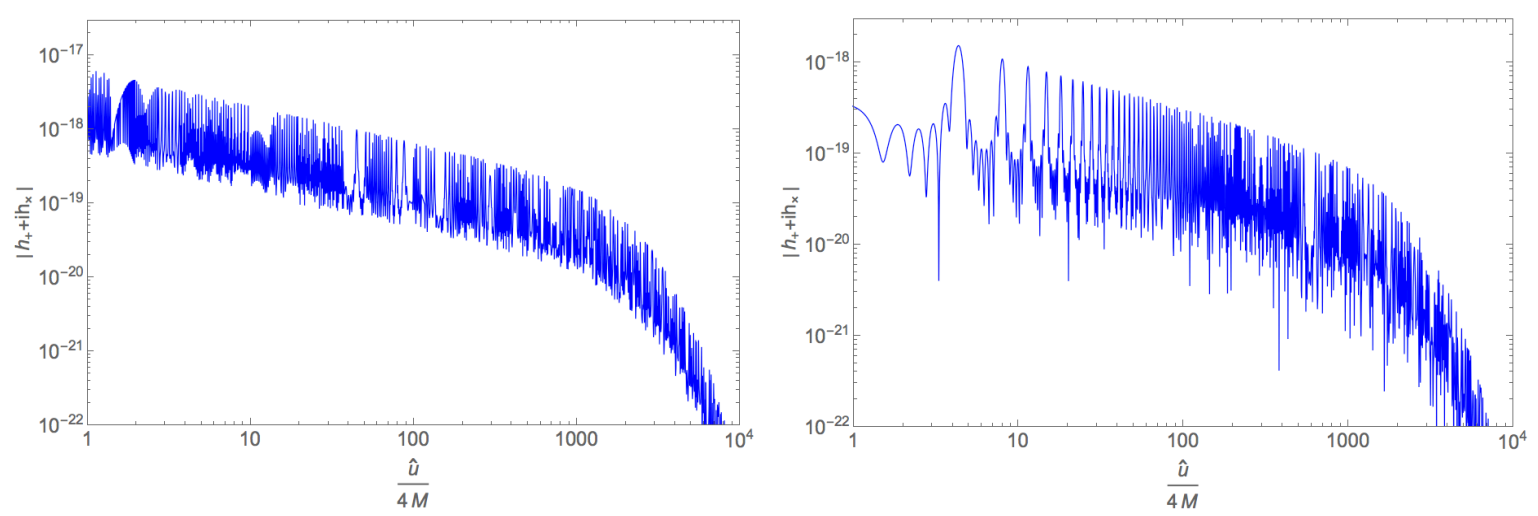

Figure 10: Strain for an edge-on Plunging $(e, \ell)$ orbit with $\hat{E}=\frac{\ell}{2 M}$ and $\lambda=10^{-3}$. Left: a nearly critical orbit with $\ell \rightarrow \ell_{*}$ and $\frac{m_{0}}{D}\left(\frac{\ell}{\ell_{*}}-1\right)^{-1 / 4}=10^{-17}$. Right: an orbit with large angular momentum $\ell$ and $\frac{m_{0}}{D} \frac{\ell}{\ell_{*}}=10^{-17}$.

where we have taken the final time $\hat{u}_{f}$ in $(8.2)$ well beyond the transition from polynomial to exponential decay, which occurs around $\hat{u} \sim 2 M / \lambda$. The initial time corresponds to the onset of the plunging near-NHEK regime. It could be determined by a numerical simulation. A conservative order of magnitude estimate is to take $\hat{u}_{i}$ to be a few times $M$. This timescale is certainly consistent with considering the zero-damped modes only, as the damped modes should typically decay on timescale of order $M$. To determine the LISA observable volume of this signal, consider a measurement at threshold SNR $\rho_{t h}$, which we assume to be around 15 . With $\lambda=10^{-3}$ and $\hat{u}_{i}=10 M$, this yields the maximal luminosity distance

$$
D_{F O, e=0}^{\max } \approx 0.08 \mathrm{Gpc}\left(\frac{M}{10^{7} M_{\odot}}\right)^{1 / 2}\left(\frac{m_{0}}{10 M_{\odot}}\right)\left(\frac{\ell}{\ell_{*}}-1\right)^{-\frac{1}{4}}\left(\frac{15}{\rho_{t h}}\right) .
$$

The result for face-on orbits in the large angular momentum $\ell \gg \ell_{*}$ takes a similar form but with the critical enhancement factor above replaced by the linear enhancement in (7.5).

Next consider an $e \neq 0$ face-on plunging orbit, again with near-critical angular momentum $\ell \rightarrow \ell_{*}$. Substituting the waveform (7.6) in 8.2 and integrating over the time over which the signal is measured yields the following SNR,

$$
\left(\frac{S}{N}\right)^{2}=\frac{m_{0}^{2} M}{\lambda D^{2}}\left(\frac{\kappa l_{*}}{e}\right)\left(\frac{M}{\hat{u}_{i}}\right) \frac{32}{S_{h}\left(\Omega_{e x t} / \pi\right)} .
$$

Taking again $\lambda=10^{-3}$ and $\hat{u}_{i}=10 M$, the distance to which we can expect LISA to 
detect the signal of a plunging orbit of this kind is

$$
D_{F O, e \neq 0}^{\max } \approx 0.63 \mathrm{Gpc}\left(\frac{M}{10^{7} M_{\odot}}\right)^{1 / 2}\left(\frac{m_{0}}{10 M_{\odot}}\right) \sqrt{\frac{\kappa \ell_{*}}{e}}\left(\frac{15}{\rho_{t h}}\right) .
$$

The observable volume $\sim\left(D^{\max }\right)^{3}$ of other face-on orbits can be obtained in a similar manner from the explicit formulae for the waveforms in Section 7 .

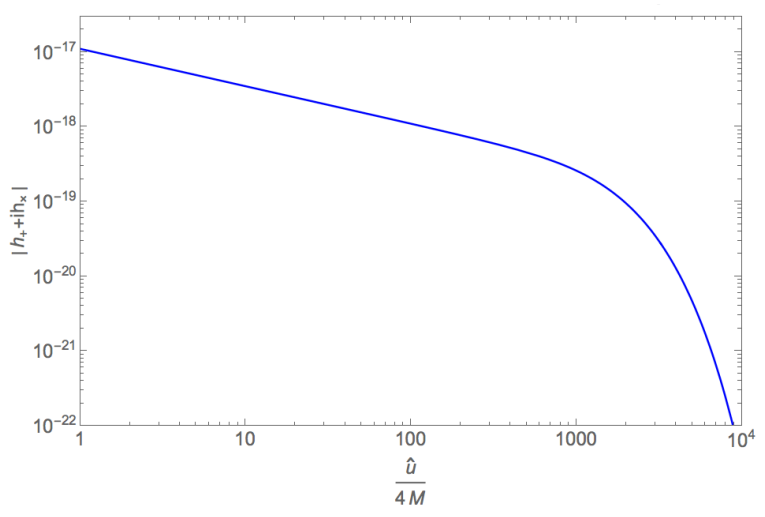

Figure 11: Strain for a face-on Plunging $(e, \ell)$ orbit with energy $\hat{E}=\frac{\ell}{2 M}, \lambda=10^{-3}$, $\ell=2.6 \ell_{*}$ and with $\frac{m_{0}}{D}=10^{-17}$.

We conclude with an example of a $\chi=0$ edge-on plunging orbit (cf. Figure 11), again with near-critical angular momentum $\ell \rightarrow \ell_{*}$. In this case a large number of modes significantly contribute to the measured signal. The waveform is given in (7.12) with amplitude (7.14) exhibiting critical behavior. Figure 10 shows that the amplitude factor $\mathcal{A}_{E O}^{*}$ rapidly oscillates over the time of observation around a constant value $\sim 0.1$. We note in passing that the amplitude of the signal is strongly $\theta$ dependent, which should allow for a precise sky localization of the signal. An estimate of the observability can be obtained by substituting the waveform in 8.2 , yielding

$$
\left(\frac{S}{N}\right)^{2}=\frac{m_{0}^{2} M}{D^{2}}\left(\frac{\ell}{\ell_{*}}-1\right)^{-\frac{1}{2}} \frac{8\left(\mathcal{A}_{E O}^{*}\right)^{2}}{S_{h}\left(\Omega_{\text {ext }} / \pi\right)}\left(-\ln \left(\lambda \hat{u}_{i} / 4 M\right)\right)
$$

which then gives, for $u_{i}=10 M$,

$$
D_{E O, e=0}^{\max } \approx 0.07 \operatorname{Gpc}\left(\frac{M}{10^{7} M_{\odot}}\right)^{1 / 2}\left(\frac{m_{0}}{10 M_{\odot}}\right)\left(\frac{\ell}{\ell_{*}}-1\right)^{-\frac{1}{4}}\left(\frac{15}{\rho_{t h}}\right) .
$$

We conclude that the maximal luminosity distance for edge-on or face-on signals is similar. The observable volume depends significantly on the enhancement factor, whose form is itself determined by the conformal class and the impact parameters of the orbit. Redshifts $z \sim 1$ or $D^{\max } \approx 7 \mathrm{Gpc}$ require a large enhancement factor associated with critical behavior. 


\section{Summary}

We have analytically computed the gravitational wave emission from the final stages of a class of EMRIs in which a non-spinning compact object on a generic corotating equatorial orbit enters the near-horizon (either NHEK or near-NHEK) region of Gargantua, i.e. a supermassive nearly extremal Kerr black hole.

To do so we have first enumerated nearly corotating test particle orbits in the equatorial plane of the near-horizon region of a nearly extremal Kerr black hole. We found three classes of plunging orbits in both NHEK and near-NHEK spacetimes, one class of circular orbits as well as one class of osculating orbits. We also found that there is a minimum angular momentum per unit probe mass $\ell_{*}=\frac{2}{\sqrt{3}} M$, which plays a fundamental role in our later analysis. This specific angular momentum coincides with the one of the ISCO and is therefore physically interesting. Studying these orbits in terms of complex representations of the conformal symmetry group $S L(2, \mathbb{R}) \times U(1)$, which leave the angular momentum invariant but change the energy, we found that there are only two conjugacy classes under complexified conformal symmetry combined with PT symmetry. Their representatives can be chosen to be the (stable) circular orbit in NHEK with critical angular momentum $\ell=\ell_{*}$ and the (unstable) circular orbit in near-NHEK with supercritical angular momentum $\ell>\ell_{*}$.

We have computed by brute force methods the exact curvature perturbation induced by a probe on a circular orbit in near-NHEK, thereby completing the twin computation of the circular orbit in NHEK [28]. Conformal symmetry allows one to obtain the gravitational waveforms for generic equatorial trajectories from the waveforms of these two circular "seed orbits" by employing complex transformations, as pioneered for a specific class of orbits and a real transformation in [29]. We have used these waveforms and the calculational power brought about by conformal symmetry to obtain analytic expressions for the leading order time domain gravitational waveforms from plunges on all corotating equatorial orbits into nearly extremal Kerr black holes. Conformal symmetry not only allows one to shorten the computation but also structurally implies that the waveforms involve analytically tractable integral representations of hypergeometric functions (or simpler) and tabulated inverse Laplace transforms.

The oscillation timescale of any gravitational perturbation originating from the nearhorizon region is set by the Lense-Thirring corotation scale of the central nearly extremal Kerr black hole, $\Omega_{\text {ext }}^{-1}=2 M$. This kinematical feature is a straightforward consequence of the near-horizon limit. A less obvious feature is that the asymptotic amplitude of the gravitational perturbation for all plunging orbits is suppressed as $\lambda^{\frac{2}{3}} \operatorname{Re}(h)$ in the particular NHEK scaling that preserves the ISCO, as does the emission from the ISCO orbit [11,28]. This suppression factor becomes universally $\lambda^{\operatorname{Re}(h)}$ in the near-NHEK limit. This means that the modes with conformal weight $h=\frac{1}{2}+i h_{I}, h_{I}<0$ provide the dominant contribution in the near-horizon region, leading to $\left|\delta \psi_{4}\right|=O\left(\lambda^{1 / 3}\right)$ and $O\left(\lambda^{1 / 2}\right)$, respectively.

A new "smoking gun" signature of the asymptotic GW signal from plunging orbits 
in the near-horizon region is the existence of two phases: a polynomial decay with a specific exponent, followed by an exponential decay. The polynomial phase arises from the coherent stacking of zero-damped quasi-normal modes [37]. We find that the polynomial decay generically interpolates between an initial $\hat{u}^{-1 / 2}$ behavior and a final $\hat{u}^{-1}$ fall-off, where $\hat{u}$ is the retarded asymptotic time. The details of this interpolation depend on the impact parameters. To the best of our knowledge this behavior has not been seen yet in numerical simulations (see however [47]). It should however be present sufficiently close to extremality, say for $\lambda \leq 10^{-3}$. In all near-NHEK orbits the polynomial decay is followed by a universal exponential decay with a characteristic timescale $\Delta \hat{u}=2 M / \lambda$ governed by the lowest zero-damped $\mathrm{QNM}^{20}$,

A second unique and remarkable feature concerns the amplitude of the asymptotic curvature perturbation sourced by plunges in the near-horizon region. It critically depends on $\ell$, the conserved angular momentum per unit rest mass of the orbit. In particular the amplitude of the GW signal grows linearly with $\ell$ when $\ell$ is large. By contrast it exhibits critical behavior when $\ell$ tends to its minimum value $\ell_{*}=\frac{2}{\sqrt{3}} M$, which corresponds to the physically relevant specific angular momentum of the ISCO orbit. This critical behavior comes with specific critical exponents, which we computed. The metric perturbations $h_{+}$and $h_{\times}$are approximately proportional to the curvature perturbation because the oscillation timescale $2 M$ is extremely short compared with the timescale of the overall evolution of the amplitude, $2 M / \lambda$. This means that the amplitude of the asymptotic GW signal inherits this critical behavior and in particular diverges in the limit $\ell \rightarrow \ell_{*}$. We intend to study the nature of this critical behavior in more detail in future work. It would be interesting for instance to study whether self-force effects regulate the gravitational wave emission and what would be the critical behavior including self-force effects. More work is also required to see a possible relationship of this near-extremal critical behavior with other critical phenomena in black hole mergers 4952 .

Our results for the gravitational waveforms generated in the final near-horizon stages of corotating equatorial EMRIs into Gargantua complete all previous results for scalar and gravitational probes $[11,13,28,31$. They exactly or qualitatively agree in all cases where comparison is possible. It would be interesting to explore numerically what is the range of values of the extremality parameter $\lambda$ for which our analytic waveforms are accurate. At any rate, our precise analytic results serve as benchmarks for effective EMRI waveform models and numerical simulations.

Whether observatories like LISA will observe GW signals from plunges into nearlyextremal Kerr black holes will depend not only on the range of $\lambda$ for which these signals are produced, but also on whether supermassive black holes in this range exist at all. The standard geometrically thin disk model does not produce massive black holes in this range in light of the Thorne bound [18]. However, other disk models might be realized in Nature. In particular systems sustained by magnetic fields can exceed the

\footnotetext{
${ }^{20}$ The NHEK limit does not resolve this exponential phase.
} 
bound [20,53]. Alternatively, high spin black holes are producable in black hole collisions [50, 54]. Assuming such black holes exist and assuming no prior knowledge about the orbit from the earlier inspiral, we find the LISA observable volume of these GW signals extends out to redshifts $z \sim 0.15$. Evidently this is significantly larger for nearly critical orbits or high orbital angular momentum orbits - if only we were so lucky...

Acknowledgments We thank Niels Warburton for making public his code for computing spheroidal harmonics and our referees for constructive suggestions. This work makes use of the Black Hole Perturbation Toolkit. We thank Sam Gralla, Achilleas Porfyriadis and Andy Strominger for their comments on the manuscript. This work was supported in part by the European Research Council grant ERC-2013-CoG 616732 HoloQosmos, the ERC Starting Grant 335146 "HoloBHC", by the C16/16/005 grant of the KU Leuven, and by the FWO grant G092617N. We also acknowledge networking support by the COST Action GWverse CA16104. G.C. is a Research Associate of the Fonds de la Recherche Scientifique F.R.S.-FNRS (Belgium).

\section{A Teukolsky's formalism}

Unless otherwise noted we use the conventions of [55]. In particular, we use the $(-+++)$ signature while most of the literature on Teukolsky's formalism uses $(+---)$ signature [56, 57. We also use Einstein's equations in the form $G_{\mu \nu}=+8 \pi T_{\mu \nu}$ which differ by a sign from [56]. Our convention for the normalization of spheroidal harmonics however differs from 55] and matches with 28].

\section{A.1 Newman-Penrose tetrad}

A Newman-Penrose tetrad consists of two real null vectors $l^{\mu}, n^{\mu}$, and one complex null vector $m^{\mu}$ satisfying $l^{\mu} n_{\mu}=-m^{\mu} \bar{m}_{\mu}=-1$ and such that $g_{\mu \nu}=-l_{\mu} n_{\mu}-n_{\mu} l_{\nu}+m_{\mu} \bar{m}_{\mu}+$ $\bar{m}_{\mu} m_{\nu}$. The directional derivatives along the tetrads are defined as ${ }^{21}$

$$
D=l^{\mu} \partial_{\mu}, \quad \Delta=n^{\mu} \partial_{\mu}, \quad \delta=m^{\mu} \partial_{\mu}, \quad \bar{\delta}=\bar{m}^{\mu} \partial_{\mu} .
$$

\footnotetext{
${ }^{21}$ We warn the reader that the notation $\Delta$ is also used for $(2.3), \delta$ is also used for denoting a variation and $\kappa$ and $\lambda$ are also defined in the (near)-NHEK limit. The meaning of each symbol should be clear in each context.
} 
The spin coefficients are defined as

$$
\begin{aligned}
& \kappa=-m^{\mu} l^{\nu} \nabla_{\nu} l_{\mu} \quad \sigma=-m^{\mu} m^{\nu} \nabla_{\nu} l_{\mu} \\
& \lambda=-n^{\mu} \bar{m}^{\nu} \nabla_{\nu} \bar{m}_{\mu} \quad \nu=-n^{\mu} n^{\nu} \nabla_{\nu} \bar{m}_{\mu} \\
& \rho=-m^{\mu} \bar{m}^{\nu} \nabla_{\nu} l_{\mu} \quad \mu=-n^{\mu} m^{\nu} \nabla_{\nu} \bar{m}_{\mu} \\
& \tau=-m^{\mu} n^{\nu} \nabla_{\nu} l_{\mu} \quad \varpi=-n^{\mu} l^{\nu} \nabla_{\nu} \bar{m}_{\mu} \\
& \epsilon=-\frac{1}{2}\left(n^{\mu} l^{\nu} \nabla_{\nu} l_{\mu}+m^{\mu} l^{\nu} \nabla_{\nu} \bar{m}_{\mu}\right) \\
& \gamma=-\frac{1}{2}\left(n^{\mu} n^{\nu} \nabla_{\nu} l_{\mu}+m^{\mu} n^{\nu} \nabla_{\nu} \bar{m}_{\mu}\right) \\
& \alpha=-\frac{1}{2}\left(n^{\mu} \bar{m}^{\nu} \nabla_{\nu} l_{\mu}+m^{\mu} \bar{m}^{\nu} \nabla_{\nu} \bar{m}_{\mu}\right) \\
& \beta=-\frac{1}{2}\left(n^{\mu} m^{\nu} \nabla_{\nu} l_{\mu}+m^{\mu} m^{\nu} \nabla_{\nu} \bar{m}_{\mu}\right)
\end{aligned}
$$

and the Weyl scalars are defined as

$$
\begin{aligned}
& \psi_{0}=C_{\alpha \beta \mu \nu} l^{\alpha} m^{\beta} l^{\mu} m^{\nu} \\
& \psi_{1}=C_{\alpha \beta \mu \nu} l^{\alpha} n^{\beta} l^{\mu} m^{\nu} \\
& \psi_{2}=C_{\alpha \beta \mu \nu} l^{\alpha} m^{\beta} \bar{m}^{\mu} n^{\nu} \\
& \psi_{3}=C_{\alpha \beta \mu \nu} l^{\alpha} n^{\beta} \bar{m}^{\mu} n^{\nu} \\
& \psi_{4}=C_{\alpha \beta \mu \nu} n^{\alpha} \bar{m}^{\beta} n^{\mu} \bar{m}^{\nu}
\end{aligned}
$$

For further use, remember that under a type III rotation of the spin frame

$$
l^{\mu} \rightarrow A^{-1} l^{\mu}, \quad n^{\mu} \rightarrow A n^{\mu}, \quad m^{\mu} \rightarrow e^{i \theta} m^{\mu}
$$

with $A, \theta$ arbitrary real numbers, the $\psi_{4}$ scalar transforms as [58]

$$
\psi_{4} \rightarrow A^{2} e^{-2 i \theta} \psi_{4} .
$$

For the Kerr black hole, we use Kinnersley's tetrad

$$
\begin{aligned}
\hat{l}^{\mu} & =\Delta^{-1}\left(\left(\hat{r}^{2}+a^{2}\right), \Delta, 0, a\right) \\
\hat{n}^{\mu} & =(2 \Sigma)^{-1}\left(\left(\hat{r}^{2}+a^{2}\right),-\Delta, 0, a\right) \\
\hat{m}^{\mu} & =(\sqrt{2}(\hat{r}+i a \cos \theta))^{-1}\left(i a \sin \theta, 0,1, \frac{i}{\sin \theta}\right)
\end{aligned}
$$

where we recall the definitions (2.3). With respect to this tetrad the spin coefficients are 
given by

$$
\begin{array}{rrr}
\kappa=\sigma=\lambda=\nu=\epsilon=0, & \beta=-\frac{\bar{\rho} \cot \theta}{2 \sqrt{2}}, \\
\rho=-\frac{1}{\hat{r}-i a \cos \theta}, & \mu=\frac{\rho \Delta}{2 \Sigma} \\
\tau=\frac{-i a \sin \theta}{\sqrt{2} \Sigma}, & \varpi=\frac{i a \rho^{2} \sin \theta}{\sqrt{2}}, \\
\alpha=\varpi-\bar{\beta}, & \gamma=\mu+\frac{(\hat{r}-M)}{2 \Sigma} .
\end{array}
$$

The Kerr solution is type D and out of the 5 Newman-Penrose scalars of the background only $\psi_{2}$ does not vanish for Kerr [59]. It is given by

$$
\psi_{2}=-\frac{M}{(\hat{r}-i a \cos \theta)^{3}} .
$$

In the Poincaré NHEK limit (2.7), we perform a tetrad rotation $\hat{l}^{\mu}=M \lambda^{2 / 3} L^{\mu}, \hat{n}^{\mu}=$ $\frac{1}{M \lambda^{2 / 3}} N^{\mu}, \hat{m}^{\mu}=M^{\mu}$. The resulting Kinnersley tetrad is given by

$$
\begin{aligned}
L^{\mu} & =\left(\frac{1}{R^{2}}, 1,0,-\frac{1}{R}\right), \\
N^{\mu} & =\frac{1}{2 M^{2}\left(1+\cos ^{2} \theta\right)}\left(1,-R^{2}, 0,-R\right) \\
M^{\mu} & =(\sqrt{2} M(1+i \cos \theta))^{-1}\left(0,0,1, \frac{i\left(1+\cos ^{2} \theta\right)}{2 \sin \theta}\right) .
\end{aligned}
$$

The spin coefficients read as

$$
\begin{array}{lrl}
\kappa=\sigma=\lambda=\nu=\epsilon=\mu=\rho=0, & \beta=\frac{\cot \theta}{2 \sqrt{2} M(1+i \cos \theta)}, \\
\tau=\frac{-i \sin \theta}{M \sqrt{2}\left(1+\cos ^{2} \theta\right)}, & \varpi=\frac{i \sin \theta}{M \sqrt{2}(1-i \cos \theta)^{2}}, \\
\alpha=\varpi-\bar{\beta}, & \gamma=\frac{R}{2 M^{2}\left(1+\cos ^{2} \theta\right)} .
\end{array}
$$

and all Newman-Penrose scalars are vanishing except

$$
\psi_{2}=-\frac{1}{M^{2}(1-i \cos \theta)^{3}}
$$

In the near-NHEK limit $(2.9)$, we perform a tetrad rotation $\hat{l}^{\mu}=\frac{M \lambda}{\kappa} l^{\mu}, \hat{n}^{\mu}=\frac{\kappa}{M \lambda} n^{\mu}$, 
$\hat{m}^{\mu}=m^{\mu}$. The Kinnersley tetrad is given by

$$
\begin{aligned}
l^{\mu} & =\left(\frac{1}{r(r+2 \kappa)}, 1,0,-\frac{(r+\kappa)}{r(r+2 \kappa)}\right), \\
n^{\mu} & =\frac{1}{2 M^{2}\left(1+\cos ^{2} \theta\right)}(1,-r(r+2 \kappa), 0,-(r+\kappa)), \\
m^{\mu} & =(\sqrt{2} M(1+i \cos \theta))^{-1}\left(0,0,1, \frac{i\left(1+\cos ^{2} \theta\right)}{2 \sin \theta}\right) .
\end{aligned}
$$

All spin coefficients are identical to A.23 except for

$$
\gamma=\frac{(r+\kappa)}{2 M^{2}\left(1+\cos ^{2} \theta\right)}
$$

We also have (A.24).

\section{A.2 Master equation of Teukolsky}

The Teukolsky master equation unifies the description of the linearized dynamics of various fields around a Kerr black hole or, more generally, a type D spacetime in a single partial differential equation. All gravitational perturbations are encoded in either $\delta \psi_{0}$ or $\delta \psi_{4}$ which are invariant under linearized diffeomorphisms. The only perturbations with $\delta \psi_{0}=\delta \psi_{4}=0$ are deformations which change $M$ or $J$, which introduce acceleration (given by the C-metric), NUT charge [59] or introduce boundary gravitons (asymptotic symmetries).

It was shown by Teukolsky [56] that linearized gravitational perturbations of vacuum Petrov type D spacetimes satisfy ${ }^{22}$

$$
\begin{aligned}
& {[(D-3 \epsilon+\bar{\epsilon}-4 \rho-\bar{\rho})(\Delta-4 \gamma+\mu)-} \\
& \left.(\delta+\bar{\varpi}-\bar{\alpha}-3 \beta-4 \tau)(\bar{\delta}+\varpi-4 \alpha)-3 \psi_{2}\right] \delta \psi_{0}=4 \pi \mathcal{T}_{0}, \\
& {[(\Delta+3 \gamma-\bar{\gamma}+4 \mu+\bar{\mu})(D+4 \epsilon-\rho)-} \\
& \left.(\bar{\delta}-\bar{\tau}+\bar{\beta}+3 \alpha+4 \varpi)(\delta-\tau+4 \beta)-3 \psi_{2}\right] \delta \psi_{4}=4 \pi \mathcal{T}_{4},
\end{aligned}
$$

for a Newman-Penrose tetrad with $l^{\mu}, n^{\nu}$ along the two principle null directions. Denoting projections onto the Kinnersley tetrad as $T_{l m}=l^{\mu} m^{\nu} T_{\mu \nu}$, etc, the sources in these

\footnotetext{
${ }^{22}$ The intermediate conventions (metric signature, sign of $\psi_{0}, \psi_{4}$ and sign of spin coefficients) differ from Teukolsky but signs combine so that this final equation is exactly identical 55].
} 
perturbation equations are related to the stress-energy tensor by $\sqrt{2324}$

$$
\begin{aligned}
\mathcal{T}_{0} & =(\delta+\bar{\varpi}-\bar{\alpha}-3 \beta-4 \tau)\left[(D-2 \epsilon-2 \bar{\rho}) T_{l m}-(\delta+\bar{\varpi}-2 \bar{\alpha}-2 \beta) T_{l l}\right] \\
& +(D-3 \epsilon+\bar{\epsilon}-4 \rho-\bar{\rho})\left[(\delta+2 \bar{\varpi}-2 \beta) T_{l m}-(D-2 \epsilon+2 \bar{\epsilon}-\bar{\rho}) T_{m m}\right] \\
\mathcal{T}_{4} & =(\Delta-\bar{\gamma}+\bar{\mu}+3 \gamma+4 \mu)\left[(\bar{\delta}-2 \bar{\tau}+2 \alpha) T_{n \bar{m}}-(\Delta+\bar{\mu}-2 \bar{\gamma}+2 \gamma) T_{\bar{m} \bar{m}}\right] \\
& +(\bar{\delta}+3 \alpha+\bar{\beta}+4 \varpi-\bar{\tau})\left[(\Delta+2 \bar{\mu}+2 \gamma) T_{n \bar{m}}-(\bar{\delta}+2 \alpha+2 \bar{\beta}-\bar{\tau}) T_{n n}\right] .
\end{aligned}
$$

For the Kerr metric in Boyer-Lindquist coordinates $(\hat{t}, \hat{r}, \theta, \hat{\phi})$, the Teukolsky equation [56] for a general spin $s$ field is given by 25

$$
\begin{aligned}
4 \pi \Sigma T_{(s)} & =-\left[\frac{\left(\hat{r}^{2}+a^{2}\right)^{2}}{\Delta}-a^{2} \sin ^{2} \theta\right] \frac{\partial^{2} \Psi_{(s)}}{\partial \hat{t}^{2}}-\frac{4 M a \hat{r}}{\Delta} \frac{\partial^{2} \Psi_{(s)}}{\partial \hat{t} \partial \hat{\phi}} \\
& -2 s\left[\hat{r}-\frac{M\left(\hat{r}^{2}-a^{2}\right)}{\Delta}+i a \cos \theta\right] \frac{\partial \Psi_{(s)}}{\partial \hat{t}}+\Delta^{-s} \frac{\partial}{\partial \hat{r}}\left(\Delta^{s+1} \frac{\partial \Psi_{(s)}}{\partial \hat{r}}\right) \\
& +\frac{1}{\sin \theta} \frac{\partial}{\partial \theta}\left(\sin \theta \frac{\partial \Psi_{(s)}}{\partial \theta}\right)+\left[\frac{1}{\sin ^{2} \theta}-\frac{a^{2}}{\Delta}\right] \frac{\partial^{2} \Psi_{(s)}}{\partial \hat{\phi}^{2}}+2 s\left[\frac{a(\hat{r}-M)}{\Delta}+\frac{i \cos \theta}{\sin ^{2} \theta}\right] \frac{\partial \Psi_{(s)}}{\partial \hat{\phi}} \\
& -\left(s^{2} \cot ^{2} \theta-s\right) \Psi_{(s)} .
\end{aligned}
$$

The equations (A.27)- A.28) precisely reduce to A.31) for the spin $s=-2$ case with $T_{(-2)}=-2 \mathcal{T}_{4} \rho^{-4}$ and $\Psi_{(-2)}=\rho^{-4} \delta \psi_{4}$ and for the spin 2 case with $T_{(2)}=-2 \mathcal{T}_{0}$ and $\Psi_{(2)}=\delta \psi_{0}$. These two scalars contain the complete information about the perturbing field [56]. They are related to each other via the Teukolsky-Starobinsky identity [57] such that it suffices to consider one field to reconstruct the entire metric perturbation. The $s=-2$ field is convenient as it readily relates to asymptotic outgoing gravitational waves.

For the near-NHEK geometry (2.8), the spin -2 Teukolsky equation A.28 reads explicitly as

$$
\begin{aligned}
-8 \pi M^{2}(1 & \left.+\cos ^{2} \theta\right) \eta^{-4} \mathcal{T}_{4}=r(r+2 \kappa) \frac{\partial^{2}\left(\eta^{-4} \delta \psi_{4}\right)}{\partial r^{2}}-2(r+\kappa) \frac{\partial\left(\eta^{-4} \delta \psi_{4}\right)}{\partial r} \\
& -\frac{1}{r(r+2 \kappa)} \frac{\partial^{2}\left(\eta^{-4} \delta \psi_{4}\right)}{\partial t^{2}}+\frac{2(r+\kappa)}{r(r+2 \kappa)} \frac{\partial^{2}\left(\eta^{-4} \delta \psi_{4}\right)}{\partial t \partial \phi}-\frac{4(\kappa+r)}{r(r+2 \kappa)} \frac{\partial\left(\eta^{-4} \delta \psi_{4}\right)}{\partial t} \\
& +\frac{\partial^{2}\left(\eta^{-4} \delta \psi_{4}\right)}{\partial \theta^{2}}+\cot \theta \frac{\partial\left(\eta^{-4} \delta \psi_{4}\right)}{\partial \theta}+\left(-\frac{\kappa^{2}}{r(r+2 \kappa)}+\frac{1}{\sin ^{2} \theta}-2+\frac{\sin ^{2} \theta}{4}\right) \frac{\partial^{2}\left(\eta^{-4} \delta \psi_{4}\right)}{\partial \phi^{2}} \\
& -\left(-\frac{4 \kappa^{2}}{r(r+2 \kappa)}+2 i \cos \theta+\frac{4 i \cos \theta}{\sin ^{2} \theta}\right) \frac{\partial\left(\eta^{-4} \delta \psi_{4}\right)}{\partial \phi}+\left(-\frac{4}{\sin ^{2} \theta}+2\right)\left(\eta^{-4} \delta \psi_{4}\right)
\end{aligned}
$$

\footnotetext{
${ }^{23}$ Note the minus sign typo in front of $\bar{\mu}$ in 28 which is harmless because $\mu=0$ in NHEK.

${ }^{24}$ Compared to $55, \mathcal{T}_{0}$ is their $-T_{+2} /(8 \pi \Sigma)$ defined in (A10) and $\mathcal{T}_{4}$ is their $-\rho^{4} T_{-2} /(8 \pi \Sigma)$ defined in (A11). The identity can be checked using the spin coefficients A.20.

${ }^{25}$ The sign convention for $T_{(s)}$ is defined so that the radial derivative terms have the same sign as the source terms.
} 
with

$$
\eta=-\frac{1}{1-i \cos \theta}
$$

For the NHEK geometry (2.5), equation A.28 reads explicitly as

$$
\begin{aligned}
-8 \pi M^{2}\left(1+\cos ^{2} \theta\right) & \eta^{-4} \mathcal{T}_{4}=R^{2} \frac{\partial^{2}\left(\eta^{-4} \delta \psi_{4}\right)}{\partial R^{2}}-2 R \frac{\partial\left(\eta^{-4} \delta \psi_{4}\right)}{\partial R}-\frac{1}{R^{2}} \frac{\partial^{2}\left(\eta^{-4} \delta \psi_{4}\right)}{\partial T^{2}} \\
& +\frac{2}{R} \frac{\partial^{2}\left(\eta^{-4} \delta \psi_{4}\right)}{\partial T \partial \Phi}-\frac{4}{R} \frac{\partial\left(\eta^{-4} \delta \psi_{4}\right)}{\partial T}+\frac{\partial^{2}\left(\eta^{-4} \delta \psi_{4}\right)}{\partial \theta^{2}}+\cot \theta \frac{\partial\left(\eta^{-4} \delta \psi_{4}\right)}{\partial \theta} \\
& +\left(\frac{1}{\sin ^{2} \theta}-2+\frac{\sin ^{2} \theta}{4}\right) \frac{\partial^{2}\left(\eta^{-4} \delta \psi_{4}\right)}{\partial \Phi^{2}} \\
& -\left(2 i \cos \theta+\frac{4 i \cos \theta}{\sin ^{2} \theta}\right) \frac{\partial\left(\eta^{-4} \delta \psi_{4}\right)}{\partial \Phi}+\left(-\frac{4}{\sin ^{2} \theta}+2\right)\left(\eta^{-4} \delta \psi_{4}\right)
\end{aligned}
$$

where $\eta$ is also defined as A.33.

For a description on how the metric can be reconstructed from $\delta \psi_{4}$ see [23] which expands the original results of 60,61$]$.

\section{A.3 Separation of variables}

Kerr equation The Teukolsky master equation in Kerr A.31) can be separated as

$$
\Psi_{(s)}=\frac{1}{\sqrt{2 \pi}} \int_{-\infty}^{\infty} \mathrm{d} \hat{\omega} \sum_{l m} \hat{R}_{l m \hat{\omega}}(\hat{r}) S_{l m \hat{\omega}}(\theta) e^{i m \hat{\phi}} e^{-i \hat{\omega} \hat{t}}
$$

Here, the spin-weighted spheroidal harmonics $S_{l m \hat{\omega}}(\theta)$ satisfy

$$
\frac{1}{\sin \theta} \frac{\mathrm{d}}{\mathrm{d} \theta}\left(\sin \theta \frac{\mathrm{d} S_{l m}}{\mathrm{~d} \theta}\right)+\left[(a \hat{\omega})^{2} \cos ^{2} \theta-2 a \hat{\omega} s \cos \theta-\left(\frac{m^{2}+2 m s \cos \theta+s^{2}}{\sin ^{2} \theta}\right)+\mathcal{E}_{l m \hat{\omega}}\right] S_{l m \hat{\omega}}=0
$$

where $\mathcal{E}_{l m \hat{\omega}}=\mathcal{E}_{l m \hat{\omega}}[a, s]$ is the separation constant ${ }^{26}$. We have $-l \leq m \leq l$ and $l \geq|s|$. We adopt the convention to keep the dependence in the black hole parameters $M, a$ and the spin $s$ implicit. The spheroidal harmonics are normalized according to

$$
\int_{-1}^{1} d \cos \theta\left(S_{l m \hat{\omega}}(\theta)\right)^{2}=1
$$

The radial equation is given by

$$
\Delta^{-s} \frac{\mathrm{d}}{\mathrm{d} \hat{r}}\left(\Delta^{s+1} \frac{\mathrm{d} \hat{R}_{l m \hat{\omega}}}{\mathrm{d} \hat{r}}\right)-V(\hat{r}) \hat{R}_{l m \hat{\omega}}(\hat{r})=T_{l m \hat{\omega}}(\hat{r})
$$

\footnotetext{
${ }^{26} \operatorname{Our} \mathcal{E}_{l m \omega}$ is $E$ in 62 . The separation constant $\lambda_{s \ell m \omega}$ defined in 55 is also defined in (A.41) in our notation. The separation constant $K$ defined in 22 relates to ours as $K=\mathcal{E}_{l m \hat{\omega}}+\frac{m^{2}}{4}$.
} 
with potential

$$
\begin{aligned}
V(\hat{r}) & =-\frac{\left(K_{m \hat{\omega}}\right)^{2}-2 \operatorname{si}(\hat{r}-M) K_{m \hat{\omega}}}{\Delta}-4 s i \hat{\omega} \hat{r}+\lambda_{\ell m \hat{\omega}} \\
K_{m \hat{\omega}} & \equiv\left(\hat{r}^{2}+a^{2}\right) \hat{\omega}-m a \\
\lambda_{\ell m \hat{\omega}} & \equiv \mathcal{E}_{l m \hat{\omega}}-2 a m \hat{\omega}+a^{2} \hat{\omega}^{2}-s(s+1) .
\end{aligned}
$$

The source of the radial equation is defined as

$$
T_{l m \hat{\omega}}(\hat{r})=\frac{1}{(2 \pi)^{3 / 2}} \int_{-\infty}^{\infty} d \hat{t} \int_{-1}^{1} d \cos \theta \int_{0}^{2 \pi} d \hat{\phi} e^{i(\hat{\omega} t-m \hat{\phi})}(4 \pi \Sigma) T_{(s)} S_{l m \hat{\omega}}(\theta) .
$$

or equivalently one decomposes

$$
(4 \pi \Sigma) T_{(s)}=\frac{1}{\sqrt{2 \pi}} \int_{-\infty}^{\infty} \mathrm{d} \hat{\omega} \sum_{l m} T_{l m \hat{\omega}}(\hat{r}) S_{l m \hat{\omega}}(\theta) e^{i m \hat{\phi}} e^{-i \hat{\omega} \hat{t}} .
$$

near-NHEK equation Let us now turn to Teukolsky's equation $s=-2$ in near-NHEK spacetime A.32. It is separated similarly as

$$
\begin{aligned}
(1-i \cos \theta)^{4} \delta \psi_{4} & =\frac{1}{\sqrt{2 \pi}} \int_{-\infty}^{\infty} \mathrm{d} \omega \sum_{l m} R_{l m \omega}(r) S_{l m}(\theta) e^{i m \phi} e^{-i \omega t} \\
-8 \pi M^{2}\left(1+\cos ^{2} \theta\right) \eta^{-4} \mathcal{T}_{4} & =\frac{1}{\sqrt{2 \pi}} \int_{-\infty}^{\infty} \mathrm{d} \omega \sum_{l m} T_{l m \omega}(r) S_{l m}(\theta) e^{i m \phi} e^{-i \omega t}
\end{aligned}
$$

The $s=2$ equation can be separated similarly but we will omit the explicit formulae. The extremal spheroidal harmonics $S_{l m}(\theta)$ are now defined from A.36 as

$$
S_{l m}(\theta)=\left.S_{l m \hat{\omega}}(\theta)\right|_{\hat{\omega}=\frac{m}{2 a}}
$$

where the separation constant can now be written as $\mathcal{E}_{l m}$ instead of $\mathcal{E}_{\text {lmä }}{ }^{28}$, namely

$$
\frac{1}{\sin \theta} \frac{\mathrm{d}}{\mathrm{d} \theta}\left(\sin \theta \frac{\mathrm{d} S_{l m}}{\mathrm{~d} \theta}\right)+\left[\frac{m^{2}}{4} \cos ^{2} \theta-m s \cos \theta-\left(\frac{m^{2}+2 m s \cos \theta+s^{2}}{\sin ^{2} \theta}\right)+\mathcal{E}_{l m}\right] S_{l m}=0 .
$$

Only these specific harmonics occur because the NHEK spacetime is spanned only by corotating modes $\left(\hat{\omega}=\Omega_{e x t} m=\frac{m}{2 a}\right)$ up to small deviations. For $m$ small and any $s$ we

\footnotetext{
${ }^{27}$ The extremal spheroidal harmonics for $s=0$ are built in Mathematica as SpheroidalPS. The $s=-2$ case can be solved using the spectral decomposition method, see appendix A of [63]. A online package is available on 64 based on the resummation method outlined in 65.

${ }^{28}$ The separation constant $K_{\ell}$ for $S_{l m}(\theta)$ defined in 28 relates to ours as $K_{\ell}=\mathcal{E}_{l m}+\frac{m^{2}}{4}$. The separation constant $\Lambda_{l m}^{(s)}$ defined in 23 . relates to ours as $\Lambda_{l m}^{(s)}=\mathcal{E}_{l m}-s(s+1)$. Note that $\mathcal{E}_{l m}=\frac{7 m^{2}}{4}+h(h-1)$ where $h$ is defined in 4.10.
} 
have $\mathcal{E}_{l m}=l(l+1)+O(m)$. The equation is invariant under $\theta \mapsto \pi-\theta$ together with either $m \mapsto-m$ or $s \mapsto-s$. We therefore have $\mathcal{E}_{l m}^{(-s)}=\mathcal{E}_{l m}^{(s)}=\mathcal{E}_{l,-m}^{(s)}$. The radial separation function now satisfies the $s=-2$ or $s=2$ case of

$$
(r(r+2 \kappa))^{-s} \frac{\mathrm{d}}{\mathrm{d} r}\left((r(r+2 \kappa))^{s+1} \frac{\mathrm{d} R_{l m \omega}}{\mathrm{d} r}\right)-V(r) R_{l m \omega}(r)=T_{l m \omega}(r)
$$

with potential 29

$$
V(r)=-\frac{3}{4} m^{2}-s(s+1)+\mathcal{E}_{l m}-2 i s m+\frac{(m r+\kappa n)(\kappa(2 s i-n)+r(2 s i-m))}{r(r+2 \kappa)}
$$

where

$$
n=m+\frac{\omega}{\kappa}
$$

NHEK equation Let us finally turn to Teukolsky's equation $s=-2$ in NHEK spacetime A.34. It is separated similarly as

$$
\begin{aligned}
(1-i \cos \theta)^{4} \delta \psi_{4} & =\frac{1}{\sqrt{2 \pi}} \int_{-\infty}^{\infty} \mathrm{d} \Omega \sum_{l m} R_{l m \Omega}(R) S_{l m}(\theta) e^{i m \Phi} e^{-i \Omega T} \\
-8 \pi M^{2}\left(1+\cos ^{2} \theta\right) \eta^{-4} \mathcal{T}_{4} & =\frac{1}{\sqrt{2 \pi}} \int_{-\infty}^{\infty} \mathrm{d} \Omega \sum_{l m} T_{l m \Omega}(R) S_{l m}(\theta) e^{i m \Phi} e^{-i \Omega T}
\end{aligned}
$$

The spheroidal harmonics are defined as A.46). The radial separation function now satisfies the $s=-2$ case of

$$
R^{-2 s} \frac{\mathrm{d}}{\mathrm{d} R}\left(R^{2 s+2} \frac{\mathrm{d} R_{l m \Omega}}{\mathrm{d} R}\right)-V(R) R_{l m \Omega}(R)=T_{l m \Omega}(R)
$$

with potentia ${ }^{30}$

$$
V(R)=-\frac{7}{4} m^{2}+\mathcal{E}_{l m}-s(s+1)-\frac{2 \Omega(m-i s)}{R}-\frac{\Omega^{2}}{R^{2}}
$$

Formally, the NHEK equation is obtained from the near-NHEK equation upon substituting $(r, t, \phi) \mapsto(R, T, \Phi)$ and setting $\kappa=0$.

\footnotetext{
${ }^{29}$ Note that our convention for $V(r)$ differs from 28 by an overall minus sign, so that it has the interpretation of a standard potential.

${ }^{30}$ Our sign convention for $V(R)$ is again the opposite of the one chosen in 28 .
} 


\section{B Detailed taxonomy of (near-)NHEK orbits}

\section{B.1 Timelike NHEK orbits}

B.1.1 $\ell=\ell_{*}, E=0$ Circular $_{*}($ ISCO $)$

$$
\begin{aligned}
R & =R_{0}, \\
\Phi & =\Phi_{0}-\frac{3}{4} R_{0} T
\end{aligned}
$$

where $R_{0}>0$ and $\Phi_{0} \in[0,2 \pi]$.

B.1.2 $\ell=\ell_{*}, E>0$ Plunging $*(E)$

$$
\begin{aligned}
& T=T_{0}+\frac{1}{R} \sqrt{1+\frac{2 \ell_{*} R}{E}}, \\
& \Phi=\Phi_{0}+\frac{3}{4} \sqrt{1+\frac{2 \ell_{*} R}{E}}-2 \operatorname{arctanh} \sqrt{1+\frac{2 \ell_{*} R}{E}}
\end{aligned}
$$

where $T_{0} \in \mathbb{R}$ and $\Phi_{0} \in[0,2 \pi]$.

B.1.3 $\ell>\ell_{*}, E>0$ Plunging $(E, \ell)$ or $E<0$ Osculating $(E, \ell)$

$$
T=T_{0}+\frac{F(R)}{2 E R}
$$

$\Phi=\Phi_{0}-\log \frac{2 E+2 \ell R+F(R)}{R}+\frac{\sqrt{3} \ell}{2 \sqrt{\ell^{2}-\ell_{*}^{2}}} \log \left(\sqrt{3\left(\ell^{2}-\ell_{*}^{2}\right)} F(R)+3 R\left(\ell^{2}-\ell_{*}^{2}\right)+4 E \ell\right)$

where $F(r)=\sqrt{4 E^{2}+8 E \ell R+3\left(\ell^{2}-\ell_{*}^{2}\right) R^{2}}$.

B.1.4 $\ell>\ell_{*}, E=0 \operatorname{Marginal}(\ell)$

$$
\begin{aligned}
T & =T_{0}+\frac{2 \ell}{\sqrt{3\left(\ell^{2}-\ell_{*}^{2}\right)}}, \\
\Phi & =\Phi_{0}+\frac{\sqrt{3 \ell}}{2 \sqrt{\ell^{2}-\ell_{*}^{2}}} \log R .
\end{aligned}
$$

It is marginally plunging or, equivalently, marginally osculating. 


\section{B.2 Timelike near-NHEK orbits}

B.2.1 $\ell=\ell_{*}, e=0$ Plunging $(e=0)$

$$
\begin{aligned}
t & =t_{0}-\frac{1}{2 \kappa} \log (r(r+2 \kappa)), \\
\phi & =\phi_{0}+\frac{3}{4 \kappa} r-\frac{1}{2} \log \left(1+\frac{2 \kappa}{r}\right) .
\end{aligned}
$$

B.2.2 $\ell=\ell_{*}, e>0$ Plunging ${ }_{*}(e)$

$$
\begin{aligned}
t & =t_{0}+\frac{1}{\kappa} \operatorname{arccosh} \frac{r+\kappa\left(1+\frac{\kappa \ell^{*}}{e}\right)}{\sqrt{r(r+2 \kappa)}} \\
\phi & =\phi_{0}+\frac{3}{4 e} F-\frac{1}{2} \log \left(1+\frac{2 \kappa}{r}\right)+\log \frac{F+\ell_{*} \kappa-e}{F+\ell^{*} \kappa+e}
\end{aligned}
$$

where $F=\sqrt{\left(e+\ell_{*} \kappa\right)^{2}+2 \ell_{*} e r}$. Note that for $e>0$, the argument of the arccosh is bigger than 1 and therefore $t$ is real.

Note that for $e=\kappa \ell^{*}$ the orbit reduces to

$$
\begin{aligned}
t & =t_{0}+\frac{1}{2 \kappa} \log \frac{\sqrt{1+\frac{r}{2 \kappa}}+1}{\sqrt{1+\frac{r}{2 \kappa}}-1} \\
\phi & =\phi_{0}+\frac{3}{2} \sqrt{1+\frac{r}{2 \kappa}}-\frac{1}{2} \log \frac{\sqrt{1+\frac{r}{2 \kappa}}+1}{\sqrt{1+\frac{r}{2 \kappa}}-1} .
\end{aligned}
$$

B.2.3 $\ell>\ell_{*}, e=-\frac{\sqrt{3} \kappa}{2} \sqrt{\ell^{2}-\ell_{*}^{2}} \operatorname{Circular}(\ell)$

$$
\begin{aligned}
r & =r_{0}=\frac{2 \kappa \ell}{\sqrt{3\left(\ell^{2}-\ell_{*}^{2}\right)}}-\kappa, \\
\phi & =\phi_{0}-\frac{3}{4}\left(r_{0}+\kappa\right) t .
\end{aligned}
$$

Note that $r_{0} \geq\left(\frac{2}{\sqrt{3}}-1\right) \kappa$.

\section{B.2.4 $\ell>\ell_{*}$, Osculating $(e, \ell)$ or Plunging $(e, \ell)$}

$$
\begin{aligned}
t= & t_{0}-\frac{1}{2 \kappa} \log \left(1+\frac{2 \kappa}{r}\right)+\frac{1}{2 \kappa} \log \frac{\left(3 \kappa \ell^{2}-4 \kappa M^{2}-4 e \ell\right) r+2\left(\kappa^{2} \ell^{2}-2 e^{2}-4 \kappa^{2} M^{2}+(e-\kappa \ell) F\right)}{\left(3 \kappa \ell^{2}-4 \kappa M^{2}+4 e \ell\right) r+4(e+\kappa \ell)^{2}-2(e+\kappa \ell) F} \\
\phi= & \phi_{0}-\frac{1}{2} \log (r(r+2 \kappa))+\frac{\sqrt{3} \ell}{2 \sqrt{\ell^{2}-\ell_{*}^{2}}} \log \left(3\left(\ell^{2}-\ell_{*}^{2}\right)(r+\kappa)+\sqrt{3\left(\ell^{2}-\ell_{*}^{2}\right)} F+4 e \ell\right) \\
& +\frac{1}{2} \log \left(\left(7 \ell^{2}-4 M^{2}\right) r(r+2 \kappa)+16 e \ell r+8(e+\ell \kappa)^{2}-4(e+\ell(r+\kappa)) F\right)
\end{aligned}
$$


where $F=\sqrt{3\left(\ell^{2}-\ell_{*}^{2}\right) r(r+2 \kappa)+8 e \ell r+4(e+\kappa \ell)^{2}}$.

For $e<-\frac{\sqrt{3} \kappa}{2} \sqrt{\ell^{2}-\ell_{*}^{2}}$ the orbits are osculating. For $e>-\frac{\sqrt{3} \kappa}{2} \sqrt{\ell^{2}-\ell_{*}^{2}}$ they are plunging. For $e=-\frac{\sqrt{3} \kappa}{2} \sqrt{\ell^{2}-\ell_{*}^{2}}$ the orbits are circular and given by (B.14). These orbits were considered in 31 .

\section{B.2.5 $\ell>\ell_{*}, e=-\kappa \ell$ Second Circular $(\ell)$}

$$
\begin{aligned}
& r=r_{0}=\frac{2 \kappa\left(\ell^{2}+4 M^{2}\right)}{3\left(\ell^{2}-\ell_{*}^{2}\right)}, \\
& \phi=\phi_{0}-\frac{3 \ell^{2}+4 M^{2}}{3\left(\ell^{2}-\ell_{*}^{2}\right)} \kappa t .
\end{aligned}
$$

Note that $r_{0} \geq \frac{2}{3} \kappa$.

\section{B.3 Conformal transformations}

First, the inverse transformation of (2.11) defined for $R>0,1+R^{2}\left(1-T^{2}\right)>0$ is given by

$$
\begin{aligned}
y & =\frac{R^{2}\left(1+T^{2}\right)-1}{2 R}, \\
\tau & =\arctan \frac{2 R^{2} T}{1+R^{2}\left(1-T^{2}\right)}, \\
\varphi & =\Phi+\log \frac{R^{2}+(1+R T)^{2}}{\sqrt{1+2 R^{2}\left(1-T^{2}\right)+R^{4}\left(1+T^{2}\right)^{2}}} .
\end{aligned}
$$

We can then deduce that under a global translation $\tau \rightarrow \bar{\tau}=\tau+\zeta$, the Poincaré coordinates $(R, T, \Phi)$ are transformed to new Poincaré coordinates $(\bar{R}, \bar{T}, \bar{\Phi})$ as

$$
\begin{aligned}
\bar{R} & =\frac{R^{2}\left(1+T^{2}\right)-1+\left(1+R^{2}\left(1-T^{2}\right)\right) \cos \zeta-2 R^{2} T \sin \zeta}{2 R}, \\
\bar{T} & =\frac{2 R^{2} T \cos \zeta+\left(1+R^{2}\left(1-T^{2}\right)\right) \sin \zeta}{2 R} \frac{1}{\bar{R}}, \\
\bar{\Phi} & =\Phi+\log \frac{\cos \frac{\zeta}{2} R-\sin \frac{\zeta}{2}(1+R T)}{\cos \frac{\zeta}{2} R-\sin \frac{\zeta}{2}(-1+R T)} .
\end{aligned}
$$

The inverse coordinate transformation of (2.10) defined for $R>0$ and $R T<-1$ is 
given by

$$
\begin{aligned}
r & =\kappa(-R T-1) \\
t & =\frac{1}{\kappa} \log \frac{R}{\sqrt{R^{2} T^{2}-1}}, \\
\phi & =\Phi+\frac{1}{2} \log \frac{-R T-1}{-R T+1} .
\end{aligned}
$$

We now define

$$
\begin{aligned}
\bar{r} & =\kappa(-\bar{R} \bar{T}-1) \\
\bar{t} & =\frac{1}{\kappa} \log \frac{\bar{R}}{\sqrt{\bar{R}^{2} \bar{T}^{2}-1}}, \\
\bar{\phi} & =\bar{\Phi}+\frac{1}{2} \log \frac{-\bar{R} \bar{T}-1}{-\bar{R} \bar{T}+1} .
\end{aligned}
$$

Substituting (B.19) and 2.10) we obtain the transformation from near-NHEK to nearNHEK coordinates which corresponds to a global time translation $\tau \rightarrow \bar{\tau}=\tau+\zeta$ as

$$
\begin{aligned}
r & =(\bar{r}+\kappa) \cos \zeta-\sqrt{\bar{r}(\bar{r}+2 \kappa)} \sin \zeta \sinh \kappa \bar{t}-\kappa, \\
t & =\frac{1}{2 \kappa} \log \frac{\sqrt{\bar{r}(\bar{r}+2 \kappa)}(\cosh \kappa \bar{t}+\cos \zeta \sinh \kappa \bar{t})+\sin \zeta(\bar{r}+\kappa)}{\sqrt{\bar{r}(\bar{r}+2 \kappa)}(\cosh \kappa \bar{t}-\cos \zeta \sinh \kappa \bar{t})-\sin \zeta(\bar{r}+\kappa)}, \\
\phi & =\bar{\phi}+\frac{1}{2} \log \frac{r}{r+2 \kappa}+\log \frac{e^{\kappa \bar{t}} \sqrt{\bar{r}+2 \kappa} \cos \frac{\zeta}{2}+\sqrt{\bar{r}} \sin \frac{\zeta}{2}}{e^{\kappa \bar{t}} \sqrt{\bar{r}} \cos \frac{\zeta}{2}+\sqrt{\bar{r}+2 \kappa} \sin \frac{\zeta}{2}}
\end{aligned}
$$

The two near-NHEK patches admit an overlap in the range $-\frac{\pi}{2}<\zeta<\frac{\pi}{2}$. For $\zeta=-\pi / 2$, (B.22) reduces to the change of coordinates of 31 for $\chi=0$,

$$
\begin{aligned}
r & =\sqrt{\bar{r}(\bar{r}+2 \kappa)} \sinh \kappa \bar{t}-\kappa, \\
t & =\frac{1}{2 \kappa} \log \frac{\sqrt{\bar{r}(\bar{r}+2 \kappa)} \cosh \kappa \bar{t}-(\bar{r}+\kappa)}{\sqrt{\bar{r}(\bar{r}+2 \kappa)} \cosh \kappa \bar{t}+(\bar{r}+\kappa)}, \\
\phi & =\bar{\phi}+\frac{1}{2} \log \frac{(\bar{r}+\kappa) \sinh \kappa \bar{t}+\kappa \cosh \kappa \bar{t}}{(\bar{r}+\kappa) \sinh \kappa \bar{t}-\kappa \cosh \kappa \bar{t}} .
\end{aligned}
$$

In the following we will enumerate the conformal transformations relating the Circular $*$ (ISCO) orbit (B.2) of NHEK to either NHEK or near-NHEK orbits, and the conformal transformations relating the Circular $(\ell)$ orbit (B.14) of near-NHEK to either NHEK or near-NHEK orbits. 


\section{B.3.1 ISCO to NHEK orbits}

- $\operatorname{Circular}_{*}(\mathrm{ISCO}) \Leftrightarrow \operatorname{Plunging}_{*}(E)$.

We denote the initial NHEK coordinates as $(T, R, \Phi)$ and the new NHEK coordinates as $(\bar{T}, \bar{R}, \bar{\Phi})$. We can map the $R T \leq-1$ patch to the $\bar{R} \bar{T} \geq 1$ patch via (see [30])

$$
\begin{aligned}
T & =-\frac{\bar{R}^{2} \bar{T}}{\bar{R}^{2} \bar{T}^{2}-1}, \\
R & =\frac{\bar{R}^{2} \bar{T}^{2}-1}{\bar{R}}, \\
\Phi & =\bar{\Phi}+\log \frac{\bar{R} \bar{T}+1}{\bar{R} \bar{T}-1} .
\end{aligned}
$$

The Circular ${ }_{*}$ orbit is then mapped to the $\operatorname{Plunging}_{*}(E)$ orbit with parameters

$$
\begin{aligned}
E & =\frac{2 \ell_{*}}{R_{0}}, \quad \ell=\ell^{*}, \\
\bar{T}_{0} & =0, \quad \bar{\Phi}_{0}=\Phi_{0}-i \pi .
\end{aligned}
$$

It is then necessary to perform the imaginary shift

$$
\bar{\Phi} \rightarrow \bar{\Phi}+i \pi \text {. }
$$

Generic orbits are obtained after the shift $\bar{T} \rightarrow \bar{T}+\bar{T}_{0}$. The two orbits are therefore related by a complex $S L(2, \mathbb{R}) \times U(1)$ transformation.

\section{B.3.2 ISCO to near-NHEK orbits}

- Circular $_{*}(\mathrm{ISCO}) \Leftrightarrow \operatorname{Plunging}_{*}(e=0)$.

These orbits are simply related by the defining change of coordinates between the NHEK patch and the near-NHEK patch 2.10$)$. The parameters of the orbits are related as

$$
R_{0}=\frac{1}{\kappa} e^{\kappa t_{0}}, \quad \Phi_{0}=\phi_{0}-\frac{3}{4}
$$

This parametrizes a generic orbit.

- Circular $_{*}(\mathrm{ISCO}) \Leftrightarrow$ Plunging $_{*}(e)$.

We can reach the orbit (B.10) (with barred coordinates) starting from the orbit Plunging $_{*}(e=0)$ (with unbarred coordinates) by applying a shift as $\tau \rightarrow \bar{\tau}=\tau+\zeta$ followed by a near-NHEK time shift $\bar{t} \rightarrow \bar{t}-\frac{i \pi}{\kappa}$. 
The diffeomorphism between two near-NHEK patches of same parameter $\kappa$ which is exactly a $\tau \rightarrow \bar{\tau}=\tau+\zeta$ translation is given by (B.22). We then obtain the orbit (B.10) (with barred coordinates) with $0<\zeta<\pi$ and

$$
\begin{aligned}
e & =\kappa^{2} \ell_{*} \sin \zeta e^{-\kappa t_{0}}, \quad \ell=\ell_{*}, \\
\bar{t}_{0} & =\frac{i \pi}{\kappa}+\frac{1}{\kappa} \log \left(\frac{\sin \zeta}{1+\cos \zeta}\right), \quad \bar{\phi}_{0}=\phi_{0}-\frac{3}{4}\left(\frac{\cot \zeta}{\kappa} e^{\kappa t_{0}}+1\right) .
\end{aligned}
$$

It is then necessary to perform an imaginary time shift $\bar{t} \rightarrow \bar{t}-\frac{i \pi}{\kappa}$. All energies can be reached by using simply $\zeta= \pm \frac{\pi}{2}$ (for $\zeta<0$ the energy $e$ is positive after an initial time shift $\left.t_{0} \rightarrow t_{0}+\frac{i \pi}{\kappa}\right)$.

\section{B.3.3 near-NHEK circular to NHEK orbits}

- $\operatorname{Circular}(\ell) \Leftrightarrow \operatorname{Marginal}(\ell)$

We start from the Circular $(\ell)$ orbits. We first apply the inverse coordinate transformation of 2.10 given in (B.20). We then apply the $P T$ flip $T \rightarrow-T$ and $\Phi \rightarrow-\Phi$. We obtain the Marginal $(\ell)$ orbits (B.6) with parameters

$$
\begin{aligned}
E & =0, \quad \ell>\ell_{*}, \quad T_{0}=0 \\
\Phi_{0} & =-\phi_{0}+\frac{3 \log \kappa}{4 \kappa}\left(r_{0}+\kappa\right)+\frac{1}{2} \log \frac{r_{0}}{r_{0}+2 \kappa}-\frac{3\left(r_{0}+\kappa\right)}{8 \kappa} \log \left(r_{0}\left(r_{0}+2 \kappa\right)\right) .
\end{aligned}
$$

We can always shift $T_{0}$ to be nonzero.

- $\operatorname{Marginal}(\ell) \Leftrightarrow \operatorname{Plunging}(E, \ell)$ or $\operatorname{Osculating}(E, \ell)$

Starting from the Marginal $(\ell)$ orbit (B.6), we can apply the shift $\tau \rightarrow \bar{\tau}=\tau+\zeta$ expressed as a NHEK to NHEK diffeomorphism (B.19) or, equivalently,

$$
\begin{aligned}
R & =\frac{\bar{R}^{2}\left(1+\bar{T}^{2}\right)-1+\left(1+\bar{R}^{2}\left(1-\bar{T}^{2}\right)\right) \cos \zeta+2 \bar{R}^{2} \bar{T} \sin \zeta}{2 \bar{R}}, \\
T & =\frac{2 \bar{R}^{2} \bar{T} \cos \zeta-\left(1+\bar{R}^{2}\left(1-\bar{T}^{2}\right)\right) \sin \zeta}{2 \bar{R}} \frac{1}{R}, \\
\Phi & =\bar{\Phi}+\log \frac{\cos \frac{\zeta}{2} \bar{R}+\sin \frac{\zeta}{2}(1+\bar{R} \bar{T})}{\cos \frac{\zeta}{2} \bar{R}+\sin \frac{\zeta}{2}(-1+\bar{R} \bar{T})} .
\end{aligned}
$$

Assuming $\zeta \neq 0$, we obtain the $\operatorname{Plunging}(E, \ell)$ or $\operatorname{Osculating}(E, \ell)$ orbits in barred 
coordinates with

$$
\begin{aligned}
E & =\frac{\sqrt{3\left(\ell^{2}-\ell_{*}^{2}\right)}}{2}\left(\sin \zeta+T_{0}(\cos \zeta-1)\right), \quad \ell>\ell_{*}, \\
\bar{T}_{0} & =\frac{-\cos \zeta+T_{0} \sin \zeta}{\sin \zeta+T_{0}(\cos \zeta-1)}, \\
\bar{\Phi}_{0} & =\Phi_{0}+\log \left(2 \ell+\sqrt{3\left(\ell^{2}-\ell_{*}^{2}\right)}\right)-\frac{\sqrt{3} \ell}{2 \sqrt{\ell^{2}-\ell_{*}^{2}}} \log \left(6\left(\ell^{2}-\ell_{*}^{2}\right)\left(\cos \frac{\zeta}{2}-T_{0} \sin \frac{\zeta}{2}\right)^{2}\right) .
\end{aligned}
$$

\section{B.3.4 near-NHEK circular to near-NHEK orbits}

- Circular $(\ell) \Leftrightarrow$ Osculating $(e, \ell)$ or Plunging $(e, \ell)$

We consider a $T \rightarrow T-\chi$ translation followed by a $\tau \rightarrow \tau-\frac{\pi}{2}$ translation. It leads to another near-NHEK patch $(\bar{t}, \bar{r}, \bar{\phi})$ given by (see also 31 )

$$
\begin{aligned}
r & =\sqrt{\bar{r}(\bar{r}+2 \kappa)}(\sinh \kappa \bar{t}+\chi \cosh \kappa \bar{t})-\chi(\bar{r}+\kappa)-\kappa \\
t & =\frac{1}{\kappa} \log \frac{\sqrt{\bar{r}(\bar{r}+2 \kappa)} \cosh \kappa \bar{t}-(\bar{r}+\kappa)}{\sqrt{r(r+2 \kappa)}}, \\
\phi & =\bar{\phi}-\frac{1}{2} \log \frac{\sqrt{\bar{r}(\bar{r}+2 \kappa)}-(\bar{r}+\kappa) \cosh \kappa \bar{t}+\kappa \sinh \kappa \bar{t}}{\sqrt{\bar{r}(\bar{r}+2 \kappa)}-(\bar{r}+\kappa) \cosh \kappa \bar{t}-\kappa \sinh \kappa \bar{t}} \frac{r}{r} .
\end{aligned}
$$

Using this diffeomorphism with $\chi \neq \pm 1$ we can map the near-NHEK circular orbits B.14 to the near-NHEK Osculating $(e, \ell)$ or Plunging $(e, \ell)$ orbits B.15) with

$$
\begin{aligned}
e & =\frac{1}{2} \sqrt{3\left(\ell^{2}-\ell_{*}^{2}\right)} \kappa \chi, \quad \ell>\ell_{*} \\
\bar{t}_{0} & =-\frac{1}{2 \kappa} \log \frac{1+\chi}{1-\chi} \\
\bar{\phi}_{0} & =\phi_{0}-\frac{1}{2} \log \left(\ell^{2}+4 M^{2}\right)-\frac{\sqrt{3} \ell}{4 \sqrt{\ell^{2}-\ell_{*}^{2}}} \log \left(3\left(\ell^{2}-\ell_{*}^{2}\right) \kappa^{2}\left(\ell^{2}+4 M^{2}\right)\right) .
\end{aligned}
$$

For $-1<\chi<1, \bar{t}_{0}$ is real, but for $|\chi|>1, \bar{t}_{0}$ is complex, and therefore a complex shift of $\bar{t}$ is required as a final step.

Alternatively, one can use the diffeomorphism $(\mathrm{B} .22)$ with $\zeta<0$ to map near-NHEK circular orbits (B.14) to near-NHEK Osculating $(e, \ell)$ or Plunging $(e, \ell)$ orbits (B.15) with

$$
\begin{aligned}
\frac{e}{\kappa} & =-\frac{1}{2} \sqrt{3\left(\ell^{2}-\ell_{*}^{2}\right)} \cos \zeta, \quad \ell>\ell_{*}, \\
\overline{t_{0}} & =0
\end{aligned}
$$

However, this map is limited to the range $|e|<\frac{1}{2} \sqrt{3\left(\ell^{2}-\ell_{*}^{2}\right)} \kappa$. 


\section{Emission from the ISCO orbit in NHEK}

The circular orbit is given in $(\mathrm{B} .2)$ where we set $\Phi_{0}=0$. The covariant components of the stress-tensor (4.6) are then vanishing except for

$$
T_{\Phi \Phi}=\frac{m_{0} R_{0}}{\sqrt{3} M} \delta\left(R-R_{0}\right) \delta\left(\theta-\frac{\pi}{2}\right) \delta(\Phi+\tilde{\Omega} T)
$$

where

$$
\tilde{\Omega}=-\frac{3 R_{0}}{4} .
$$

From the NHEK limit (2.7), the ISCO is located at $R_{0}=2^{1 / 3}$. As a result, only the frequency $\Omega=m \tilde{\Omega}$ will appear in the source A.52 and therefore also in the curvature perturbation A.51 which then simplify to

$$
\begin{aligned}
\delta \psi_{4} & =\frac{1}{(1-i \cos \theta)^{4}} \sum_{l m} R_{l m \tilde{\Omega}}(R) S_{l m}(\theta) e^{i m(\Phi-\tilde{\Omega} T)} \\
4 \pi \mathcal{T}_{4} & =-\frac{1}{2 M^{2}\left(1+\cos ^{2} \theta\right)(1-i \cos \theta)^{4}} \sum_{l, m} T_{l m \tilde{\Omega}}(R) S_{l m}(\theta) e^{i m(\Phi-\tilde{\Omega} T)}
\end{aligned}
$$

The source term $\mathcal{T}_{4}$ is a particular combination of differential operators acting on projections of the stress-energy tensor. The general expression is given in A.30) in Appendix A. We find the same result as [28]:

$$
\begin{aligned}
\mathcal{T}_{4} & =\frac{m_{0} R_{0}^{3}}{64 \sqrt{3} M^{7}}\left\{144 \delta\left(R-R_{0}\right) \delta\left(\theta-\frac{\pi}{2}\right) \delta(\Phi-\tilde{\Omega} T)+16 R_{0} \delta^{\prime}\left(R-R_{0}\right) \delta\left(\theta-\frac{\pi}{2}\right) \delta(\Phi-\tilde{\Omega} T)\right. \\
& -48 i \delta\left(R-R_{0}\right) \delta^{\prime}\left(\theta-\frac{\pi}{2}\right) \delta(\Phi-\tilde{\Omega} T)-21 \delta\left(R-R_{0}\right) \delta\left(\theta-\frac{\pi}{2}\right) \delta^{\prime}(\Phi-\tilde{\Omega} T) \\
& -8 i R_{0} \delta^{\prime}\left(R-R_{0}\right) \delta^{\prime}\left(\theta-\frac{\pi}{2}\right) \delta(\Phi-\tilde{\Omega} T)-3 R_{0} \delta^{\prime}\left(R-R_{0}\right) \delta\left(\theta-\frac{\pi}{2}\right) \delta^{\prime}(\Phi-\tilde{\Omega} T) \\
& +6 i \delta\left(R-R_{0}\right) \delta^{\prime}\left(\theta-\frac{\pi}{2}\right) \delta^{\prime}(\Phi-\tilde{\Omega} T)+2 R_{0}^{2} \delta^{\prime \prime}\left(R-R_{0}\right) \delta\left(\theta-\frac{\pi}{2}\right) \delta(\Phi-\tilde{\Omega} T) \\
& \left.-8 \delta\left(R-R_{0}\right) \delta^{\prime \prime}\left(\theta-\frac{\pi}{2}\right) \delta(\phi-\tilde{\Omega} T)+\frac{9}{8} \delta\left(R-R_{0}\right) \delta\left(\theta-\frac{\pi}{2}\right) \delta^{\prime \prime}(\Phi-\tilde{\Omega} T)\right\} .
\end{aligned}
$$

The expression can be written more conveniently as a special case of the general formula

$$
\mathcal{T}_{4}=\frac{m_{0} R_{0}^{3}}{M^{7}} \times \sum_{\substack{\{i, j, k \in \mathbb{N} \mid \\ i+j+k \leq 2\}}} a_{i j k} R_{0}^{i} \delta^{(i)}\left(R-R_{0}\right) \delta^{(j)}\left(\theta-\frac{\pi}{2}\right) \delta^{(k)}(\Phi-\tilde{\Omega} T)
$$


where $a_{i j k}$ are pure numbers. Using the convention A.37), the radial function $T_{l m \tilde{\Omega}}(R)$ is defined from (C.4) as

$$
T_{l m \tilde{\Omega}}(R)=-4 M^{2} \int_{0}^{2 \pi} d \Phi e^{-i m \Phi} \int_{-1}^{1} d \cos \theta S_{l m}(\theta)\left(1+\cos ^{2} \theta\right)(1-i \cos \theta)^{4} \mathcal{T}_{4} .
$$

For the generic form (C.6) we find

$$
\begin{aligned}
T_{l m \tilde{\Omega}} & =-\frac{4 m_{0} R_{0}^{3}}{M^{5}}\left\{\delta\left(R-R_{0}\right)\left[\mathcal{S}\left(a_{000}+i m a_{001}-m^{2} a_{002}\right)-\mathcal{S}^{\prime}\left(a_{010}+i m a_{011}\right)+\mathcal{S}^{\prime \prime}\left(a_{020}\right)\right]\right. \\
& \left.+R_{0} \delta^{\prime}\left(R-R_{0}\right)\left[\mathcal{S}\left(a_{100}+i m a_{101}\right)-\mathcal{S}^{\prime}\left(a_{110}\right)\right]+R_{0}^{2} \delta^{\prime \prime}\left(R-R_{0}\right)\left[\mathcal{S}\left(a_{200}\right)\right]\right\}
\end{aligned}
$$

where

$$
\begin{aligned}
\mathcal{S} & =S_{l m}(\pi / 2) \\
\mathcal{S}^{\prime} & =S_{l m}^{\prime}(\pi / 2)+4 i S_{l m}(\pi / 2) \\
\mathcal{S}^{\prime \prime} & =S_{l m}^{\prime \prime}(\pi / 2)+8 i S_{l m}^{\prime}(\pi / 2)-11 S_{l m}(\pi / 2)
\end{aligned}
$$

and ' is the derivative with respect to $\theta$. In the case of (C.5), we find

$$
T_{l m \tilde{\Omega}}(R)=a_{0} \delta\left(R-R_{0}\right)+a_{1} R_{0} \delta^{\prime}\left(R-R_{0}\right)+a_{2} R_{0}^{2} \delta^{\prime \prime}\left(R-R_{0}\right)
$$

with

$$
\begin{aligned}
& a_{0}=-\frac{m_{0} R_{0}^{3}}{16 \sqrt{3} M^{5}}\left[\left(40+3 i m-\frac{9}{8} m^{2}\right) S_{l m}\left(\frac{\pi}{2}\right)+(6 m-16 i) S_{l m}^{\prime}\left(\frac{\pi}{2}\right)-8 S_{l m}^{\prime \prime}\left(\frac{\pi}{2}\right)\right] \\
& a_{1}=-\frac{m_{0} R_{0}^{3}}{16 \sqrt{3} M^{5}}\left[(-3 i m-16) S_{l m}\left(\frac{\pi}{2}\right)+8 i S_{l m}^{\prime}\left(\frac{\pi}{2}\right)\right] \\
& a_{2}=-\frac{m_{0} R_{0}^{3}}{8 \sqrt{3} M^{5}} S_{l m}\left(\frac{\pi}{2}\right)
\end{aligned}
$$

in agreement with [28] up to the global sign which differs 31 .

Following Green's function methods, the general solution which is ingoing at the horizon and sourced by $\mathcal{T}_{4}$ is given by

$$
R_{l m \tilde{\Omega}}(R)=\frac{R_{0}^{2 s}}{W}\left(\mathcal{X} \Theta\left(R_{0}-R\right) \mathcal{W}^{\text {in }}(R)+\mathcal{Z} \Theta\left(R-R_{0}\right) \mathcal{M}^{\mathrm{D}}(R)+\mathcal{Y} \mathcal{W}^{\text {in }}(R)\right)+a_{2} \delta\left(R-R_{0}\right)
$$

\footnotetext{
${ }^{31}$ The global sign difference originates from the sign of the stress-tensor in Teukolsky equations (A.31). This global sign difference leads to phase shift which does not modify the amplitude or energy fluxes of [28], which have been confirmed independently numerically [13].
} 
Here, $\mathcal{Y}$ is an arbitrary coefficient, and the Wronskian $W$ and $\mathcal{X}, \mathcal{Z}$ coefficients are given by

$$
\begin{aligned}
W & =2 i m \tilde{\Omega} \frac{\Gamma(2 h)}{\Gamma(h-i m-s)}, \\
\mathcal{X} & =R_{0} \mathcal{M}^{\mathrm{D} \prime}\left(R_{0}\right)\left(2 s a_{2}-a_{1}-2 a_{2}\right)+\mathcal{M}^{\mathrm{D}}\left(R_{0}\right)\left(a_{0}-2 s a_{1}-2 s a_{2}+4 s^{2} a_{2}+a_{2} V\left(R_{0}\right)\right), \\
\mathcal{Z} & =R_{0} \mathcal{W}^{\text {in' }}\left(R_{0}\right)\left(2 s a_{2}-a_{1}-2 a_{2}\right)+\mathcal{W}^{\text {in }}\left(R_{0}\right)\left(a_{0}-2 s a_{1}-2 s a_{2}+4 s^{2} a_{2}+a_{2} V\left(R_{0}\right)\right)
\end{aligned}
$$

where the potential is defined in (A.54) with $\Omega=m \tilde{\Omega}$ and $s=-2$ is understood.

If one attaches this solution to the asymptotically flat region, matching with 4.19 requires that the coefficient multiplying the homogenous solution be given by $\mathcal{Y}=R_{0}^{-2 s} W A$. One then finds

$$
B=\frac{\mathcal{Z}}{R_{0}^{4} W} \sqrt{2 \pi} \delta(\Omega-m \tilde{\Omega})
$$

where the delta function originates because only one frequency is present. The asymptotic behavior of the solution is then given by 4.23 where $B$ is now known. Note that the factors of $R_{0}$ cancel out in the final expression. Also note that

$$
\delta \psi_{4}=O\left(\lambda^{1 / 3}\right)
$$

The power of $1 / 3$ originates from the NHEK limit which was fine-tuned to include the ISCO in the NHEK region, see (2.7).

At $\hat{x} \rightarrow \infty$,

$$
\delta \psi_{4}(\hat{x} \rightarrow \infty) \rightarrow \frac{1}{2}\left(\ddot{h}_{+}-i \ddot{h}_{\times}\right)
$$

The energy and angular momentum flux in gravitational waves is given in terms of the Landau-Lifschitz pseudo-tensor as

$$
\begin{aligned}
\left(\frac{d E}{d \hat{t}}\right)^{\infty} & =\frac{1}{16 \pi} \int_{0}^{2 \pi} d \hat{\phi} \int_{0}^{\pi} \sin \theta d \theta \hat{r}^{2}\left\{\left(\dot{h}_{+}\right)^{2}+\left(\dot{h}_{\times}\right)^{2}\right\} \\
\left(\frac{d J}{d \hat{t}}\right)^{\infty} & =-\frac{1}{16 \pi} \int_{0}^{2 \pi} d \hat{\phi} \int_{0}^{\pi} \sin \theta d \theta \hat{r}^{2}\left\{\dot{h}_{+} \frac{\partial h_{+}}{\partial \hat{\phi}}+\dot{h}_{\times} \frac{\partial h_{\times}}{\partial \hat{\phi}}\right\} .
\end{aligned}
$$

where denotes a $\hat{t}$ derivative. Since $\frac{\partial h_{+}}{\partial \hat{\phi}}=-2 M \dot{h}_{+}$at leading order in $\lambda \rightarrow 0$, we have $\left(\frac{d J}{d \hat{t}}\right)^{\infty}=-2 M\left(\frac{d E}{d \hat{t}}\right)^{\infty}$.

In the case of a single mode $m$, the energy and angular momentum flux are given by

$$
\left(\frac{d E}{d \hat{t}}\right)^{\infty}=\int_{0}^{2 \pi} d \hat{\phi} \int_{0}^{\pi} \sin \theta d \theta \hat{r}^{2} \frac{\delta \psi_{4}^{*} \delta \psi_{4}}{4 \pi \hat{\omega}^{2}}=\frac{2 M^{8}}{m^{2}}|B \mathcal{K}|^{2}=O\left(\lambda^{\frac{4}{3} \operatorname{Re}(h)}\right)
$$


The energy flux is suppressed by the factor $\lambda^{\frac{4}{3} \operatorname{Re}(h)}$, as already noticed in $[13$. The dominant modes are the complex modes with $\operatorname{Re}(h)=\frac{1}{2}$. The formula (C.21) exactly matches with Eq. (77) of [13] after using the ISCO value $R_{0}=2^{1 / 3}$ where their $x_{0}=R_{0} \epsilon^{2 / 3}$ and $\epsilon$ is our $\lambda$. The flux of energy at the horizon is given by 62

$$
\left(\frac{d E}{d \hat{t}}\right)^{H}=\frac{128 \hat{\omega} \hat{k}\left(\hat{k}^{2}+4 \epsilon^{2}\right)\left(\hat{k}^{2}+16 \epsilon^{2}\right)\left(2 M r_{+}\right)^{5}}{|C|^{2}}\left|Z_{\text {hole }}^{2}\right|
$$

where $\epsilon=\frac{\lambda}{2 M}+o(\lambda)$ can be neglected compared to $\hat{k}=\hat{\omega}-\frac{m}{2 M}=O\left(\lambda^{2 / 3}\right)$. After matching the conventions of 62$]$ we find

$$
\begin{aligned}
Z_{\text {hole }} & =\lambda^{-\frac{4}{3}}\left(M^{2}\right) R_{0}^{2 s} \frac{\mathcal{X}+\mathcal{Y}}{W}(-2 i \Omega)^{i m+s} \\
|C|^{2} & =\left(m^{2}+(h-2)^{2}\right)\left(m^{2}+(h-1)^{2}\right)\left(m^{2}+h^{2}\right)\left(m^{2}+(h+1)^{2}\right) .
\end{aligned}
$$

We checked that the formula (C.22 with C.23 exactly matches with Eq. (76) of [13 after using the ISCO value $R_{0}=2^{1 / 3}$ and $\frac{R_{0}^{2 s}}{W} \mathcal{Y}=A$.

\section{Relevant integrals and QNM overtone summation}

In this appendix, we provide further details about several integrals which have been used in the main text and about the QNM overtone summations.

- Integrals involving an exponential of an exponential:

$$
\int_{-\infty}^{\infty} \mathrm{d} z e^{-p z} e^{-y e^{-z}}=y^{-p} \Gamma(p)
$$

This is 3.331 p. 340 of [48] for

$$
\operatorname{Re}(p)>0, \quad \operatorname{Re}(y)>0
$$

By analytic continuation, the first inequality relaxes to the condition that $p$ shouldn't be a non-positive integer.

- Integrals involving an exponential of a hyperbolic function:

$$
\int_{0}^{\infty} \mathrm{d} z \quad e^{-p z}\left(\sinh \frac{z}{2}\right)^{-2 h} e^{-y \operatorname{coth} \frac{z}{2}}=\left(\frac{y}{2}\right)^{-h} \Gamma(h+p) W_{-p,-h{ }_{+} \frac{1}{2}}(2 y) .
$$

The first equality is 3.547 (10) p. 386 of [48] up to a minus sign correction, which is emphasized here in the box. The conditions for (D.3) to hold are

$$
\operatorname{Re}(h+p)>0, \quad \operatorname{Re}(y)>0 .
$$


- Integrals involving arbitrary powers of hyperbolic functions:

$$
\begin{aligned}
\int_{0}^{\infty} & \mathrm{d} z \quad e^{-p z}\left(\operatorname{coth} \frac{z}{2}+\chi\right)^{-y}(\sinh z+\chi(\cosh z-1))^{-h} \\
& =(1+\chi)^{-y-h} 2^{h} B(h+p, 1-h+y)_{2} F_{1}\left(h+y, h+p, 1+y+p,-\frac{1-\chi}{1+\chi}\right)
\end{aligned}
$$

if

$$
\operatorname{Re}(h+p)>0, \quad \operatorname{Re}(1-h+y)>0, \quad \chi>-1 .
$$

$\underline{\text { Proof: }}$

$$
\begin{aligned}
\int_{0}^{\infty} & \mathrm{d} z \quad e^{-p z}\left(\operatorname{coth} \frac{z}{2}+\chi\right)^{-y}(\sinh z+\chi(\cosh \kappa t-1))^{-h} \\
& =2^{-h} \int_{0}^{\infty} \mathrm{d} z e^{-p z}\left(\operatorname{coth} \frac{z}{2}+\chi\right)^{-y-h}\left(\sinh \frac{z}{2}\right)^{-2 h} \\
& =2^{h} \int_{0}^{\infty} \mathrm{d} z e^{(-p-h) z}\left(1+\chi+(1-\chi) e^{-z}\right)^{-y-h}\left(1-e^{-z}\right)^{y-h}
\end{aligned}
$$

If $\chi>-1$, this can be written as

$$
\begin{aligned}
\int_{0}^{\infty} & \mathrm{d} z \quad e^{-p z}\left(\operatorname{coth} \frac{z}{2}+\chi\right)^{-y}(\sinh z+\chi(\cosh z-1))^{-h} \\
& =(1+\chi)^{-y-h} 2^{h} \int_{0}^{\infty} \mathrm{d} z e^{(-p-h) z}\left(1+\frac{(1-\chi)}{(1+\chi)} e^{-z}\right)^{-y-h}\left(1-e^{-z}\right)^{y-h} \\
& =(1+\chi)^{-y-h} 2^{h} B(h+p, 1-h+y) \\
& \times{ }_{2} F_{1}\left(h+y, h+p, 1+y+p,-\frac{1-\chi}{1+\chi}\right)
\end{aligned}
$$

using integral 3.312 (3) page 337 of [48] under the conditions

$$
\operatorname{Re}(h+p)>0, \quad \operatorname{Re}(1-h+y)>0, \quad\left|\arg \left(1+\frac{1-\chi}{1+\chi}\right)\right|<\pi
$$

where the last condition is satisfied in particular for $\chi>-1$.

Using the integral expression for a hypergeometric function in two variables $F_{2}$, 9.184 (2) on page 1030, along with 3.312 (3) page 337 of [48] one can also write

$$
\begin{aligned}
& \int_{0}^{\infty} \mathrm{d} z e^{(-p-h) z}\left(1+\chi+(1-\chi) e^{-z}\right)^{-y-h}\left(1-e^{-z}\right)^{y-h} \\
= & -\chi(1+y+h) B(h+p, 1-h+y) F_{2}(1+y+h ; p+h, 1 ; 1+y+p, 2 ;-(1-\chi),-\chi) \\
+ & B(h+p, 1-h+y)_{2} F_{1}(h+y, h+p, 1+y+p,-(1-\chi))
\end{aligned}
$$


if

$$
\operatorname{Re}(h+p)>0, \quad \operatorname{Re}(1+y-h)>0, \quad|\arg (2-\chi)|<\pi
$$

such that this should hold for $\chi<-1$.

- Integrals involving arbitrary powers of a polynomial:

$$
\begin{aligned}
& \int_{\tan \frac{\zeta}{2}}^{\infty} \mathrm{d} \bar{T} e^{-p \bar{T}}\left(\frac{\bar{T} \cos \zeta-\frac{1}{2}\left(1-\bar{T}^{2}\right) \sin \zeta}{\left(\cos \frac{\zeta}{2}+\sin \frac{\zeta}{2} \bar{T}\right)^{2}}\right)^{-y}\left(\bar{T} \cos \zeta-\frac{1}{2}\left(1-\bar{T}^{2}\right) \sin \zeta\right)^{-h} \\
= & \left(\tan \frac{\zeta}{2}\right)^{y} \Gamma(1-h-y) p^{h-1} e^{-\frac{p}{2}\left(\tan \frac{\zeta}{2}-\cot \frac{\zeta}{2}\right)} W_{y, \frac{1}{2}-h}\left(\frac{2 p}{\sin \zeta}\right)
\end{aligned}
$$

if $\sin \zeta>0$.

Proof:

$$
\begin{aligned}
& \int_{\tan \frac{\zeta}{2}}^{\infty} \mathrm{d} \bar{T} e^{-p \bar{T}}\left(\cos \frac{\zeta}{2}+\sin \frac{\zeta}{2} \bar{T}\right)^{2 y}\left(\bar{T} \cos \zeta-\frac{1}{2}\left(1-\bar{T}^{2}\right) \sin \zeta\right)^{-h-y} \\
= & \left(\frac{\sin \zeta}{2}\right)^{-y-h}\left(\sin \frac{\zeta}{2}\right)^{2 y} \int_{\tan \frac{\zeta}{2}}^{\infty} d \bar{T} e^{-p \bar{T}}\left(\bar{T}-\tan \frac{\zeta}{2}\right)^{-h-y}\left(\bar{T}+\cot \frac{\zeta}{2}\right)^{-h+y} \\
= & \left(\frac{\sin \zeta}{2}\right)^{-y-h}\left(\sin \frac{\zeta}{2}\right)^{2 y} e^{-p \tan \frac{\zeta}{2}} \int_{0}^{\infty} d x e^{-p x} x^{-h-y}\left(x+\frac{2}{\sin \zeta}\right)^{-h+y} \\
= & \left(\tan \frac{\zeta}{2}\right)^{y} \Gamma(1-h-y) p^{h-1} e^{-\frac{p}{2}\left(\tan \frac{\zeta}{2}-\cot \frac{\zeta}{2}\right)} W_{y, \frac{1}{2}-h}\left(\frac{2 p}{\sin \zeta}\right)
\end{aligned}
$$

In the last step we have used the integral representation of Whittaker function

$$
W_{k, m}(z)=\frac{e^{-z / 2} z^{k}}{\Gamma(1 / 2-k+m)} \int_{0}^{\infty} t^{-k-1 / 2+m}\left(1+\frac{t}{z}\right)^{k-1 / 2+m} e^{-t} d t
$$

and

$$
\operatorname{Re}(p)>0, \quad \operatorname{Re}(1-h-y)>0, \quad \sin \zeta>0 .
$$

- Integrals involving the exponential of an inverse:

$$
\int_{0}^{\infty} \mathrm{d} \bar{T} e^{-p \bar{T}} e^{\frac{-y}{T}} \bar{T}^{-2 h}=2\left(\frac{y}{p}\right)^{\frac{1}{2}-h} K_{1-2 h}(2 \sqrt{p y})
$$

which is valid for

$$
\operatorname{Re}(y)>0, \quad \operatorname{Re}(p)>0 .
$$

From 3.471(9) page 370 [48] for

$$
\operatorname{Re}\left(\alpha \pm \mu+\frac{3}{2}\right)>0, \quad \operatorname{Re}\left(s+\frac{q}{2}\right)>0, \quad q>0 .
$$


- Summation involving a hypergeometric function:

$$
\begin{aligned}
\sum_{N=0}^{\infty} & \frac{(-1)^{N} x^{N}}{N !} \frac{\Gamma\left(1-c_{+}\right)}{\Gamma\left(1-c_{+}-N\right)}{ }_{2} F_{1}\left(c_{-},-N, 1-c_{+}-N, z\right) \\
= & (1-z x)^{-c_{-}}(1-x)^{-c_{+}}
\end{aligned}
$$

Proof:

$$
\begin{aligned}
\sum_{N=0}^{\infty} & \frac{(-1)^{N} x^{N}}{N !} \frac{\Gamma\left(1-c_{+}\right)}{\Gamma\left(1-c_{+}-N\right)_{2}} F_{1}\left(c_{-},-N, 1-c_{+}-N, z\right) \\
= & \sum_{N=0}^{\infty} \frac{\Gamma\left(c_{+}+N\right)}{N ! \Gamma\left(c_{+}\right)}{ }_{2} F_{1}\left(c_{-},-N, 1-c_{+}-N, z\right) x^{N} \\
= & \sum_{N=0}^{\infty} \frac{\left(c_{+}\right)_{N}}{N !}{ }_{2} F_{1}\left(c_{-},-N, 1-c_{+}-N, z\right) x^{N} \\
= & \sum_{N=0}^{\infty} \sum_{k=0}^{N}(-1)^{k}\left(\begin{array}{c}
N \\
k
\end{array}\right) \frac{\left(c_{+}\right)_{N}\left(c_{-}\right)_{k}}{N !\left(1-c_{+}-N\right)_{k}} z^{k} x^{N} \\
= & \sum_{N=0}^{\infty} \sum_{k=0}^{N} \frac{\left(c_{-}\right)_{k}\left(c_{+}\right)_{N-k}}{k !(N-k) !}(z x)^{k}(x)^{N-k} \\
= & \sum_{N=0}^{\infty} \sum_{k=0}^{\infty} \frac{\left(c_{-}\right)_{k}\left(c_{+}\right)_{N}}{k ! N !}(z x)^{k} x^{N} \\
= & (1-z x)^{-c_{-}}(1-x)^{-c_{+}}
\end{aligned}
$$

where the last line uses

$$
(1+x)^{c_{+}}=\sum_{N=0}^{\infty}\left(-c_{+}\right)_{N} \frac{(-x)^{N}}{N !}
$$

- After using the property of the hypergeometric function [66, Eq. 15.8.1]

$$
{ }_{2} F_{1}(a, b, c ; z)=(1-z)^{-a}{ }_{2} F_{1}\left(a, c-b, c ; \frac{z}{z-1}\right)
$$

we also have

$$
\begin{aligned}
\sum_{N=0}^{\infty} & \frac{(-1)^{N} x^{N}}{N !} \frac{\Gamma\left(1-c_{+}\right)}{\Gamma\left(1-c_{+}-N\right)}(1-z)^{-c_{-}}{ }_{2} F_{1}\left(c_{-}, 1-c_{+}, 1-c_{+}-N, \frac{z}{z-1}\right) \\
= & (1-z x)^{-c_{-}}(1-x)^{-c_{+}}
\end{aligned}
$$

- Involving one Whittaker function:

$$
\sum_{N=0}^{\infty} \frac{(-a)^{N}}{N !} W_{h+N, h-\frac{1}{2}}(z)=z^{h} e^{-\frac{z}{2}}(1-a)^{-2 h} e^{-\frac{z a}{1-a}}
$$




\section{References}

[1] H. Audley et al., "Laser Interferometer Space Antenna," 1702.00786.

[2] P. Amaro-Seoane, J. R. Gair, M. Freitag, M. Coleman Miller, I. Mandel, C. J. Cutler, and S. Babak, "Astrophysics, detection and science applications of intermediate- and extreme mass-ratio inspirals," Class. Quant. Grav. 24 (2007) R113-R169, astro-ph/0703495.

[3] S. Babak, J. Gair, A. Sesana, E. Barausse, C. F. Sopuerta, C. P. L. Berry, E. Berti, P. Amaro-Seoane, A. Petiteau, and A. Klein, "Science with the space-based interferometer LISA. V: Extreme mass-ratio inspirals," Phys. Rev. D95 (2017), no. 10, 103012, 1703.09722.

[4] S. L. Detweiler, "Perspective on gravitational self-force analyses," Class. Quant. Grav. 22 (2005) S681-S716, gr-qc/0501004.

[5] L. Barack, "Gravitational self force in extreme mass-ratio inspirals," Class. Quant. Grav. 26 (2009) 213001, 0908.1664.

[6] L. Blanchet, A. Spallicci, and B. Whiting, "Mass and motion in general relativity. Proceedings, School on Mass, Orleans, France, June 23-25, 2008," Fundam. Theor. Phys. 162 (2011) pp.1-624.

[7] E. Poisson, A. Pound, and I. Vega, "The Motion of point particles in curved spacetime," Living Rev. Rel. 14 (2011) 7, 1102.0529.

[8] P. Amaro-Seoane, J. R. Gair, A. Pound, S. A. Hughes, and C. F. Sopuerta, "Research Update on Extreme-Mass-Ratio Inspirals," J. Phys. Conf. Ser. 610 (2015), no. 1, 012002, 1410.0958.

[9] B. Wardell and A. Gopakumar, "Self-force: Computational Strategies," Fund. Theor. Phys. 179 (2015) 487-522, 1501.07322.

[10] A. Pound, "Motion of small objects in curved spacetimes: An introduction to gravitational self-force," Fund. Theor. Phys. 179 (2015) 399-486, 1506.06245.

[11] S. E. Gralla, S. A. Hughes, and N. Warburton, "Inspiral into Gargantua," Class. Quant. Grav. 33 (2016), no. 15, 155002, 1603.01221.

[12] K. S. Thorne, The Science of Interstellar. New York: WW Norton \& Company, 2014. 
[13] S. E. Gralla, A. P. Porfyriadis, and N. Warburton, "Particle on the Innermost Stable Circular Orbit of a Rapidly Spinning Black Hole," Phys. Rev. D92 (2015), no. 6, 064029, 1506.08496 .

[14] G. Compère and R. Oliveri, "Self-similar accretion in thin discs around near-extremal black holes," Mon. Not. Roy. Astron. Soc. 468 (2017), no. 4, 4351-4361, 1703.00022.

[15] L. Brenneman, "Measuring Supermassive Black Hole Spins in Active Galactic Nuclei," 1309.6334.

[16] L. W. Brenneman and C. S. Reynolds, "Constraining Black Hole Spin Via X-ray Spectroscopy," Astrophys. J. 652 (2006) 1028-1043, astro-ph/0608502.

[17] A. C. Fabian, A. Zoghbi, R. R. Ross, P. Uttley, L. C. Gallo, W. N. Brandt, A. J. Blustin, T. Boller, M. D. Caballero-Garcia, J. Larsson, J. M. Miller, G. Miniutti, G. Ponti, R. C. Reis, C. S. Reynolds, Y. Tanaka, and A. J. Young, "Broad line emission from iron K- and L-shell transitions in the active galaxy 1H0707-495," Nature 459 (May, 2009) 540-542.

[18] K. S. Thorne, "Disk-Accretion onto a Black Hole. II. Evolution of the Hole," Astrophysical Journal 191 (July, 1974) 507-520.

[19] E. Berti and M. Volonteri, "Cosmological black hole spin evolution by mergers and accretion," Astrophys. J. 684 (2008) 822-828, 0802.0025.

[20] A. Sa̧dowski, M. Bursa, M. Abramowicz, W. Kluźniak, J.-P. Lasota, R. Moderski, and M. Safarzadeh, "Spinning up black holes with super-critical accretion flows," Astronomy 65 Astrophysics 532 (Aug., 2011) A41, 1102.2456.

[21] J. M. Bardeen and G. T. Horowitz, "The Extreme Kerr throat geometry: A Vacuum analog of AdS(2) x S**2," Phys. Rev. D60 (1999) 104030, hep-th/9905099.

[22] A. J. Amsel, G. T. Horowitz, D. Marolf, and M. M. Roberts, "No Dynamics in the Extremal Kerr Throat," JHEP 09 (2009) 044, 0906.2376.

[23] O. J. C. Dias, H. S. Reall, and J. E. Santos, "Kerr-CFT and gravitational perturbations," JHEP 08 (2009) 101, 0906.2380.

[24] I. Bredberg, T. Hartman, W. Song, and A. Strominger, "Black Hole Superradiance From Kerr/CFT," JHEP 04 (2010) 019, 0907.3477.

[25] M. Guica, T. Hartman, W. Song, and A. Strominger, "The Kerr/CFT Correspondence," Phys. Rev. D80 (2009) 124008, 0809.4266. 
[26] I. Bredberg, C. Keeler, V. Lysov, and A. Strominger, "Cargese Lectures on the Kerr/CFT Correspondence," Nucl. Phys. Proc. Suppl. 216 (2011) 194-210, 1103.2355 .

[27] G. Compère, "The Kerr/CFT correspondence and its extensions: a comprehensive review," Living Rev. Rel. 15 (2012) 11, 1203.3561.

[28] A. P. Porfyriadis and A. Strominger, "Gravity waves from the Kerr/CFT correspondence," Phys. Rev. D90 (2014), no. 4, 044038, 1401.3746.

[29] S. Hadar, A. P. Porfyriadis, and A. Strominger, "Gravity Waves from Extreme-Mass-Ratio Plunges into Kerr Black Holes," Phys. Rev. D90 (2014), no. 6, 064045, 1403.2797.

[30] S. Hadar, A. P. Porfyriadis, and A. Strominger, "Fast plunges into Kerr black holes," JHEP 07 (2015) 078, 1504.07650.

[31] S. Hadar and A. P. Porfyriadis, "Whirling orbits around twirling black holes from conformal symmetry," JHEP 03 (2017) 014, 1611.09834.

[32] S. Aretakis, "Decay of Axisymmetric Solutions of the Wave Equation on Extreme Kerr Backgrounds," J. Funct. Anal. 263 (2012) 2770-2831, 1110.2006.

[33] T. Piran, J. Shaham, and J. Katz, "High Efficiency of the Penrose Mechanism for Particle Collisions," Astrophysical Journal Letters 196 (Mar., 1975) L107.

[34] M. Banados, J. Silk, and S. M. West, "Kerr Black Holes as Particle Accelerators to Arbitrarily High Energy," Phys. Rev. Lett. 103 (2009) 111102, 0909.0169.

[35] S. E. Gralla, A. Zimmerman, and P. Zimmerman, "Transient Instability of Rapidly Rotating Black Holes," Phys. Rev. D94 (2016), no. 8, 084017, 1608.04739.

[36] H. Yang, F. Zhang, A. Zimmerman, D. A. Nichols, E. Berti, and Y. Chen, "Branching of quasinormal modes for nearly extremal Kerr black holes," Phys. Rev. D87 (2013), no. 4, 041502, 1212.3271.

[37] H. Yang, A. Zimmerman, A. Zenginoglu, F. Zhang, E. Berti, and Y. Chen, "Quasinormal modes of nearly extremal Kerr spacetimes: spectrum bifurcation and power-law ringdown," Phys. Rev. D88 (2013), no. 4, 044047, 1307.8086. [Phys. Rev.D88,044047(2013)].

[38] S. E. Gralla, A. Lupsasca, and A. Strominger, "Near-horizon Kerr Magnetosphere," Phys. Rev. D93 (2016), no. 10, 104041, 1602.01833. 
[39] S. E. Gralla and P. Zimmerman, "Critical Exponents of Extremal Kerr Perturbations," 1711.00855.

[40] R. P. Geroch, "Limits of spacetimes," Commun. Math. Phys. 13 (1969) 180-193.

[41] J. M. Bardeen, W. H. Press, and S. A. Teukolsky, "Rotating black holes: Locally nonrotating frames, energy extraction, and scalar synchrotron radiation," Astrophys. J. 178 (1972) 347.

[42] E. W. Leaver, "Spectral decomposition of the perturbation response of the schwarzschild geometry," Phys. Rev. D 34 (Jul, 1986) 384-408.

[43] S. Hod, "Slow relaxation of rapidly rotating black holes," Phys. Rev. D. 78 (Oct., 2008) 084035, 0811.3806 .

[44] G. B. Cook and M. Zalutskiy, "Gravitational perturbations of the Kerr geometry: High-accuracy study," Phys. Rev. D90 (2014), no. 12, 124021, 1410.7698.

[45] S. Hod, "On the branching of the quasinormal resonances of near-extremal Kerr black holes," Eur. Phys. J. C75 (2015), no. 11, 520, 1510.05604.

[46] A. Zimmerman, H. Yang, F. Zhang, D. A. Nichols, E. Berti, and Y. Chen, "Reply to "On the branching of quasinormal resonances of near-extremal Kerr black holes" by Shahar Hod," 1510.08159.

[47] L. M. Burko and G. Khanna, "Gravitational waves from a plunge into a nearly extremal Kerr black hole," Phys. Rev. D94 (2016), no. 8, 084049, 1608.02244.

[48] I. Gradshteyn and I. Ryzhik, Table of Integrals, Series, and Products Eighth Edition. Elsevier, 2015.

[49] F. Pretorius and D. Khurana, "Black hole mergers and unstable circular orbits," Class. Quant. Grav. 24 (2007) S83-S108, gr-qc/0702084.

[50] U. Sperhake, V. Cardoso, F. Pretorius, E. Berti, T. Hinderer, and N. Yunes, "Cross section, final spin and zoom-whirl behavior in high-energy black hole collisions," Phys. Rev. Lett. 103 (2009) 131102, 0907.1252.

[51] E. Berti, V. Cardoso, T. Hinderer, M. Lemos, F. Pretorius, U. Sperhake, and N. Yunes, "Semianalytical estimates of scattering thresholds and gravitational radiation in ultrarelativistic black hole encounters," Phys. Rev. D81 (2010) 104048, 1003.0812 .

[52] C. Gundlach, S. Akcay, L. Barack, and A. Nagar, "Critical phenomena at the threshold of immediate merger in binary black hole systems: the extreme mass ratio case," Phys. Rev. D86 (2012) 084022, 1207.5167. 
[53] M. A. Abramowicz and J. P. Lasota, "Spin-up of black holes by thick accretion disks," Acta Astron. 30 (1980) 35-39.

[54] M. Colleoni, L. Barack, A. G. Shah, and M. van de Meent, "Self-force as a cosmic censor in the Kerr overspinning problem," Phys. Rev. D92 (2015), no. 8, 084044, 1508.04031 .

[55] C. Merlin, A. Ori, L. Barack, A. Pound, and M. van de Meent, "Completion of metric reconstruction for a particle orbiting a Kerr black hole," Phys. Rev. D94 (2016), no. 10, 104066, 1609.01227.

[56] S. A. Teukolsky, "Perturbations of a Rotating Black Hole. I. Fundamental Equations for Gravitational, Electromagnetic, and Neutrino-Field Perturbations," Astrophysics Journal 185 (Oct., 1973) 635-648.

[57] S. Chandrasekhar, "The mathematical theory of black holes," in Oxford, UK: Clarendon (1992) 646 p., OXFORD, UK: CLARENDON (1985) 646 P. 1985.

[58] S. Chandrasekhar and K. S. Thorne, "The Mathematical Theory of Black Holes," American Journal of Physics 53 (Oct., 1985) 1013-1015.

[59] R. M. Wald, "On perturbations of a Kerr black hole.," Journal of Mathematical Physics 14 (1973) 1453-1461.

[60] J. M. Cohen and L. S. Kegeles, "Space-time perturbations," Physics Letters A 54 (Aug., 1975) 5-7.

[61] P. L. Chrzanowski, "Vector potential and metric perturbations of a rotating black hole," Phys. Rev. D. 11 (Apr., 1975) 2042-2062.

[62] S. A. Teukolsky and W. H. Press, "Perturbations of a rotating black hole. III Interaction of the hole with gravitational and electromagnetic radiation," Astrophys. J. 193 (Oct., 1974) 443-461.

[63] S. A. Hughes, "The Evolution of circular, nonequatorial orbits of Kerr black holes due to gravitational wave emission," Phys. Rev. D61 (2000), no. 8, 084004, gr-qc/9910091. [Erratum: Phys. Rev.D90,no.10,109904(2014)].

[64] N. Warburton. http://nielswarburton.net/code/gravitational-wave-flux-from-thenear-horizon-regime-of-a-near-extremal-kerr-black-hole/.

[65] A. G. Shah, J. L. Friedman, and T. S. Keidl, "EMRI corrections to the angular velocity and redshift factor of a mass in circular orbit about a Kerr black hole," Phys. Rev. D86 (2012) 084059, 1207.5595. 
[66] F. Olver, D. Lozier, B. R.F., and C. C.W., NIST Handbook of Mathematical Functions. NIST and Cambridge University Press, 2010. 